\title{
A Domino $N$-Amidoacylation/Aldol type Condensation Approach to the Synthesis of the Topo-I Inhibitor Rosettacin and Derivatives
}

By

Frédéric Pin, ${ }^{\dagger}$ Sébastien Comesse,$^{\dagger}$ Morgane Sanselme ${ }^{\ddagger}$ and Adam Dä̈ch ${ }^{\dagger *}$

+ URCOM, EA 3221, UFR des Sciences \& Techniques, Université du Havre, 25 rue Philippe Lebon, BP: 540, F-76058 Le Havre Cedex, France

Phone : (+033) 2-32-74-44-03 ; Fax: (+033) 2-32-74-43-91

E-mail: adam.daich@univ-lehavre.fr

* Sciences et Méthodes Séparatives, UPRES EA 3233 IRCOF - Université de ROUEN, 1 Rue Tesnière F76821 Mont-Saint-Aignan Cedex, France

\begin{abstract}
:
The pot, atom and step economic synthesis of Rosettacin topo-I poison and its derivatives has been achieved using a novel domino $\mathrm{N}$-amidoacylation/Aldol type condensation, followed by decarboxylation of the ester function. The key domino procedure simply involves mixing $\mathrm{HOBt}$ ester as new reagent, lactam and $\mathrm{NaH}$ together in THF or THF/ DMF. The reaction seems to be general and led to suitable $\mathrm{N}$-heterocyclic products in moderate to good yields.
\end{abstract}

\section{Graphical Abstract:}
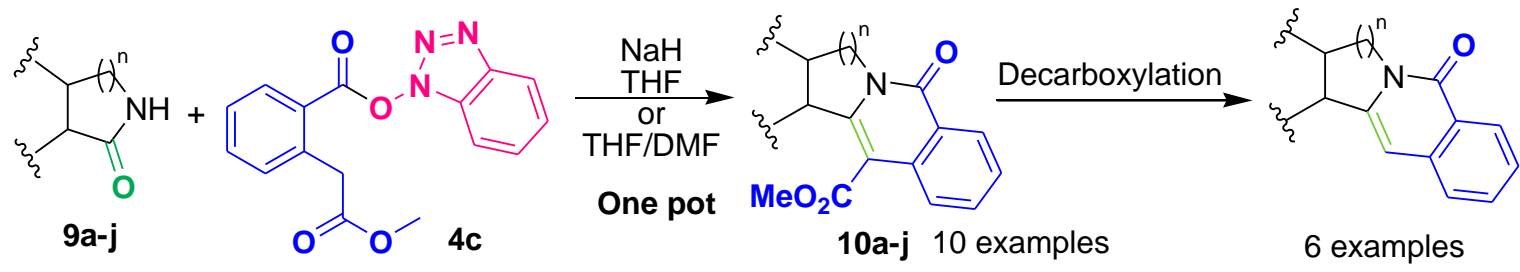

10a-j 10 examples

6 examples<smiles>O=c1c2ccccc2cc2n1Cc1cc3ccccc3nc1-2</smiles>

Rosettacin (2g)<smiles>O=c1c2ccccc2cc2n1Cc1nc3ccccc3cc1-2</smiles>

Isorosettacin (2h)<smiles>Cc1c2c(nc3ccccc13)-c1cc3ccccc3c(=O)n1C2</smiles>

13-Methylrosettacin (2k)<smiles>Cc1c2c(nc3ccccc13)Cn1c-2cc2ccccc2c1=O</smiles>

6-Methylisorosettacin (2i)<smiles>O=c1c2ccccc2cc2n1Cc1c-2nc2ccccc2c1-c1ccccc1</smiles>

13-Phenylrosettacin (2l)<smiles>O=c1c2ccccc2cc2n1Cc1nc3ccccc3c(-c3ccccc3)c1-2</smiles>

6-Phenylisorosettacin (2j) 


\section{Table of Cotents}

\section{Experimental part}

General remarks. $\quad 03$

Synthesis of the substrate 4c. 03

$N$-Benzotriazol-1-yl 2-methoxycarbonylmethylbenzoate (4c).

Transformation of $4 \mathrm{a}$ or $\mathbf{4 b}$ into products 7 and 8. Method A and Method B. 04

4-Methyl-3-( $N$-pyrrolidin-2-one)-1-oxo- $1 H$-isochromene-3,4-dicarboxylate (7). 04

6,12-Dimethyl 6,12-dihydrodibenzo[a,e]cyclooctene-5,11-dione-6,12-dicarboxylate (8). 04

Typical procedure for the domino N-amidoacylation/Aldol type condensation. 05

1,2-Dihydro-10-methoxycarbonylpyrrolo[1,2-b]isoquinolin-5(3H)-one (10a). 05

11-Methoxycarbonyl-1,2,3,4-tetrahydropiperidino[1,2-b]isoquinolin-6-one (10b). 05

12-Methoxycarbonyl-7H-isoindolo[2,1- $b$ ] isoquinolin-5-one (10c). 06

7-Benzyl-12-methoxycarbonylisoindolo[2,1-b]isoquinolin-5-one (10d). 06

7,8-Dihydro-14-methoxycarbonyl-13H-indolo[2',3':3,4]pyrido[1,2-b]isoquinolin-5-one (10e). 06

7,7-Dimethyl-14-methoxycarbonyl-7a,13-dihydrobenzo[e][1,3]thiazino[2',3':5,1]imidazo[1,2-b]isoquinolin-5-one (10f).

6-Methoxycarbonyl-12H-5,11a-diazadibenzo[b,h]fluoren-11-one (10g). 07

5-Methoxycarbonyl-12H-11,12a-diazadibenzo[ $b, h]$ fluoren-13-one (10h). 07

6-Methyl-5-methoxycarbonyl-12H-11,12a-diazadibenzo[b,h]fluoren-13-one (10i). 08

5-Methoxycarbonyl-6-phenyl-12H-11,12a-diazadibenzo[ $b, h]$ fluoren-13-one (10j). 08

Oxidation of 10a into keto-lactame 12. 08

10-Methoxycarbonyl-1,2,3,5-tetrahydropyrrolo[1,2-b]isoquinolin-1,5-dione (12). 09

General procedure for Friedländer synthesis of quinolines.

6-Methoxycarbonyl-12H-5,11a-diazadibenzo[b,h]fluoren-11-one (10g). 09

13-Methyl-6-methoxycarbonyl-12H-5,11a-diazadibenzo[b,h]fluoren-11-one (10k). 09

6-Methoxycarbonyl-13-phenyl-12H-5,11a-diazadibenzo[b,h]fluoren-11-one (101). 10

General protocol for the ester hydrolysis and ensuing decarboxylation 10g-l. 10

12H-5,11a-Diazadibenzo[b,h]fluoren-11-one (Rosettacin: 2g). 10

13-Methyl-12H-5,11a-diazadibenzo[b,h]fluoren-11-one (2k). 11

13-Phenyl-12 $H$-5,11a-diazadibenzo[b,h]fluoren-11-one (2l). 11

12H-11,12a-Diazadibenzo[b,h]fluoren-13-one (Isorosettacin: 2h). 11

6-Methyl-12H-11,12a-diazadibenzo[b,h]fluoren-13-one (2i). 12

6-Phenyl-12H-11,12a-diazadibenzo[ $b, h]$ fluoren-13-one (2j). 12

\section{List of ${ }^{1} \mathrm{H}$ and ${ }^{13} \mathrm{C}$ NMR spectra of all products described herein.}

$N$-Benzotriazol-1-yl 2-methoxycarbonylmethylbenzoate (4c).

$13-14$

4-Methyl-3-( $N$-pyrrolidin-2-one) 1-oxo- $1 H$-isochromene-3,4-dicarboxylate (7). 14-15

6,12-Dimethyl 6,12-dihydrodibenzo[a,e]cyclooctene-5,11-dione-6,12-dicarboxylate (8). $\quad$ 16-17

1,2-Dihydro-10-methoxycarbonylpyrrolo[1,2-b]isoquinolin-5(3H)-one (10a). 17-18

11-Methoxycarbonyl-1,2,3,4-tetrahydropiperidino[1,2-b]isoquinolin-6-one (10b). 19-20

12-Methoxycarbonyl-7 $\mathrm{H}$-isoindolo[2,1-b]isoquinolin-5-one (10c). 20-21

7-Benzyl-12-methoxycarbonylisoindolo[2,1-b]isoquinolin-5-one (10d). 22-23

7,8-Dihydro-14-methoxycarbonyl-13H-indolo[2',3':3,4]pyrido[1,2-b]isoquinolin-5-one (10e). 23-24 
7,7-Dimethyl-14-methoxycarbonyl-7a,13-dihydrobenzo[e][1,3]thiazino[2',3':5,1]imidazo[1,2- $b]$ isoquinolin-5-one (10f).

6-Methoxycarbonyl-12H-5,11a-diazadibenzo[b,h]fluoren-11-one (10g). 26-27

5-Methoxycarbonyl-12H-11,12a-diazadibenzo[b,h]fluoren-13-one (10h). 28-29

6-Methyl-5-methoxycarbonyl-12H-11,12a-diazadibenzo[b,h]fluoren-13-one (10i). 29-30

5-Methoxycarbonyl-6-phenyl-12H-11,12a-diazadibenzo[b,h]fluoren-13-one (10j). 31-32

13-Methyl-6-methoxycarbonyl-12H-5,11a-diazadibenzo[b,h]fluoren-11-one (10k). 32-33

6-Methoxycarbonyl-13-phenyl-12H-5,11a-diazadibenzo[ $b, h]$ fluoren-11-one (101). 34-35

10-Methoxycarbonyl-1,2,3,5-tetrahydropyrrolo[1,2-b]isoquinolin-1,5-dione (12). 35-36

12H-5,11a-Diazadibenzo[b,h]fluoren-11-one (Rosettacin: $\mathbf{2 g}$ ). 37-38

12H-11,12a-Diazadibenzo[b,h]fluoren-13-one (Isorosettacin: $\mathbf{2 h}$ ). 38-39

6-Methyl-12H-11,12a-diazadibenzo[b,h]fluoren-13-one (2i). 40-41

6-Phenyl-12H-11,12a-diazadibenzo[b,h]fluoren-13-one (2j). $\quad$ 41-42

13-Methyl-12H-5,11a-diazadibenzo[b,h]fluoren-11-one (2k). 43-44

13-Phenyl-12H-5,11a-diazadibenzo[b,h]fluoren-11-one (2l). 44-45

\section{General remarks.}

\section{Experimental part}

Melting points were taken with a capillary melting point apparatus and are uncorrected. The infrared (IR) absorption spectra were determined as solutions in potassium bromide and are indicated in $\mathrm{cm}^{-1}$. The ${ }^{1} \mathrm{H}$ and ${ }^{13} \mathrm{C}$ NMR spectra were recorded as solutions in $\mathrm{CDCl}_{3}$ or DMSO- $d_{6}$ at 200 or $300 \mathrm{MHz}\left({ }^{1} \mathrm{H}\right)$ and 50.3 or $75 \mathrm{MHz}\left({ }^{13} \mathrm{C}\right)$, respectively. Chemical shifts $(\delta)$ are expressed in ppm relative to TMS as internal standard. Thin layer chromatography (TLC) was performed using silica gel analytical plates $\left(F_{254}\right)$ of $0.25 \mathrm{~mm}$ thickness. The detection on TLC plates was performed by UV light at 254 or $365 \mathrm{~nm}$ or using iodine vapor. Mass spectra (MS) were measured on a mass spectrometer using electron impact ionization (EI, $70 \mathrm{eV}$ ). The analytical results of elemental analysis are within $0.4 \%$ of theoretical values and were obtained from INSA institution at Rouen, F-76130 Mt-St-Aignan in France. All solvents used herein were distilled according to the standard protocols.

- Synthesis of the substrate 4c: To a solution of $o$-methoxycarbonylmethylbenzoic acid $(6,3 \mathrm{~g}$, $15 \mathrm{mmol})$ in anhydrous THF $(65 \mathrm{~mL})$ was added 1-hydroxybenzotriazole monohydrate $(2.09$ $\mathrm{g}, 15 \mathrm{mmol})$ followed by $N, N^{\prime}$-dicyclohexylcarbodiimide $(1.18 \mathrm{~g}, 15 \mathrm{mmol})$ dissolved in 13 $\mathrm{mL}$ of THF and the reaction was stirred overnight at room temperature. The entire reaction was filtered through a column of Celite 545. The filtrate was concentrated under reduced pressure and the crude product was purified by flash chromatography on silica gel using cyclohexane/ethyl acetate $(7: 3)$ as eluent to give $4.70 \mathrm{~g}$ of the $\mathrm{HOBt}$ ester $4 \mathrm{c}$ in nearly quantitative yield.<smiles>CC(=O)Cc1ccccc1C(=O)On1nnc2ccccc21</smiles> 
$\mathrm{N}$-Benzotriazol-1-yl 2-methoxycarbonylmethylbenzoate $(\mathbf{4 c})$. This product was isolated as a white solid in $93 \%$ yield; $R_{f} 0.51$ (cyclohexane/AcOEt $1: 1$ ); mp $111^{\circ} \mathrm{C}$; IR $(\mathrm{KBr}) v 1788,1736$, 1446, 1438, 1378, 1348, 1232, 1172, 1092, $981 \mathrm{~cm}^{-1} ;{ }^{1} \mathrm{H}$ NMR $\left(200 \mathrm{MHz}, \mathrm{CDCl}_{3}\right) \delta 3.64(\mathrm{~s}, 3 \mathrm{H}$, $\left.\mathrm{CO}_{2} \mathrm{Me}\right), 4.06\left(\mathrm{~s}, 2 \mathrm{H}, \mathrm{CH}_{2}\right), 7.38-7.46\left(\mathrm{~m}, 2 \mathrm{H}, \mathrm{H}_{\text {aro }}\right), 7.49-7.58\left(\mathrm{~m}, 3 \mathrm{H}, \mathrm{H}_{\text {aro }}\right), 7.68$ (ddd, $1 \mathrm{H}, \mathrm{H}_{\text {aro }}, J$ $=7.8,7.0$ and $1.6 \mathrm{~Hz}), 8.06\left(\mathrm{~d}, 1 \mathrm{H}, \mathrm{H}_{\text {aro }}, J=8.6 \mathrm{~Hz}\right), 8.40\left(\mathrm{dd}, 1 \mathrm{H}, \mathrm{H}_{\text {aro }}, J=7.8\right.$ and $\left.1.6 \mathrm{~Hz}\right) ;{ }^{13} \mathrm{C}$ NMR (75 MHz, $\left.\mathrm{CDCl}_{3}\right) \delta 40.0\left(\mathrm{CH}_{2}\right), 52.4\left(\mathrm{CH}_{3}\right), 108.9\left(\mathrm{CH}_{\text {aro }}\right), 120.5\left(\mathrm{CH}_{\mathrm{aro}}\right), 124.9\left(\mathrm{C}_{\mathrm{q}}\right), 125.0$ $\left(\mathrm{CH}_{\text {aro }}\right), 128.2\left(\mathrm{CH}_{\text {aro }}\right), 128.8\left(\mathrm{CH}_{\text {aro }}\right), 129.0\left(\mathrm{C}_{\mathrm{q}}\right), 131.7\left(\mathrm{CH}_{\text {aro }}\right), 132.9\left(\mathrm{CH}_{\text {aro }}\right), 134.9\left(\mathrm{CH}_{\text {aro }}\right)$, $137.8\left(\mathrm{C}_{\mathrm{q}}\right), 143.7\left(\mathrm{C}_{\mathrm{q}}\right), 163.2(\mathrm{C}=\mathrm{O}), 171.4(\mathrm{C}=\mathrm{O})$. Anal. Calcd for $\mathrm{C}_{16} \mathrm{H}_{13} \mathrm{~N}_{3} \mathrm{O}_{4}(311.09)$ : $\mathrm{C} 61.73$, H 4.21, N 13.50. Found: C 61.58, H 4.04, N 13.23.

- Transformation of $4 a$ or $4 b$ into products 7 and 8. Method A: A solution containing the crude chloride derivative $\mathbf{4 a}$ or $\mathbf{4 b}(15 \mathrm{mmol})$, freshly prepared from the ester-acid $\mathbf{6}$, and the pyrrolidin-2-one $(9 \mathbf{a}, 0.90 \mathrm{~mL}, 12 \mathrm{mmol})$ in toluene $(120 \mathrm{~mL})$ was heated at reflux for 12 hours. The mixture was cooled, diluted with ethyl acetate and washed with a saturated solution of sodium hydrogenocarbonate. The organic layers regrouped were dried over anhydrous $\mathrm{MgSO}_{4}$ then concentrated under reduced pressure. The crude mixture containing products 7 and $\mathbf{8}$ in 1:8.5 ratio was then purified by flash chromatography on silica gel using a mixture of cyclohexane/ethyl acetate (3:7) as eluent and gave $\mathbf{7}$ and 8, separable, in a global 68\% yield. Method B: The same mixture, but in 1:13 ratio in favor of product $\mathbf{B}$, was also obtained in $70 \%$ yield when the reaction was conducted in the presence of 2.5 equiv. $\mathrm{NaH}$ in dry THF instead of toluene at reflux for $12 \mathrm{~h}$. Samples for elemental analysis and/or X-ray diffraction were obtained by recrystallization from ethanol and/or ethanol/acetone. The ${ }^{1} \mathrm{H}$ NMR spectra of products was obtained from samples heavily contaminated by solvent(s) used during the chromatography purification and/or the recrystallization.<smiles>COCc1c(C(=O)N2CCCC2=O)oc(=O)c2ccccc12</smiles>

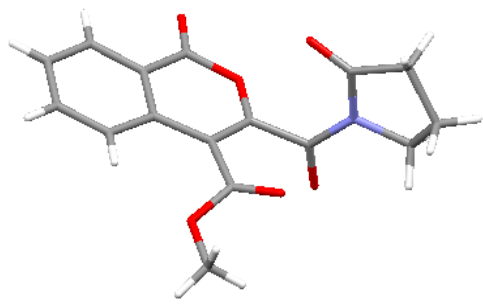

4-Methyl-3-( $N$-pyrrolidin-2-one) 1-oxo- $1 H$-isochromene-3,4-dicarboxylate (7). This product was isolated as a white solid in $8 \%$ yield $\left(30 \mathrm{mg}\right.$ ); $R_{f} 0.40$ (cyclohexane/AcOEt 3:7); $\mathrm{mp} 172^{\circ} \mathrm{C}$; IR (KBr) v 1755, 1695, 1624, 1485, 1455, 1438, 1360, 1254, 1229, 1188, 1158, 1130, 1056, 1017 $\mathrm{cm}^{-1} ;{ }^{1} \mathrm{H}$ NMR $\left(200 \mathrm{MHz}, \mathrm{CDCl}_{3}\right) \delta 2.18$ (quint, $2 \mathrm{H}, \mathrm{CH}_{2}, J=7.4 \mathrm{~Hz}$ ), $2.60\left(\mathrm{t}, 2 \mathrm{H}, \mathrm{CH}_{2} \mathrm{CO}, J=\right.$ $7.4 \mathrm{~Hz}), 3.85\left(\mathrm{~s}, 3 \mathrm{H}, \mathrm{CO}_{2} \mathrm{Me}\right), 3.92\left(\mathrm{t}, 2 \mathrm{H}, \mathrm{CH}_{2} \mathrm{~N}, J=7.4 \mathrm{~Hz}\right), 7.58\left(\mathrm{t}, 1 \mathrm{H}, \mathrm{H}_{\text {aro }}, J=7.8 \mathrm{~Hz}\right), 7.79$ $\left(\mathrm{t}, 1 \mathrm{H}, \mathrm{H}_{\mathrm{aro}}, J=7.8 \mathrm{~Hz}\right), 8.31\left(\mathrm{~d}, 2 \mathrm{H}, \mathrm{H}_{\mathrm{aro}}, J=8.6 \mathrm{~Hz}\right) ;{ }^{13} \mathrm{C} \mathrm{NMR}\left(50 \mathrm{MHz}, \mathrm{CDCl}_{3}\right) \delta 17.9\left(\mathrm{CH}_{2}\right)$, $32.5\left(\mathrm{CH}_{2}\right), 44.8\left(\mathrm{CH}_{2}\right), 52.9\left(\mathrm{CH}_{3}\right), 108.1\left(\mathrm{C}_{\mathrm{q}}\right), 120.3\left(\mathrm{C}_{\mathrm{q}}\right), 126.1\left(\mathrm{CH}_{\text {aro }}\right), 129.6\left(\mathrm{CH}_{\text {aro }}\right), 130.3$ $\left(\mathrm{CH}_{\text {aro }}\right), 133.4\left(\mathrm{C}_{\mathrm{q}}\right), 135.6\left(\mathrm{CH}_{\text {aro }}\right), 154.4\left(\mathrm{C}_{\mathrm{q}}\right), 159.7(\mathrm{C}=\mathrm{O}), 160.4(\mathrm{C}=\mathrm{O}), 164.4(\mathrm{C}=\mathrm{O}), 174.7$ $(\mathrm{C}=\mathrm{O})$. Anal. Calcd for $\mathrm{C}_{16} \mathrm{H}_{13} \mathrm{NO}_{6}$ (315.07): $\mathrm{C}$ 60.95, $\mathrm{H} 4.16, \mathrm{~N} 4.44$. Found: $\mathrm{C} 60.74, \mathrm{H} 4.07, \mathrm{~N}$ 4.29.

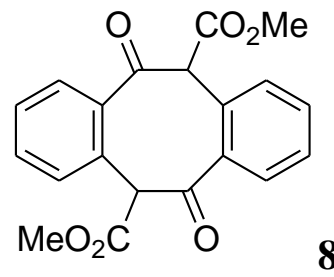


6,12-Dimethyl 6,12-dihydrodibenzo[a,e]cyclooctene-5,11-dione-6,12-dicarboxylate (8). This product was isolated as a yellow solid in $65 \%$ yield $(1.70 \mathrm{~g}) ; R_{f} 0.75$ (cyclohexane/AcOEt 1:1); mp $65^{\circ} \mathrm{C}$; IR $(\mathrm{KBr}) v 1740,1651,1609,1566,1485,1468,1436,1369,1314,1222,1174,1003 \mathrm{~cm}^{-1}$; ${ }^{1} \mathrm{H}$ NMR $\left(200 \mathrm{MHz}, \mathrm{CDCl}_{3}\right) \delta 3.89\left(\mathrm{~s}, 6 \mathrm{H}, \mathrm{CO}_{2} \mathrm{Me}\right), 5.53(\mathrm{~s}, 2 \mathrm{H}, \mathrm{CH}), 7.22-7.30\left(\mathrm{~m}, 4 \mathrm{H}, \mathrm{H}_{\text {aro }}\right)$, $7.58\left(\mathrm{dd}, 2 \mathrm{H}, \mathrm{H}_{\text {aro }}, J=7.8\right.$ and $\left.7.0 \mathrm{~Hz}\right), 8.14\left(\mathrm{~d}, 2 \mathrm{H}, \mathrm{H}_{\text {aro }}, J=7.8 \mathrm{~Hz}\right) ;{ }^{13} \mathrm{C} \mathrm{NMR}\left(75 \mathrm{MHz}, \mathrm{CDCl}_{3}\right)$ $\delta 56.1\left(2 \mathrm{CH}_{3}\right), 79.0(2 \mathrm{CH}), 117.4\left(2 \mathrm{C}_{\mathrm{q}}\right), 124.7\left(2 \mathrm{CH}_{\mathrm{aro}}\right), 125.6\left(2 \mathrm{CH}_{\mathrm{aro}}\right), 129.8\left(2 \mathrm{CH}_{\text {aro }}\right), 135.2$ $\left(2 \mathrm{CH}_{\text {aro }}\right), 140.0\left(2 \mathrm{C}_{\mathrm{q}}\right), 159.8(2 \mathrm{C}=\mathrm{O}), 161.3(2 \mathrm{C}=\mathrm{O})$. Anal. Calcd for $\mathrm{C}_{20} \mathrm{H}_{16} \mathrm{O}_{6}(352.09)$ : $\mathrm{C} 68.18$, H 4.58. Found: C 68.03, H 4.37.

- Typical procedure for the domino $N$-amidoacylation/Aldol type condensation. To a $0.1 \mathrm{M}$ THF solution (or $0.1 \mathrm{M}$ THF/DMF (1:1) for compounds $\mathbf{9 h}, \mathbf{9 i}$ and $\mathbf{9 j}$ ) of HOBt ester $\mathbf{4 c}$ (1 $\mathrm{mmol})$ and the appropriate lactam $(9,1 \mathrm{mmol})$ was added in one portion 2.5 equiv of $\mathrm{NaH}$. The mixture was then heated at reflux for 0.5 up to $12 \mathrm{~h}$ depending on the reagent (see Table 2). After cooling, the reaction was hydrolyzed with saturated $\mathrm{NH}_{4} \mathrm{Cl}$ solution and worked up by extracting either with ethyl acetate or with DCM. The organic layer was dried over anhydrous $\mathrm{MgSO}_{4}$ followed by concentration under reduced pressure. Pure product $\mathbf{1 0}$ was isolated ultimately by chromatography on silica gel column (cyclohexane/AcOEt as eluent). A sample for elemental analysis was obtained by washing the solid for three times with hot cyclohexane. The ${ }^{1} \mathrm{H}$ NMR spectra was obtained from sample heavily contaminated by mineral oil.

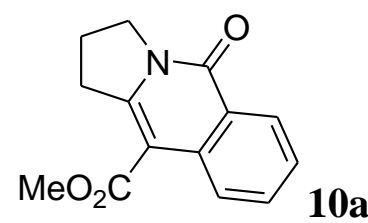

1,2-Dihydro-10-methoxycarbonylpyrrolo[1,2-b]isoquinolin-5(3H)-one (10a). This product was isolated as a white solid in $82 \%$ yield $\left(188 \mathrm{mg}\right.$ ); $R_{f} 0.22$ (cyclohexane/AcOEt $\left.1: 1\right) ; \mathrm{mp} 139^{\circ} \mathrm{C}$; IR $(\mathrm{KBr}) v 1708,1647,1483,1437,1398,1338,1295,1216,1116,1079 \mathrm{~cm}^{-1} ;{ }^{1} \mathrm{H}$ NMR $(200 \mathrm{MHz}$, $\left.\mathrm{CDCl}_{3}\right) \delta 2.18$ (quint, $2 \mathrm{H}, \mathrm{CH}_{2}, J=7.8 \mathrm{~Hz}$ ), $3.46\left(\mathrm{t}, 2 \mathrm{H}, \mathrm{CH}_{2}, J=7.8 \mathrm{~Hz}\right.$ ), $3.91\left(\mathrm{~s}, 3 \mathrm{H}, \mathrm{CO}_{2} \mathrm{Me}\right.$ ), $4.21\left(\mathrm{t}, 2 \mathrm{H}, \mathrm{CH}_{2} \mathrm{~N}, J=7.8 \mathrm{~Hz}\right.$ ), $7.44\left(\mathrm{t}, 1 \mathrm{H}, \mathrm{H}_{\text {aro }}, J=7.8 \mathrm{~Hz}\right.$ ), 7.66 (ddd, $1 \mathrm{H}, \mathrm{H}_{\text {aro }}, J=8.6,7.0$ and $1.6 \mathrm{~Hz}), 8.38-8.44\left(\mathrm{~m}, 2 \mathrm{H}, \mathrm{H}_{\mathrm{aro}}\right) ;{ }^{13} \mathrm{C} \mathrm{NMR}\left(50 \mathrm{MHz}, \mathrm{CDCl}_{3}\right) \delta 21.4\left(\mathrm{CH}_{2}\right), 34.3\left(\mathrm{CH}_{2}\right), 48.9$ $\left(\mathrm{CH}_{2}\right), 51.9\left(\mathrm{CH}_{3}\right), 104.2\left(\mathrm{C}_{\mathrm{q}}\right), 124.7\left(\mathrm{C}_{\mathrm{q}}\right), 125.2\left(\mathrm{CH}_{\text {aro }}\right), 126.5\left(\mathrm{CH}_{\text {aro }}\right), 127.6\left(\mathrm{CH}_{\text {aro }}\right), 132.9$ $\left(\mathrm{CH}_{\text {aro }}\right), 135.5\left(\mathrm{C}_{\mathrm{q}}\right), 151.5\left(\mathrm{C}_{\mathrm{q}}\right), 161.5(\mathrm{C}=\mathrm{O}), 167.1(\mathrm{C}=\mathrm{O})$. Anal. Calcd for $\mathrm{C}_{14} \mathrm{H}_{13} \mathrm{NO}_{3}(243.09)$ : C 69.12, H 5.39, N 5.76. Found: C 68.94, H 5.26, N 5.59.<smiles>CC(=O)c1c2n(c(=O)c3ccccc13)CCCC2</smiles>

11-Methoxycarbonyl-1,2,3,4-tetrahydropiperidino[1,2-b]isoquinolin-6-one (10b). This product was isolated as a white solid in $50 \%$ yield $\left(128 \mathrm{mg}\right.$ ); $R_{f} 0.46$ (cyclohexane/AcOEt $\left.1: 1\right) ; \mathrm{mp} 88^{\circ} \mathrm{C}$ (recrystallized from ethanol); IR (KBr) $v$ 2954, 1718, 1643, 1613, 1595, 1557, 1491, 1436, 1398, 1331, 1312, 1290, 1214, 1167, 1123, 1099, 1077, $1025 \mathrm{~cm}^{-1} ;{ }^{1} \mathrm{H}$ NMR $\left(200 \mathrm{MHz}, \mathrm{CDCl}_{3}\right) \delta 1.79$ $2.01\left(\mathrm{~m}, 4 \mathrm{H}, 2 \mathrm{CH}_{2}\right), 2.92\left(\mathrm{t}, 2 \mathrm{H}, \mathrm{CH}_{2}-\mathrm{C}, J=6.3 \mathrm{~Hz}\right), 3.95\left(\mathrm{~s}, 3 \mathrm{H}, \mathrm{CO}_{2} \mathrm{Me}\right), 4.15\left(\mathrm{t}, 2 \mathrm{H}, \mathrm{CH}_{2} \mathrm{~N}, J=\right.$ $6.3 \mathrm{~Hz}), 7.39-7.47\left(\mathrm{~m}, 1 \mathrm{H}, \mathrm{H}_{\text {aro }}\right), 7.56-7.67\left(\mathrm{~m}, 2 \mathrm{H}, \mathrm{H}_{\text {aro }}\right), 8.40\left(\mathrm{~d}, 1 \mathrm{H}, \mathrm{H}_{\text {aro }}, J=7.8 \mathrm{~Hz}\right) ;{ }^{13} \mathrm{C} \mathrm{NMR}$ $\left(75 \mathrm{MHz}, \mathrm{CDCl}_{3}\right) \delta 18.7\left(\mathrm{CH}_{2}\right), 21.8\left(\mathrm{CH}_{2}\right), 26.8\left(\mathrm{CH}_{2}\right), 41.3\left(\mathrm{CH}_{2}\right), 52.4\left(\mathrm{CH}_{3}\right), 109.2\left(\mathrm{C}_{\mathrm{q}}\right), 123.6$ $\left(\mathrm{CH}_{\text {aro }}\right), 123.8\left(\mathrm{C}_{\mathrm{q}}\right), 126.5\left(\mathrm{CH}_{\text {aro }}\right), 128.1\left(\mathrm{CH}_{\text {aro }}\right), 132.7\left(\mathrm{CH}_{\text {aro }}\right), 133.8\left(\mathrm{C}_{\mathrm{q}}\right), 143.0\left(\mathrm{C}_{\mathrm{q}}\right), 162.3$ $(\mathrm{C}=\mathrm{O}), 168.2(\mathrm{C}=\mathrm{O})$. Anal. Calcd for $\mathrm{C}_{15} \mathrm{H}_{15} \mathrm{NO}_{3}$ (257.11): C 70.02, H 5.88, N 5.44. Found: $\mathrm{C}$ 69.89, H 5.69, N 5.32. 
<smiles>COC(=O)c1c2n(c(=O)c3ccccc13)-c1ccccc1-2</smiles>

12-Methoxycarbonyl-7H-isoindolo[2,1-b]isoquinolin-5-one (10c). This product was isolated as a white solid in $72 \%$ yield $(209 \mathrm{mg}) ; R_{f} 0.39$ (cyclohexane/AcOEt $\left.1: 1\right) ; \mathrm{mp} 199^{\circ} \mathrm{C}$; IR $(\mathrm{KBr}) v$ 1723, 1650, 1620, 1608, 1485, 1472, 1436, 1384, 1313, 1241, 1208, 1156, $1024 \mathrm{~cm}^{-1}$; ${ }^{1} \mathrm{H}$ NMR $\left(200 \mathrm{MHz}, \mathrm{CDCl}_{3}\right.$, the ${ }^{1} \mathrm{H} \mathrm{NMR}$ spectra contains traces of water) $\delta 4.10\left(\mathrm{~s}, 3 \mathrm{H}, \mathrm{CO}_{2} \mathrm{Me}\right), 5.20(\mathrm{~s}$, $\left.2 \mathrm{H}, \mathrm{CH}_{2} \mathrm{~N}\right), 7.42-7.61\left(\mathrm{~m}, 4 \mathrm{H}, \mathrm{H}_{\mathrm{aro}}\right), 7.66-7.78\left(\mathrm{~m}, 3 \mathrm{H}, \mathrm{H}_{\mathrm{aro}}\right), 8.51\left(\mathrm{~d}, 1 \mathrm{H}, \mathrm{H}_{\text {aro }}, J=8.6 \mathrm{~Hz}\right) ;{ }^{13} \mathrm{C}$ NMR $\left(50 \mathrm{MHz}, \mathrm{CDCl}_{3}\right) \delta 52.1\left(\mathrm{CH}_{2}\right), 52.8\left(\mathrm{CH}_{3}\right), 106.1\left(\mathrm{C}_{\mathrm{q}}\right), 123.5\left(\mathrm{CH}_{\text {aro }}\right), 124.0\left(\mathrm{CH}_{\text {aro }}\right.$ and $\left.\mathrm{C}_{\mathrm{q}}\right)$, $124.4\left(\mathrm{CH}_{\text {aro }}\right), 127.1\left(\mathrm{CH}_{\text {aro }}\right), 127.7\left(\mathrm{CH}_{\text {aro }}\right), 128.6\left(\mathrm{CH}_{\text {aro }}\right), 130.8\left(\mathrm{CH}_{\text {aro }}\right), 132.7\left(\mathrm{C}_{\mathrm{q}}\right), 132.9$ $\left(\mathrm{CH}_{\text {aro }}\right), 135.1\left(\mathrm{C}_{\mathrm{q}}\right), 138.8\left(\mathrm{C}_{\mathrm{q}}\right), 141.5\left(\mathrm{C}_{\mathrm{q}}\right), 160.7(\mathrm{C}=\mathrm{O}), 167.7(\mathrm{C}=\mathrm{O})$. Anal. Calcd for $\mathrm{C}_{18} \mathrm{H}_{13} \mathrm{NO}_{3}$ (291.09): C 74.22, H 4.50, N 4.81. Found: C 74.09, H 4.38, N 4.67.

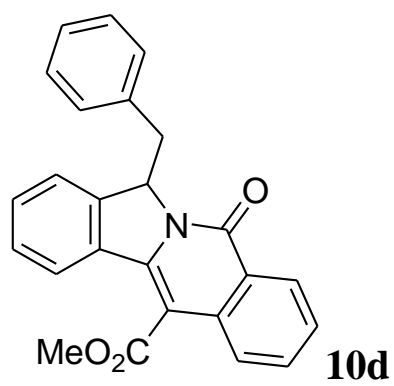

7-Benzyl-12-methoxycarbonylisoindolo[2,1-b]isoquinolin-5-one (10d). This product was isolated as a pink solid in $37 \%$ yield $(141 \mathrm{mg}) ; R_{f} 0.45$ (cyclohexane/AcOEt 7:3); mp $146^{\circ} \mathrm{C}$ (recrystallized from ethanol/acetone); IR (KBr) v 1723, 1653, 1619, 1607, 1484, 1436, 1353, 1316, 1297, 1241, 1217, 1199, 1167, $1148 \mathrm{~cm}^{-1}$; ${ }^{1} \mathrm{H}$ NMR $\left(300 \mathrm{MHz}, \mathrm{CDCl}_{3}\right.$, the ${ }^{1} \mathrm{H}$ NMR spectra contains traces of water and acetone) $\delta 3.18\left(\mathrm{dd}, 1 \mathrm{H}, \mathrm{CH}_{2}, J=13.2\right.$ and $\left.8.2 \mathrm{~Hz}\right), 3.95(\mathrm{dd}, 1 \mathrm{H}$, $\mathrm{CH}_{2}, J=13.2$ and $\left.3.0 \mathrm{~Hz}\right), 4.04\left(\mathrm{~s}, 3 \mathrm{H}, \mathrm{CO}_{2} \mathrm{Me}\right), 5.83(\mathrm{dd}, 1 \mathrm{H}, \mathrm{CHN}, J=8.2$ and $3.0 \mathrm{~Hz}), 6.96-$ $7.02\left(\mathrm{~m}, 3 \mathrm{H}, \mathrm{H}_{\mathrm{aro}}\right), 7.10-7.14\left(\mathrm{~m}, 3 \mathrm{H}, \mathrm{H}_{\mathrm{aro}}\right), 7.33-7.40\left(\mathrm{~m}, 2 \mathrm{H}, \mathrm{H}_{\mathrm{aro}}\right), 7.51-7.60\left(\mathrm{~m}, 2 \mathrm{H}, \mathrm{H}_{\mathrm{aro}}\right)$, 7.64$7.73\left(\mathrm{~m}, 2 \mathrm{H}, \mathrm{H}_{\text {aro }}\right), 8.53\left(\mathrm{~d}, 1 \mathrm{H}, \mathrm{H}_{\text {aro }}, J=7.9 \mathrm{~Hz}\right) ;{ }^{13} \mathrm{C} \mathrm{NMR}\left(75 \mathrm{MHz}, \mathrm{CDCl}_{3}\right) \delta 37.6\left(\mathrm{CH}_{2}\right), 52.8$ $\left(\mathrm{CH}_{3}\right), 64.8(\mathrm{CH}), 105.9\left(\mathrm{C}_{\mathrm{q}}\right), 123.7\left(\mathrm{CH}_{\text {aro }}\right), 123.8\left(\mathrm{CH}_{\text {aro }}\right), 124.4\left(\mathrm{CH}_{\text {aro }}\right), 124.8\left(\mathrm{C}_{\mathrm{q}}\right), 127.0$ $\left(\mathrm{CH}_{\text {aro }}\right), 127.2\left(\mathrm{CH}_{\text {aro }}\right), 127.8\left(\mathrm{CH}_{\text {aro }}\right), 128.4\left(2 \mathrm{CH}_{\text {aro }}\right), 128.8\left(\mathrm{CH}_{\text {aro }}\right), 130.0\left(2 \mathrm{CH}_{\text {aro }}\right), 130.3\left(\mathrm{CH}_{\text {aro }}\right)$, $132.0\left(\mathrm{C}_{\mathrm{q}}\right), 133.0\left(\mathrm{CH}_{\text {aro }}\right), 135.0\left(\mathrm{C}_{\mathrm{q}}\right), 135.8\left(\mathrm{C}_{\mathrm{q}}\right), 141.1\left(\mathrm{C}_{\mathrm{q}}\right), 142.7\left(\mathrm{C}_{\mathrm{q}}\right), 161.0(\mathrm{C}=\mathrm{O}), 167.8$ $(\mathrm{C}=\mathrm{O})$. Anal. Calcd for $\mathrm{C}_{25} \mathrm{H}_{19} \mathrm{NO}_{3}$ (381.14): C 78.72, H 5.02, N 3.67. Found: C 78.55, H 4.87, N 3.48 .<smiles></smiles>

7,8-Dihydro-14-methoxycarbonyl-13H-indolo[2',3' :3,4]pyrido[1,2-b]isoquinolin-5-one (10e). This product was isolated as a yellow solid in $30 \%$ yield $\left(103 \mathrm{mg}\right.$ ); $R_{f} 0.60$ (cyclohexane/AcOEt $1: 1)$; $\mathrm{mp} 238^{\circ} \mathrm{C}$; IR (KBr) v 3420, 1710, 1646, 1607, 1598, 1562, 1489, 1436, 1397, 1367, 1341, $1313,1290,1242,1224,1195,1165,1132,1110,1034,979 \mathrm{~cm}^{-1} ;{ }^{1} \mathrm{H}$ NMR $\left(200 \mathrm{MHz}, \mathrm{CDCl}_{3}\right.$, the ${ }^{1} \mathrm{H}$ NMR spectra contains traces of water and AcOEt) $\delta 3.09\left(\mathrm{t}, 2 \mathrm{H}, \mathrm{CH}_{2}, J=6.3 \mathrm{~Hz}\right), 4.04(\mathrm{~s}, 3 \mathrm{H}$, $\left.\mathrm{CO}_{2} \mathrm{Me}\right), 4.53\left(\mathrm{t}, 2 \mathrm{H}, \mathrm{CH}_{2} \mathrm{~N}, J=6.3 \mathrm{~Hz}\right), 7.16\left(\mathrm{dd}, 1 \mathrm{H}, \mathrm{H}_{\text {aro }}, J=7.8\right.$ and $\left.7.0 \mathrm{~Hz}\right), 7.24-7.32(\mathrm{~m}$, $\left.1 \mathrm{H}, \mathrm{H}_{\text {aro }}\right), 7.40\left(\mathrm{~d}, 2 \mathrm{H}, \mathrm{H}_{\text {aro }}, J=7.8 \mathrm{~Hz}\right), 7.48\left(\mathrm{~d}, 1 \mathrm{H}, \mathrm{H}_{\text {aro }}, J=7.8 \mathrm{~Hz}\right), 7.58-7.69\left(\mathrm{~m}, 2 \mathrm{H}, \mathrm{H}_{\text {aro }}\right)$, 
$8.45\left(\mathrm{~d}, 1 \mathrm{H}, \mathrm{H}_{\text {aro }}, J=8.6 \mathrm{~Hz}\right), 9.18(\mathrm{br} \mathrm{s}, 1 \mathrm{H}, \mathrm{NH}) ;{ }^{13} \mathrm{C} \mathrm{NMR}\left(50 \mathrm{MHz}, \mathrm{CDCl}_{3}\right) \delta 20.0\left(\mathrm{CH}_{2}\right), 41.6$ $\left(\mathrm{CH}_{2}\right), 53.5\left(\mathrm{CH}_{3}\right), 107.1\left(\mathrm{C}_{\mathrm{q}}\right), 112.2\left(\mathrm{CH}_{\text {aro }}\right), 118.1\left(\mathrm{C}_{\mathrm{q}}\right), 119.6\left(\mathrm{CH}_{\text {aro }}\right), 120.9\left(\mathrm{CH}_{\text {aro }}\right), 124.0$ $\left(\mathrm{CH}_{\text {aro }}\right), 124.6\left(\mathrm{C}_{\mathrm{q}}\right), 125.2\left(\mathrm{CH}_{\text {aro }}\right), 125.4\left(\mathrm{C}_{\mathrm{q}}\right), 126.9\left(\mathrm{C}_{\mathrm{q}}\right), 127.2\left(\mathrm{CH}_{\text {aro }}\right), 128.6\left(\mathrm{CH}_{\text {aro }}\right), 131.8\left(\mathrm{C}_{\mathrm{q}}\right)$, $133.0\left(\mathrm{CH}_{\text {aro }}\right), 133.9\left(\mathrm{C}_{\mathrm{q}}\right), 138.0\left(\mathrm{C}_{\mathrm{q}}\right), 162.0(\mathrm{C}=\mathrm{O}), 171.3(\mathrm{C}=\mathrm{O})$. Anal. Calcd for $\mathrm{C}_{21} \mathrm{H}_{16} \mathrm{~N}_{2} \mathrm{O}_{3}$ (344.12): C 73.24, H 4.68, N 8.13. Found: C 73.08, H 4.54, N 8.05.

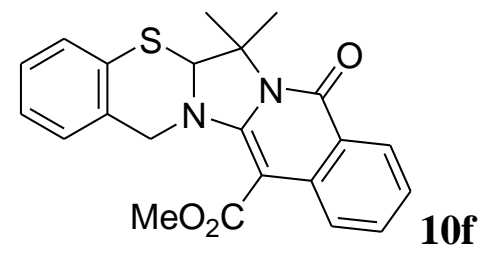

7,7-Dimethyl-14-methoxycarbonyl-7a,13-dihydrobenzo $[e][1,3]$ thiazino[2',3' $: 5,1]$ imidazo[1,2b]isoquinolin-5-one (10f). This product was isolated as a white solid in $47 \%$ yield $(184 \mathrm{mg}) ; R_{f}$ 0.40 (cyclohexane/AcOEt 4:1); mp $132^{\circ} \mathrm{C}$ (recrystallized from ethanol); IR (KBr) $v 1706,1657$, $1608,1554,1485,1450,1435,1401,1280,1240,1211,1135,1119,1031 \mathrm{~cm}^{-1} ;{ }^{1} \mathrm{H}$ NMR $(300$ $\left.\mathrm{MHz}, \mathrm{CDCl}_{3}\right) \delta 1.74\left(\mathrm{~s}, 3 \mathrm{H}, \mathrm{CH}_{3}\right), 2.00\left(\mathrm{~s}, 3 \mathrm{H}, \mathrm{CH}_{3}\right), 3.95\left(\mathrm{~s}, 3 \mathrm{H}, \mathrm{CO}_{2} \mathrm{Me}\right), 4.23\left(\mathrm{~d}, 1 \mathrm{H}, \mathrm{CH}_{2}, J=\right.$ $15.1 \mathrm{~Hz}), 4.71\left(\mathrm{~d}, 1 \mathrm{H}, \mathrm{CH}_{2}, J=15.1 \mathrm{~Hz}\right), 4.81(\mathrm{~s}, 1 \mathrm{H}, \mathrm{CH}), 7.19-7.32\left(\mathrm{~m}, 5 \mathrm{H}, \mathrm{H}_{\text {aro }}\right), 7.50-7.56(\mathrm{~m}$, $\left.1 \mathrm{H}, \mathrm{H}_{\text {aro }}\right), 7.77\left(\mathrm{~d}, 1 \mathrm{H}, \mathrm{H}_{\text {aro }}, J=8.3 \mathrm{~Hz}\right), 8.23\left(\mathrm{dd}, 1 \mathrm{H}, \mathrm{H}_{\text {aro }}, J=8.1\right.$ and $\left.1.2 \mathrm{~Hz}\right) ;{ }^{13} \mathrm{C} \mathrm{NMR}(75$ $\left.\mathrm{MHz}, \mathrm{CDCl}_{3}\right) \delta 22.7\left(\mathrm{CH}_{3}\right), 25.3\left(\mathrm{CH}_{3}\right), 51.0\left(\mathrm{CH}_{2}\right), 52.1\left(\mathrm{CH}_{3}\right), 64.6\left(\mathrm{C}_{\mathrm{q}}\right), 76.5(\mathrm{CH}), 88.9\left(\mathrm{C}_{\mathrm{q}}\right)$, $122.0\left(\mathrm{C}_{\mathrm{q}}\right), 122.4\left(\mathrm{CH}_{\text {aro }}\right), 124.0\left(\mathrm{CH}_{\text {aro }}\right), 126.7\left(\mathrm{CH}_{\text {aro }}\right), 127.6\left(\mathrm{CH}_{\text {aro }}\right), 128.1\left(\mathrm{CH}_{\text {aro }}\right), 128.5$ $\left(\mathrm{CH}_{\text {aro }}\right), 130.0\left(\mathrm{CH}_{\text {aro }}\right), 132.2\left(\mathrm{C}_{\mathrm{q}}\right), 133.1\left(\mathrm{CH}_{\text {aro }}\right), 134.0\left(\mathrm{C}_{\mathrm{q}}\right), 136.6\left(\mathrm{C}_{\mathrm{q}}\right), 147.5\left(\mathrm{C}_{\mathrm{q}}\right), 161.2(\mathrm{C}=\mathrm{O})$, 167.6 (C=O). Anal. Calcd for $\mathrm{C}_{22} \mathrm{H}_{20} \mathrm{~N}_{2} \mathrm{O}_{3} \mathrm{~S}$ (392.12): C 67.33, H 5.14, N 7.14. Found: $\mathrm{C} 67.21, \mathrm{H}$ $5.00, \mathrm{~N} 7.03$.

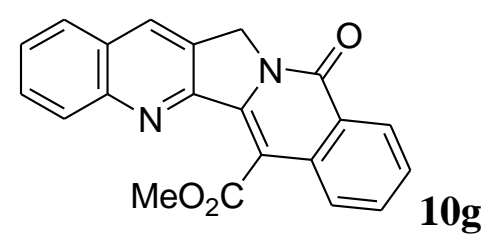

6-Methoxycarbonyl-12H-5,11a-diazadibenzo[b,h]fluoren-11-one $(\mathbf{1 0 g})$. This product was isolated as a yellow solid in $50 \%$ yield $\left(171 \mathrm{mg}\right.$ ); $R_{f} 0.37$ (cyclohexane/AcOEt $2: 3$ ); $\mathrm{mp} 260^{\circ} \mathrm{C}$ (recrystallized from ethanol); IR (KBr) $v$ 1726, 1664, 1633, 1602, 1470, 1377, 1333, 1297, 1241 $\mathrm{cm}^{-1} ;{ }^{1} \mathrm{H}$ NMR $(200 \mathrm{MHz}, \mathrm{DMSO}) \delta 4.16\left(\mathrm{~s}, 3 \mathrm{H}, \mathrm{CO}_{2} \mathrm{Me}\right), 5.38\left(\mathrm{~s}, 2 \mathrm{H}, \mathrm{CH}_{2} \mathrm{~N}\right), 7.68-7.96(\mathrm{~m}, 5 \mathrm{H}$, $\left.\mathrm{H}_{\text {aro }}\right)$, 8.09-8.46 (m, $\left.2 \mathrm{H}, \mathrm{H}_{\text {aro }}\right), 8.44\left(\mathrm{~d}, 1 \mathrm{H}, \mathrm{H}_{\text {aro }}, J=7.9 \mathrm{~Hz}\right), 8.68\left(\mathrm{~s}, 1 \mathrm{H}, \mathrm{H}_{\text {aro }}\right) ;{ }^{13} \mathrm{C} \mathrm{NMR}(75$ MHz, DMSO) $\delta 49.8\left(\mathrm{CH}_{2}\right), 52.5\left(\mathrm{CH}_{3}\right), 106.8\left(\mathrm{C}_{\mathrm{q}}\right), 124.3\left(\mathrm{CH}_{\text {aro }}\right), 124.8\left(\mathrm{C}_{\mathrm{q}}\right), 127.2\left(\mathrm{CH}_{\mathrm{aro}}\right)$, $127.7\left(\mathrm{CH}_{\text {aro }}\right.$ and $\left.\mathrm{C}_{\mathrm{q}}\right), 127.8\left(\mathrm{CH}_{\text {aro }}\right), 128.4\left(\mathrm{CH}_{\text {aro }}\right), 129.3\left(\mathrm{CH}_{\text {aro }}\right), 129.9\left(\mathrm{C}_{\mathrm{q}}\right), 130.3\left(\mathrm{CH}_{\text {aro }}\right), 131.2$ $\left(\mathrm{CH}_{\text {aro }}\right)$, $133.2\left(\mathrm{CH}_{\text {aro }}\right), 133.9\left(\mathrm{C}_{\mathrm{q}}\right), 138.2\left(\mathrm{C}_{\mathrm{q}}\right), 147.7\left(\mathrm{C}_{\mathrm{q}}\right), 152.1\left(\mathrm{C}_{\mathrm{q}}\right), 159.2(\mathrm{C}=\mathrm{O}), 166.4(\mathrm{C}=\mathrm{O})$. Anal. Calcd for $\mathrm{C}_{21} \mathrm{H}_{14} \mathrm{~N}_{2} \mathrm{O}_{3}$ (342.10): C 73.68, H 4.12, N 8.18. Found: C 73.50, H 4.03, N 8.04.<smiles>COC(=O)c1c2n(c(=O)c3ccccc13)Cc1nc3ccccc3cc1-2</smiles>

5-Methoxycarbonyl-12H-11,12a-diazadibenzo[b,h]fluoren-13-one (10h). This product was isolated as a brown solid in $47 \%$ yield $\left(160 \mathrm{mg}\right.$ ); $R_{f} 0.37$ (cyclohexane/AcOEt $\left.1: 1\right)$; mp $258^{\circ} \mathrm{C}$ (decomp); IR (KBr) $v$ 1724, 1654, 1608, 1482, 1376, 1339, 1290, 1243, 1204, 1156, $1119 \mathrm{~cm}^{-1}$; ${ }^{1} \mathrm{H}$ NMR (200 MHz, $\left.\mathrm{CDCl}_{3}\right) \delta 4.17\left(\mathrm{~s}, 3 \mathrm{H}, \mathrm{CO}_{2} \mathrm{Me}\right), 5.30\left(\mathrm{~s}, 2 \mathrm{H}, \mathrm{CH}_{2} \mathrm{~N}\right), 7.50-7.60\left(\mathrm{~m}, 2 \mathrm{H}, \mathrm{H}_{\mathrm{aro}}\right)$, $7.67-7.82\left(\mathrm{~m}, 3 \mathrm{H}, \mathrm{H}_{\mathrm{aro}}\right), 7.90\left(\mathrm{~d}, 1 \mathrm{H}, \mathrm{H}_{\text {aro }}, J=7.0 \mathrm{~Hz}\right), 8.09\left(\mathrm{~d}, 1 \mathrm{H}, \mathrm{H}_{\text {aro }}, J=8.6 \mathrm{~Hz}\right), 8.50(\mathrm{~d}, 1 \mathrm{H}$, $\left.\mathrm{H}_{\text {aro }}, J=7.8 \mathrm{~Hz}\right), 8.60\left(\mathrm{~s}, 1 \mathrm{H}, \mathrm{H}_{\text {aro }}\right) ;{ }^{13} \mathrm{C} \mathrm{NMR}\left(75 \mathrm{MHz}, \mathrm{CDCl}_{3}\right) \delta 52.4\left(\mathrm{CH}_{2}\right), 53.0\left(\mathrm{CH}_{3}\right), 107.7$ 
$\left(\mathrm{C}_{\mathrm{q}}\right), 124.7\left(\mathrm{C}_{\mathrm{q}}\right), 124.8\left(\mathrm{C}_{\mathrm{q}}\right), 125.0\left(\mathrm{CH}_{\text {aro }}\right), 127.3\left(\mathrm{C}_{\mathrm{q}}\right), 127.4\left(\mathrm{CH}_{\text {aro }}\right), 127.8\left(\mathrm{CH}_{\text {aro }}\right), 127.9\left(\mathrm{CH}_{\text {aro }}\right)$, $129.1\left(\mathrm{CH}_{\text {aro }}\right), 129.5\left(\mathrm{CH}_{\text {aro }}\right), 131.8\left(\mathrm{CH}_{\text {aro }}\right), 133.1\left(\mathrm{CH}_{\text {aro }}\right), 133.2\left(\mathrm{CH}_{\text {aro }}\right), 134.7\left(\mathrm{C}_{\mathrm{q}}\right), 140.1\left(\mathrm{C}_{\mathrm{q}}\right)$, $148.9\left(\mathrm{C}_{\mathrm{q}}\right), 158.8\left(\mathrm{C}_{\mathrm{q}}\right), 160.4(\mathrm{C}=\mathrm{O}), 167.3(\mathrm{C}=\mathrm{O})$. Anal. Calcd for $\mathrm{C}_{21} \mathrm{H}_{14} \mathrm{~N}_{2} \mathrm{O}_{3}(342.10)$ : $\mathrm{C} 73.68$, H 4.12, N 8.18. Found: C 73.57, H 3.97, N 7.89.<smiles>COc1c2c(nc3ccccc13)Cn1c-2c(C(=O)O)c2ccccc2c1=O</smiles>

6-Methyl-5-methoxycarbonyl-12H-11,12a-diazadibenzo $[\boldsymbol{b}, \boldsymbol{h}]$ fluoren-13-one $\quad$ (10i). This product was isolated as a yellow solid in $44 \%$ yield $(156 \mathrm{mg}) ; R_{f} 0.35$ (cyclohexane/AcOEt 1:1); $\mathrm{mp} 230^{\circ} \mathrm{C}$; IR (KBr) v 1721, 1651, 1592, 1479, 1443, 1435, 1371, 1337, 1301, 1240, 1209, 1173, $1161 \mathrm{~cm}^{-1}$; ${ }^{1} \mathrm{H}$ NMR $\left(200 \mathrm{MHz}, \mathrm{CDCl}_{3}\right.$, the ${ }^{1} \mathrm{H}$ NMR spectra contains traces of water) $\delta 2.81(\mathrm{~s}$, $\left.3 \mathrm{H}, \mathrm{CH}_{3}\right), 4.02\left(\mathrm{~s}, 3 \mathrm{H}, \mathrm{CO}_{2} \mathrm{Me}\right), 5.31\left(\mathrm{~s}, 2 \mathrm{H}, \mathrm{CH}_{2} \mathrm{~N}\right), 7.53-7.66\left(\mathrm{~m}, 2 \mathrm{H}, \mathrm{H}_{\text {aro }}\right), 7.70-7.81(\mathrm{~m}, 2 \mathrm{H}$, $\left.\mathrm{H}_{\text {aro }}\right), 8.07-8.15\left(\mathrm{~m}, 3 \mathrm{H}, \mathrm{H}_{\mathrm{aro}}\right), 8.52\left(\mathrm{dd}, 1 \mathrm{H}, \mathrm{H}_{\text {aro }}, J=7.8\right.$ and $\left.1.6 \mathrm{~Hz}\right) ;{ }^{13} \mathrm{C} \mathrm{NMR}(50 \mathrm{MHz}$, $\left.\mathrm{CDCl}_{3}\right) \delta 17.8\left(\mathrm{CH}_{3}\right), 53.0\left(\mathrm{CH}_{3}\right.$ and $\left.\mathrm{CH}_{2}\right), 109.3\left(\mathrm{C}_{\mathrm{q}}\right), 124.5\left(\mathrm{C}_{\mathrm{q}}\right), 124.6\left(\mathrm{C}_{\mathrm{q}}\right), 124.9\left(\mathrm{CH}_{\text {aro }}\right), 125.0$ $\left(\mathrm{CH}_{\text {aro }}\right), 127.2\left(\mathrm{CH}_{\text {aro }}\right), 127.8\left(2 \mathrm{CH}_{\text {aro }}\right), 128.0\left(\mathrm{C}_{\mathrm{q}}\right), 129.4\left(\mathrm{CH}_{\text {aro }}\right), 131.3\left(\mathrm{CH}_{\text {aro }}\right), 133.3\left(\mathrm{CH}_{\text {aro }}\right)$, 134.8 $\left(\mathrm{C}_{\mathrm{q}}\right), 142.4\left(\mathrm{C}_{\mathrm{q}}\right), 143.6\left(\mathrm{C}_{\mathrm{q}}\right), 148.0\left(\mathrm{C}_{\mathrm{q}}\right), 158.4\left(\mathrm{C}_{\mathrm{q}}\right), 160.5(\mathrm{C}=\mathrm{O}), 168.2(\mathrm{C}=\mathrm{O})$. Anal. Calcd for $\mathrm{C}_{22} \mathrm{H}_{16} \mathrm{~N}_{2} \mathrm{O}_{3}$ (356.12): C 74.15, H 4.53, N 7.86. Found: C 74.04, H 4.44, N 7.69.

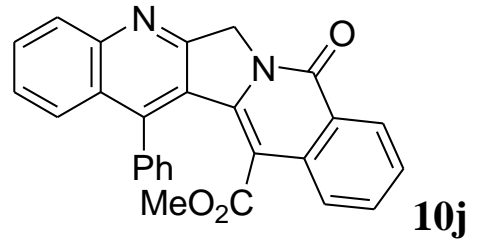

5-Methoxycarbonyl-6-phenyl-12H-11,12a-diazadibenzo $[\boldsymbol{b}, \boldsymbol{h}]$ fluoren-13-one $\quad(\mathbf{1 0 j}) . \quad$ This product was isolated as a yellow solid in $34 \%$ yield $\left(142 \mathrm{mg}\right.$ ); $R_{f} 0.38$ (cyclohexane/AcOEt 1:1); $\operatorname{mp~} 275^{\circ} \mathrm{C}$; IR (KBr) $v 1728,1649,1590,1478,1445,1371,1339,1306,1276,1239,1217,1176$, $1158 \mathrm{~cm}^{-1}$; ${ }^{1} \mathrm{H}$ NMR $\left(200 \mathrm{MHz}, \mathrm{CDCl}_{3}\right.$, the ${ }^{1} \mathrm{H}$ NMR spectra contains traces of water and AcOEt) $\delta 3.40\left(\mathrm{~s}, 3 \mathrm{H}, \mathrm{CO}_{2} \mathrm{Me}\right), 5.42\left(\mathrm{~s}, 2 \mathrm{H}, \mathrm{CH}_{2} \mathrm{~N}\right), 7.42-7.67\left(\mathrm{~m}, 8 \mathrm{H}, \mathrm{H}_{\text {aro }}\right), 7.74-7.81\left(\mathrm{~m}, 3 \mathrm{H}, \mathrm{H}_{\mathrm{aro}}\right), 8.15$ $\left(\mathrm{d}, 1 \mathrm{H}, \mathrm{H}_{\mathrm{aro}}, J=8.6 \mathrm{~Hz}\right), 8.52\left(\mathrm{~d}, 1 \mathrm{H}, \mathrm{H}_{\mathrm{aro}}, J=7.8 \mathrm{~Hz}\right) ;{ }^{13} \mathrm{C} \mathrm{NMR}\left(75 \mathrm{MHz}, \mathrm{CDCl}_{3}\right) \delta 52.5\left(\mathrm{CH}_{3}\right)$, $53.0\left(\mathrm{CH}_{2}\right), 109.7\left(\mathrm{C}_{\mathrm{q}}\right), 122.9\left(\mathrm{C}_{\mathrm{q}}\right), 124.6\left(\mathrm{C}_{\mathrm{q}}\right), 124.9\left(\mathrm{CH}_{\text {aro }}\right), 127.1\left(\mathrm{CH}_{\text {aro }}\right), 127.2\left(\mathrm{C}_{\mathrm{q}}\right), 127.3$ $\left(\mathrm{CH}_{\text {aro }}\right), 127.7\left(\mathrm{CH}_{\text {aro }}\right), 127.8\left(\mathrm{CH}_{\text {aro }}\right), 129.2\left(2 \mathrm{CH}_{\text {aro }}\right), 129.4\left(\mathrm{CH}_{\text {aro }}\right), 129.6\left(\mathrm{CH}_{\text {aro }}\right), 131.0\left(2 \mathrm{CH}_{\text {aro }}\right)$, $131.3\left(\mathrm{CH}_{\text {aro }}\right), 133.2\left(\mathrm{CH}_{\text {aro }}\right), 134.8\left(\mathrm{C}_{\mathrm{q}}\right), 135.0\left(\mathrm{C}_{\mathrm{q}}\right), 141.0\left(\mathrm{C}_{\mathrm{q}}\right), 146.9\left(\mathrm{C}_{\mathrm{q}}\right), 149.0\left(\mathrm{C}_{\mathrm{q}}\right), 159.0\left(\mathrm{C}_{\mathrm{q}}\right)$, $160.6(\mathrm{C}=\mathrm{O}), 165.8(\mathrm{C}=\mathrm{O})$. Anal. Calcd for $\mathrm{C}_{27} \mathrm{H}_{18} \mathrm{~N}_{2} \mathrm{O}_{3}(418.13)$ : C 77.50, H 4.34, N 6.69. Found: C 77.32, H 4.19, N 6.51.

- Oxidation of 10a into keto-lactame 12. A stirred mixture of tricyclic lactam 10a $(500 \mathrm{mg}, 2.1$ mmol) and Bredereck's reagent $(\mathbf{1 1}, 600 \mu \mathrm{L}, 2.9 \mathrm{mmol})$ were heated at $110{ }^{\circ} \mathrm{C}$ for $2 \mathrm{~h}$. After cooling at room temperature, the mixture was diluted with $15 \mathrm{~mL}$ of $\mathrm{H}_{2} \mathrm{O} / \mathrm{THF}$ (1:1). When a homogenous solution was obtained, sodium metaperiodate $(1.32 \mathrm{~g}, 6.2 \mathrm{mmol})$ was added and the mixture was stirred for an additional $0.5 \mathrm{~h}$. The solution was filtered through short column of Celite 545, washed with ethyl acetate and the filtrate was diluted by ethyl acetate. The organic layers combined were dried over anhydrous $\mathrm{MgSO}_{4}$ then evaporated under reduced pressure. The residue was purified by flash chromatography on silica gel column using cyclohexane/ethyl acetate $(1: 1)$ as the eluent and gave the expected product, namely 10methoxycarbonyl-1,2,3,5-tetrahydropyrrolo[1,2-b]isoquinolin-1,5-dione (12), in 60\% yield for two steps calculated from the tricyclic lactam 10a. Whatever the recrystallization (twice), this product decomposes slowly due probably to the presence of an impurity not yet determined. 
Only few amounts of product were isolated in pure form and used further for elemental analysis.

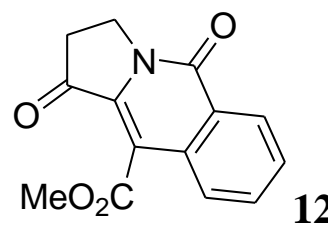

10-Methoxycarbonyl-1,2,3,5-tetrahydropyrrolo[1,2-b]isoquinolin-1,5-dione (12). This product was isolated as a yellow solid in $60 \%$ yield in two steps $\left(317 \mathrm{mg}\right.$ ); $R_{f} 0.22$ (cyclohexane/AcOEt 1:1); $\mathrm{mp} 131^{\circ} \mathrm{C}$; IR (KBr) v 1740, 1655, 1620, 1606, 1483, 1436, 1399, 1336, 1293, 1256, 1223, $1179,1161,1136,1103,1055 \mathrm{~cm}^{-1} ;{ }^{1} \mathrm{H}$ NMR $\left(200 \mathrm{MHz}, \mathrm{CDCl}_{3}\right) \delta 2.94\left(\mathrm{t}, 2 \mathrm{H}, \mathrm{CH}_{2}, J=7.0 \mathrm{~Hz}\right)$, $4.00\left(\mathrm{~s}, 3 \mathrm{H}, \mathrm{CO}_{2} \mathrm{Me}\right), 4.35\left(\mathrm{t}, 2 \mathrm{H}, \mathrm{CH}_{2} \mathrm{~N}, J=7.0 \mathrm{~Hz}\right), 7.61-7.78\left(\mathrm{~m}, 3 \mathrm{H}, \mathrm{H}_{\text {aro }}\right), 8.47\left(\mathrm{~d}, 1 \mathrm{H}, \mathrm{H}_{\text {aro }}, J\right.$ $=7.0 \mathrm{~Hz}) ;{ }^{13} \mathrm{C}$ NMR $\left(50 \mathrm{MHz}, \mathrm{CDCl}_{3}\right) \delta 34.1\left(\mathrm{CH}_{2}\right), 41.8\left(\mathrm{CH}_{2}\right), 53.4\left(\mathrm{CH}_{3}\right), 111.4\left(\mathrm{C}_{\mathrm{q}}\right), 126.4$ $\left(\mathrm{CH}_{\text {aro }}\right), 127.8\left(\mathrm{C}_{\mathrm{q}}\right), 128.1\left(\mathrm{CH}_{\text {aro }}\right), 130.0\left(\mathrm{CH}_{\text {aro }}\right), 132.1\left(\mathrm{C}_{\mathrm{q}}\right), 133.2\left(\mathrm{C}_{\mathrm{q}}\right), 133.4\left(\mathrm{CH}_{\mathrm{aro}}\right), 160.7$ $(\mathrm{C}=\mathrm{O}), 165.8(\mathrm{C}=\mathrm{O}), 196.4(\mathrm{C}=\mathrm{O})$. Anal. Calcd for $\mathrm{C}_{14} \mathrm{H}_{11} \mathrm{NO}_{4}(257.07)$ : $\mathrm{C}$ 65.37, H 4.31, N 5.44. Found: C 65.20, H 4.18, N 5.26.

- General procedure for Friedländer synthesis of quinolines. A solution of keto-lactame 12 (270 $\mathrm{mg}, 1 \mathrm{mmol})$, aromatic aminoketone (2-aminobenzaldehyde (13g), 2'-aminoacetophenone (13k) or 2-aminobenzophenone (13i), $1.1 \mathrm{mmol}$ ), $10 \mathrm{~mol} \%$ of PTSA (19 $\mathrm{mg}, 0.1$ mmol) in anhydrous toluene was heated in the Dean-Stark apparatus under stirring at reflux for $12 \mathrm{~h}$. After cooling at room temerature, the solvent was removed under reduced pressure and the residue was diluted with dichloromethane. The organic layers combined were washed twice with saturated solution of sodium hydrogenocarbonate, dried over anhydrous $\mathrm{MgSO}_{4}$ and then concentrated under reduced pressure. Pure products $10 \mathrm{~g}$ and $10 \mathrm{k}, \mathbf{l}$ were isolated ultimately by chromatography on silica gel column using cyclohexane/AcOEt as eluent.<smiles>COC(=O)c1c2n(c(=O)c3ccccc13)Cc1cc3ccccc3nc1-2</smiles>

6-Methoxycarbonyl-12H-5,11a-diazadibenzo[b,h]fluoren-11-one $(\mathbf{1 0 g})$. This product was isolated in $51 \%$ yield $(174 \mathrm{mg})$ and its characteristics are identical to those described above from the tandem $\mathrm{N}$-amidoacylation/Knovenagel olefination.<smiles>COC(=O)c1c2n(c(=O)c3ccccc13)Cc1c-2nc2ccccc2c1C</smiles>

13-Methyl-6-methoxycarbonyl-12H-5,11a-diazadibenzo $[\boldsymbol{b}, \boldsymbol{h}]$ fluoren-11-one $\quad(10 \mathrm{k}) . \quad$ This product was isolated as a brown solid in $71 \%$ yield $\left(253 \mathrm{mg}\right.$ ); $R_{f} 0.35$ (cyclohexane/AcOEt 1:1); $\mathrm{mp} 298^{\circ} \mathrm{C}$; IR (KBr) v 1726, 1661, 1625, 1605, 1339, 1297, 1282, $1215 \mathrm{~cm}^{-1}$; ${ }^{1} \mathrm{H}$ NMR $(200 \mathrm{MHz}$, $\mathrm{CDCl}_{3}$, the ${ }^{1} \mathrm{H}$ NMR spectra contains traces of water) $\delta 2.75\left(\mathrm{~s}, 3 \mathrm{H}, \mathrm{CH}_{3}\right), 4.19\left(\mathrm{~s}, 3 \mathrm{H}, \mathrm{CO}_{2} \mathrm{Me}\right)$, $5.27\left(\mathrm{~s}, 2 \mathrm{H}, \mathrm{CH}_{2} \mathrm{~N}\right), 7.55-7.63\left(\mathrm{~m}, 2 \mathrm{H}, \mathrm{H}_{\text {aro }}\right), 7.70-7.78\left(\mathrm{~m}, 3 \mathrm{H}, \mathrm{H}_{\text {aro }}\right), 8.02\left(\mathrm{~d}, 1 \mathrm{H}, \mathrm{H}_{\text {aro }}, J=8.6\right.$ $\mathrm{Hz}), 8.10\left(\mathrm{~d}, 1 \mathrm{H}, \mathrm{H}_{\text {aro }}, J=8.6 \mathrm{~Hz}\right), 8.54\left(\mathrm{~d}, 1 \mathrm{H}, \mathrm{H}_{\text {aro }}, J=7.8 \mathrm{~Hz}\right) ;{ }^{13} \mathrm{C} \mathrm{NMR}\left(75 \mathrm{MHz}, \mathrm{CDCl}_{3}\right) \delta$ $15.4\left(\mathrm{CH}_{3}\right), 49.5\left(\mathrm{CH}_{2}\right), 52.9\left(\mathrm{CH}_{3}\right), 108.3\left(\mathrm{C}_{\mathrm{q}}\right), 123.7\left(\mathrm{CH}_{\text {aro }}\right), 124.9\left(\mathrm{CH}_{\text {aro }}\right), 125.5\left(\mathrm{C}_{\mathrm{q}}\right), 127.6$ 
$\left(\mathrm{CH}_{\text {aro }}\right), 127.9\left(\mathrm{CH}_{\text {aro }}\right.$ and $\left.2 \mathrm{C}_{\mathrm{q}}\right), 128.0\left(\mathrm{CH}_{\text {aro }}\right), 129.8\left(\mathrm{CH}_{\text {aro }}\right), 131.0\left(\mathrm{CH}_{\text {aro }}\right), 133.2\left(\mathrm{CH}_{\text {aro }}\right), 134.8$ $\left(\mathrm{C}_{\mathrm{q}}\right), 139.0\left(\mathrm{C}_{\mathrm{q}}\right), 139.3\left(\mathrm{C}_{\mathrm{q}}\right), 149.0\left(\mathrm{C}_{\mathrm{q}}\right), 151.7\left(\mathrm{C}_{\mathrm{q}}\right), 160.7(\mathrm{C}=\mathrm{O}), 167.4(\mathrm{C}=\mathrm{O})$. Anal. Calcd for $\mathrm{C}_{22} \mathrm{H}_{16} \mathrm{~N}_{2} \mathrm{O}_{3}$ (356.12): C 74.15, H 4.53, N 7.86. Found: C 73.97, H 4.43, N 7.58.

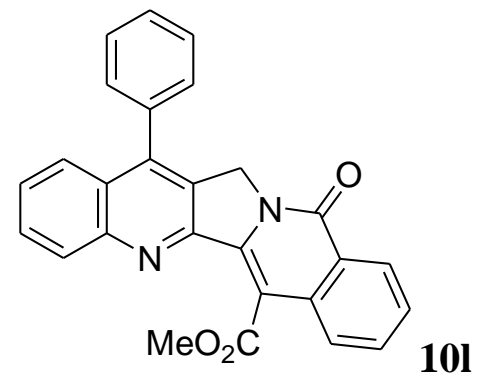

6-Methoxycarbonyl-13-phenyl-12H-5,11a-diazadibenzo $[\boldsymbol{b}, \boldsymbol{h}]$ fluoren-11-one (101). This product was isolated as a yellow solid in $86 \%$ yield $\left(357 \mathrm{mg}\right.$ ); $R_{f} 0.41$ (cyclohexane/AcOEt 7:3); $\mathrm{mp} 254^{\circ} \mathrm{C}$ (recrystallized from ethanol and washed with $\left.\mathrm{Et}_{2} \mathrm{O}\right)$; IR $(\mathrm{KBr}) v 1727,1662,1626,1605,1482$, 1393, 1342, 1284, 1246, $1222 \mathrm{~cm}^{-1}$; ${ }^{1} \mathrm{H}$ NMR (200 MHz, $\mathrm{CDCl}_{3}$, the ${ }^{1} \mathrm{H}$ NMR spectra contains traces of water and $\left.\mathrm{Et}_{2} \mathrm{O}\right) \delta 4.23\left(\mathrm{~s}, 3 \mathrm{H}, \mathrm{CO}_{2} \mathrm{Me}\right), 5.18\left(\mathrm{~s}, 2 \mathrm{H}, \mathrm{CH}_{2} \mathrm{~N}\right), 7.42-7.62\left(\mathrm{~m}, 7 \mathrm{H}, \mathrm{H}_{\mathrm{aro}}\right)$, $7.72-7.83\left(\mathrm{~m}, 4 \mathrm{H}, \mathrm{H}_{\text {aro }}\right), 8.17\left(\mathrm{~d}, 1 \mathrm{H}, \mathrm{H}_{\text {aro }}, J=7.8 \mathrm{~Hz}\right), 8.52\left(\mathrm{~d}, 1 \mathrm{H}, \mathrm{H}_{\text {aro }}, J=7.8 \mathrm{~Hz}\right) ;{ }^{13} \mathrm{C}$ NMR $(50$ $\left.\mathrm{MHz}, \mathrm{CDCl}_{3}\right) \delta 50.0\left(\mathrm{CH}_{2}\right), 53.0\left(\mathrm{CH}_{3}\right), 108.5\left(\mathrm{C}_{\mathrm{q}}\right), 124.9\left(\mathrm{CH}_{\text {aro }}\right), 125.5\left(\mathrm{C}_{\mathrm{q}}\right), 126.0\left(\mathrm{CH}_{\text {aro }}\right)$, $127.1\left(\mathrm{C}_{\mathrm{q}}\right), 127.7\left(\mathrm{C}_{\mathrm{q}}\right), 127.9\left(\mathrm{CH}_{\text {aro }}\right), 128.0\left(2 \mathrm{CH}_{\text {aro }}\right), 129.1\left(2 \mathrm{CH}_{\text {aro }}\right), 129.3\left(\mathrm{CH}_{\text {aro }}\right), 129.4$ $\left(2 \mathrm{CH}_{\text {aro }}\right), 130.1\left(\mathrm{CH}_{\text {aro }}\right), 130.4\left(\mathrm{CH}_{\text {aro }}\right), 132.2\left(\mathrm{CH}_{\mathrm{aro}}\right), 134.7\left(\mathrm{C}_{\mathrm{q}}\right), 134.9\left(\mathrm{C}_{\mathrm{q}}\right), 138.9\left(\mathrm{C}_{\mathrm{q}}\right), 143.9$ $\left(\mathrm{C}_{\mathrm{q}}\right), 149.7\left(\mathrm{C}_{\mathrm{q}}\right), 152.1\left(\mathrm{C}_{\mathrm{q}}\right), 160.7(\mathrm{C}=\mathrm{O}), 167.4(\mathrm{C}=\mathrm{O})$. Anal. Calcd for $\mathrm{C}_{27} \mathrm{H}_{18} \mathrm{~N}_{2} \mathrm{O}_{3}$ (418.13): $\mathrm{C}$ 77.50, H 4.34, N 6.69. Found: C 77.29, H 4.22, N 6.55.

a General protocol for the ester hydrolysis and ensuing decarboxylation 10g-l. A solution of aromathecins $10 \mathrm{~g}-\mathrm{l}(1 \mathrm{mmol})$ in $7 \mathrm{~mL}$ of $48 \%$ hydrobromic acid $(\mathrm{HBr})$ was heated under stirring at $135^{\circ} \mathrm{C}$ for $12 \mathrm{~h}$. After cooling at room temperature, the solution was alkalinized slowly and stepwise under stirring with saturated solution of sodium hydrogenocarbonate and extracted with dichloromethane. The organic layers combined were dried over anhydrous $\mathrm{MgSO}_{4}$, filtered and concentrated under reduce pressure to provide pure products $\mathbf{2 g}$ and $\mathbf{2 h}-\mathbf{l}$ in yields ranging from 51 up to $86 \%$.

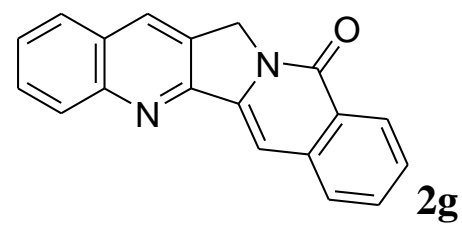

12H-5,11a-Diazadibenzo[b,h]fluoren-11-one (Rosettacin: $\mathbf{2 g}$ ). This product was isolated as a yellow solid in $61 \%$ yield $\left(171 \mathrm{mg}\right.$ ); $R_{f} 0.19$ (cyclohexane/AcOEt $1: 1$ ); mp $288^{\circ} \mathrm{C}$ (lit. ${ }^{[1]}$ 289$291^{\circ} \mathrm{C}$; lit. $\left.^{[2]} 286^{\circ} \mathrm{C}\right)$; IR (KBr) $v 1663,1628,1602,1504,1479,1398,1384,1239,1216,1166 \mathrm{~cm}^{-}$ ${ }^{1} ;{ }^{1} \mathrm{H}$ NMR $\left(200 \mathrm{MHz}, \mathrm{CDCl}_{3}\right) \delta 5.31\left(\mathrm{~s}, 2 \mathrm{H}, \mathrm{CH}_{2} \mathrm{~N}\right), 7.49-7.65\left(\mathrm{~m}, 3 \mathrm{H}, \mathrm{H}_{\text {aro }}\right), 7.68-7.86(\mathrm{~m}, 4 \mathrm{H}$, $\left.\mathrm{H}_{\text {aro }}\right), 8.17\left(\mathrm{~d}, 1 \mathrm{H}, \mathrm{H}_{\text {aro }}, J=7.8 \mathrm{~Hz}\right), 8.26\left(\mathrm{~s}, 1 \mathrm{H}, \mathrm{H}_{\mathrm{aro}}\right), 8.49\left(\mathrm{~d}, 1 \mathrm{H}, \mathrm{H}_{\text {aro }}, J=7.8 \mathrm{~Hz}\right) ;{ }^{13} \mathrm{C} \mathrm{NMR}(50$ $\left.\mathrm{MHz}, \mathrm{CDCl}_{3}\right) \delta 49.7\left(\mathrm{CH}_{2}\right), 101.6\left(\mathrm{CH}_{\text {aro }}\right), 126.3\left(\mathrm{C}_{\mathrm{q}}\right), 127.6\left(2 \mathrm{CH}_{\text {aro }}\right), 127.7\left(\mathrm{CH}_{\text {aro }}\right), 127.8$ $\left(\mathrm{CH}_{\text {aro }}\right), 128.2\left(\mathrm{C}_{\mathrm{q}}\right), 128.3\left(\mathrm{CH}_{\text {aro }}\right), 129.0\left(\mathrm{C}_{\mathrm{q}}\right), 129.6\left(\mathrm{CH}_{\text {aro }}\right), 130.5\left(\mathrm{CH}_{\text {aro }}\right), 131.1\left(\mathrm{CH}_{\text {aro }}\right), 132.7$ $\left(\mathrm{CH}_{\text {aro }}\right), 137.7\left(\mathrm{C}_{\mathrm{q}}\right), 140.1\left(\mathrm{C}_{\mathrm{q}}\right), 148.9\left(\mathrm{C}_{\mathrm{q}}\right), 153.7\left(\mathrm{C}_{\mathrm{q}}\right), 161.2(\mathrm{C}=\mathrm{O})$. Anal. Calcd for $\mathrm{C}_{19} \mathrm{H}_{12} \mathrm{~N}_{2} \mathrm{O}$ (284.09): C 80.27, H 4.25, N 9.85. Found: C 80.11, H 4.09, N 9.77.

\footnotetext{
${ }^{[1]}$ J. Warneke, E. Winterfeld, Chem. Ber. 1972, 105, 2120-2124.

${ }^{[2]}$ H. G. M. Walraven, U. K. Pandit, Tetrahedron 1980, 36, 321-327.
} 


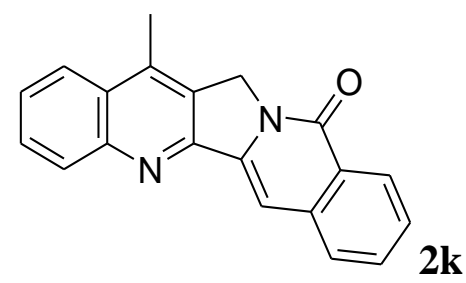

13-Methyl-12 $H-5,11 a-d i a z a d i b e n z o[b, h]$ fluoren-11-one $(\mathbf{2 k})$. This product was isolated as a yellow solid in $51 \%$ yield $\left(152 \mathrm{mg}\right.$ ); $R_{f} 0.30$ (cyclohexane/AcOEt $\left.1: 1\right) ; \mathrm{mp} 287^{\circ} \mathrm{C}$; IR $(\mathrm{KBr}) v$ $1662,1629,1602,1509,1478,1396,1341,1303,1291,1235,1216,1181 \mathrm{~cm}^{-1} ;{ }^{1} \mathrm{H}$ NMR (200 $\mathrm{MHz}, \mathrm{CDCl}_{3}$, the ${ }^{1} \mathrm{H} \mathrm{NMR}$ spectra contains traces of water) $\delta 2.65\left(\mathrm{~s}, 3 \mathrm{H}, \mathrm{CH}_{3}\right), 5.16(\mathrm{~s}, 2 \mathrm{H}$, $\mathrm{CH}_{2} \mathrm{~N}$ ), 7.45-7.59 (m, 3H, $\mathrm{H}_{\text {aro }}$ ), 7.60-7.74 (m, 3H, $\left.\mathrm{H}_{\text {aro }}\right), 7.93\left(\mathrm{~d}, 1 \mathrm{H}, \mathrm{H}_{\text {aro }}, J=8.6 \mathrm{~Hz}\right), 8.12(\mathrm{~d}$, $\left.1 \mathrm{H}, \mathrm{H}_{\text {aro }}, J=8.6 \mathrm{~Hz}\right), 8.44\left(\mathrm{~d}, 1 \mathrm{H}, \mathrm{H}_{\text {aro }}, J=7.8 \mathrm{~Hz}\right) ;{ }^{13} \mathrm{C} \mathrm{NMR}\left(50 \mathrm{MHz}, \mathrm{CDCl}_{3}\right) \delta 15.4\left(\mathrm{CH}_{3}\right)$, $49.3\left(\mathrm{CH}_{2}\right), 101.3\left(\mathrm{CH}_{\text {aro }}\right), 123.7\left(\mathrm{CH}_{\text {aro }}\right), 126.1\left(\mathrm{C}_{\mathrm{q}}\right), 127.2\left(\mathrm{CH}_{\text {aro }}\right), 127.4\left(\mathrm{CH}_{\text {aro }}\right), 127.6\left(2 \mathrm{CH}_{\text {aro }}\right)$, $128.0\left(\mathrm{C}_{\mathrm{q}}\right), 130.0\left(\mathrm{CH}_{\text {aro }}\right), 130.1\left(\mathrm{CH}_{\text {aro }}\right), 132.5\left(\mathrm{CH}_{\text {aro }}\right), 137.7\left(2 \mathrm{C}_{\mathrm{q}}\right), 139.5\left(\mathrm{C}_{\mathrm{q}}\right), 140.6\left(\mathrm{C}_{\mathrm{q}}\right), 148.8$ $\left(\mathrm{C}_{\mathrm{q}}\right), 152.8\left(\mathrm{C}_{\mathrm{q}}\right), 161.1(\mathrm{C}=\mathrm{O})$. Anal. Calcd for $\mathrm{C}_{20} \mathrm{H}_{14} \mathrm{~N}_{2} \mathrm{O}$ (298.11): C 80.52, H 4.73, N 9.39. Found: C 80.39, H 4.57, N 9.11.

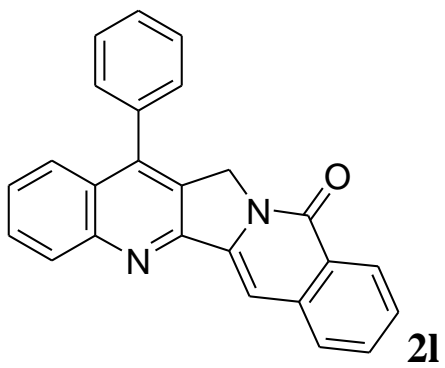

13-Phenyl-12 $H-5,11 a-d i a z a d i b e n z o[b, h]$ fluoren-11-one (2I). This product was isolated as a yellow solid in $86 \%$ yield $\left(305 \mathrm{mg}\right.$ ); $R_{f} 0.50$ (cyclohexane/AcOEt $\left.1: 1\right) ; \mathrm{mp}>300^{\circ} \mathrm{C}$; IR $(\mathrm{KBr}) v$ 1661, 1628, 1604, 1478, 1396, 1344, 1217, $1166 \mathrm{~cm}^{-1} ;{ }^{1} \mathrm{H}$ NMR $\left(200 \mathrm{MHz}, \mathrm{CDCl}_{3}\right.$, the ${ }^{1} \mathrm{H} \mathrm{NMR}$ spectra contains traces of water and acetone) $\delta 5.16\left(\mathrm{~s}, 2 \mathrm{H}, \mathrm{CH}_{2} \mathrm{~N}\right), 7.44-7.62\left(\mathrm{~m}, 7 \mathrm{H}, \mathrm{H}_{\text {aro }}\right), 7.66$ (s, $\left.1 \mathrm{H}, \mathrm{H}_{\text {aro }}\right), 7.70-7.80\left(\mathrm{~m}, 4 \mathrm{H}, \mathrm{H}_{\text {aro }}\right), 8.24\left(\mathrm{~d}, 1 \mathrm{H}, \mathrm{H}_{\text {aro }}, J=7.8 \mathrm{~Hz}\right), 8.49$ (d, $\left.1 \mathrm{H}, \mathrm{H}_{\text {aro }}, J=7.8 \mathrm{~Hz}\right)$; ${ }^{13} \mathrm{C}$ NMR $\left(75 \mathrm{MHz}, \mathrm{CDCl}_{3}\right) \delta 49.8\left(\mathrm{CH}_{2}\right), 101.4\left(\mathrm{CH}_{\mathrm{aro}}\right), 126.2\left(\mathrm{CH}_{\mathrm{aro}}\right), 126.3\left(\mathrm{C}_{\mathrm{q}}\right), 127.2\left(\mathrm{C}_{\mathrm{q}}\right)$, $127.4\left(\mathrm{CH}_{\text {aro }}\right), 127.5\left(\mathrm{CH}_{\text {aro }}\right), 127.7\left(\mathrm{CH}_{\text {aro }}\right.$ and $\left.\mathrm{C}_{\mathrm{q}}\right), 127.8\left(\mathrm{CH}_{\text {aro }}\right), 129.1\left(2 \mathrm{CH}_{\text {aro }}\right), 129.3\left(\mathrm{CH}_{\text {aro }}\right)$, $129.4\left(2 \mathrm{CH}_{\text {aro }}\right), 129.9\left(\mathrm{CH}_{\text {aro }}\right), 130.2\left(\mathrm{CH}_{\text {aro }}\right), 132.6\left(\mathrm{CH}_{\text {aro }}\right), 135.1\left(\mathrm{C}_{\mathrm{q}}\right), 137.8\left(\mathrm{C}_{\mathrm{q}}\right), 140.7\left(\mathrm{C}_{\mathrm{q}}\right)$, 144.1 $\left(\mathrm{C}_{\mathrm{q}}\right), 149.7\left(\mathrm{C}_{\mathrm{q}}\right), 153.3\left(\mathrm{C}_{\mathrm{q}}\right), 161.2(\mathrm{C}=\mathrm{O})$. Anal. Calcd for $\mathrm{C}_{25} \mathrm{H}_{16} \mathrm{~N}_{2} \mathrm{O}$ (360.13): $\mathrm{C}$ 83.31, $\mathrm{H}$ 4.47, N 7.77. Found: C 83.09, H 4.31, N 7.54.

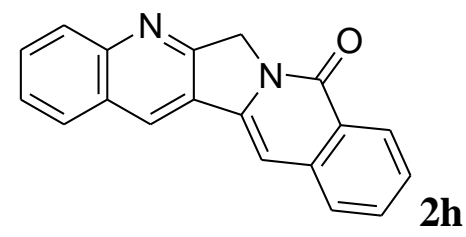

12H-11,12a-Diazadibenzo[b,h]fluoren-13-one (Isorosettacin: $2 \mathrm{~h}$ ). This product was isolated as a brown solid in $67 \%$ yield $\left(190 \mathrm{mg}\right.$ ); $R_{f} 0.24$ (cyclohexane/AcOEt $\left.1: 1\right) ; \mathrm{mp} 292^{\circ} \mathrm{C}$; IR (KBr) $v$ 1662, 1627, 1603, 1503, 1472, 1344, 1296, 1239, 1216, $1167 \mathrm{~cm}^{-1} ;{ }^{1} \mathrm{H}$ NMR $\left(200 \mathrm{MHz}, \mathrm{CDCl}_{3}\right.$, the ${ }^{1} \mathrm{H}$ NMR spectra contains traces of water and acetone) $\delta 5.34\left(\mathrm{~s}, 2 \mathrm{H}, \mathrm{CH}_{2} \mathrm{~N}\right), 7.18\left(\mathrm{~s}, 1 \mathrm{H}, \mathrm{H}_{\mathrm{aro}}\right)$, 7.46-7.56 (m, $\left.1 \mathrm{H}, \mathrm{H}_{\text {aro }}\right), 7.60-7.68\left(\mathrm{~m}, 3 \mathrm{H}, \mathrm{H}_{\text {aro }}\right), 7.77\left(\mathrm{ddd}, 1 \mathrm{H}, \mathrm{H}_{\text {aro }}, J=8.6,7.0\right.$ and $\left.1.6 \mathrm{~Hz}\right), 7.93$ $\left(\mathrm{d}, 1 \mathrm{H}, \mathrm{H}_{\text {aro }}, J=7.8 \mathrm{~Hz}\right), 8.12\left(\mathrm{~d}, 1 \mathrm{H}, \mathrm{H}_{\text {aro }}, J=8.6 \mathrm{~Hz}\right), 8.48-8.51\left(\mathrm{~m}, 2 \mathrm{H}, \mathrm{H}_{\text {aro }}\right) ;{ }^{13} \mathrm{C}$ NMR $(75$ $\left.\mathrm{MHz}, \mathrm{CDCl}_{3}\right) \delta 52.6\left(\mathrm{CH}_{2}\right), 100.5\left(\mathrm{CH}_{\text {aro }}\right), 125.7\left(\mathrm{C}_{\mathrm{q}}\right), 126.3\left(\mathrm{C}_{\mathrm{q}}\right), 126.8\left(\mathrm{CH}_{\text {aro }}\right), 127.2\left(\mathrm{CH}_{\text {aro }}\right)$, $127.5\left(\mathrm{CH}_{\text {aro }}\right), 127.6\left(\mathrm{C}_{\mathrm{q}}\right), 127.9\left(\mathrm{CH}_{\text {aro }}\right), 128.5\left(\mathrm{CH}_{\text {aro }}\right), 128.8\left(\mathrm{CH}_{\text {aro }}\right), 129.1\left(\mathrm{C}_{\mathrm{q}}\right), 129.4\left(\mathrm{CH}_{\text {aro }}\right)$, 
$131.0\left(\mathrm{CH}_{\text {aro }}\right), 132.7\left(\mathrm{CH}_{\text {aro }}\right), 137.6\left(\mathrm{C}_{\mathrm{q}}\right), 139.8\left(\mathrm{C}_{\mathrm{q}}\right), 158.9\left(\mathrm{C}_{\mathrm{q}}\right), 161.1(\mathrm{C}=\mathrm{O})$. Anal. Calcd for $\mathrm{C}_{19} \mathrm{H}_{12} \mathrm{~N}_{2} \mathrm{O}$ (284.09): C 80.27, H 4.25, N 9.85. Found: C 80.09, H 4.11, N 9.69.

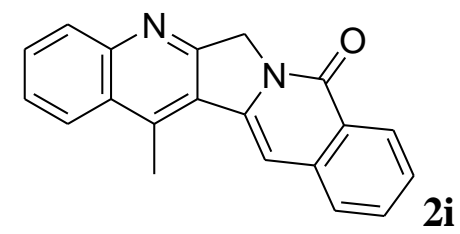

6-Methyl-12H-11,12a-diazadibenzo $[\boldsymbol{b}, \boldsymbol{h}]$ fluoren-13-one (2i). This product was isolated as a brown solid in $65 \%$ yield $\left(193 \mathrm{mg}\right.$ ); $R_{f} 0.38$ (cyclohexane/AcOEt $\left.3: 7\right)$; mp $290^{\circ} \mathrm{C}$; IR $(\mathrm{KBr}) v$ 1776, 1736, 1652, 1624, 1601, 1557, 1336, 1280, 1217, 1173, $1138 \mathrm{~cm}^{-1}$; ${ }^{1} \mathrm{H}$ NMR $(300 \mathrm{MHz}$, $\mathrm{CDCl}_{3}$, the ${ }^{1} \mathrm{H} \mathrm{NMR}$ spectra contains traces of water and acetone) $\delta 3.02\left(\mathrm{~s}, 3 \mathrm{H}, \mathrm{CH}_{3}\right), 5.27(\mathrm{~s}, 2 \mathrm{H}$, $\left.\mathrm{CH}_{2} \mathrm{~N}\right), 7.21\left(\mathrm{~s}, 1 \mathrm{H}, \mathrm{H}_{\text {aro }}\right), 7.44-7.49\left(\mathrm{~m}, 1 \mathrm{H}, \mathrm{H}_{\text {aro }}\right), 7.58-7.67\left(\mathrm{~m}, 3 \mathrm{H}, \mathrm{H}_{\text {aro }}\right), 7.71-7.76(\mathrm{~m}, 1 \mathrm{H}$, $\left.\mathrm{H}_{\text {aro }}\right), 8.09\left(\mathrm{~d}, 1 \mathrm{H}, \mathrm{H}_{\text {aro }}, J=8.2 \mathrm{~Hz}\right), 8.19\left(\mathrm{~d}, 1 \mathrm{H}, \mathrm{H}_{\text {aro }}, J=8.5 \mathrm{~Hz}\right), 8.45\left(\mathrm{~d}, 1 \mathrm{H}, \mathrm{H}_{\text {aro }}, J=8.0 \mathrm{~Hz}\right)$; ${ }^{13} \mathrm{C}$ NMR $\left(75 \mathrm{MHz}, \mathrm{CDCl}_{3}\right) \delta 15.1\left(\mathrm{CH}_{3}\right), 52.3\left(\mathrm{CH}_{2}\right), 104.3\left(\mathrm{CH}_{\text {aro }}\right), 124.3\left(\mathrm{CH}_{\text {aro }}\right), 125.0\left(\mathrm{C}_{\mathrm{q}}\right)$, $127.1\left(2 \mathrm{CH}_{\text {aro }}\right), 127.3\left(\mathrm{CH}_{\text {aro }}\right), 127.5\left(\mathrm{CH}_{\text {aro }}\right.$ and $\left.\mathrm{C}_{\mathrm{q}}\right), 127.6\left(\mathrm{C}_{\mathrm{q}}\right), 129.8\left(\mathrm{CH}_{\text {aro }}\right), 130.6\left(\mathrm{CH}_{\text {aro }}\right)$, $132.6\left(\mathrm{CH}_{\text {aro }}\right), 137.7\left(\mathrm{C}_{\mathrm{q}}\right), 140.9\left(\mathrm{C}_{\mathrm{q}}\right), 141.0\left(\mathrm{C}_{\mathrm{q}}\right), 148.1\left(\mathrm{C}_{\mathrm{q}}\right), 158.4\left(\mathrm{C}_{\mathrm{q}}\right), 161.0(\mathrm{C}=\mathrm{O})$. Anal. Calcd for $\mathrm{C}_{20} \mathrm{H}_{14} \mathrm{~N}_{2} \mathrm{O}$ (298.11): C 80.52, H 4.73, N 9.39. Found: $\mathrm{C} 80.33, \mathrm{H} 4.64, \mathrm{~N} 9.09$.<smiles>O=c1c2ccccc2cc2n1Cc1nc3ccccc3c(-c3ccccc3)c1-2</smiles>

6-Phenyl-12H-11,12a-diazadibenzo $[\boldsymbol{b}, \boldsymbol{h}]$ fluoren-13-one $(\mathbf{2 j})$. This product was isolated as a yellow solid in $64 \%$ yield $\left(230 \mathrm{mg}\right.$ ); $R_{f} 0.30$ (cyclohexane/AcOEt $\left.1: 1\right) ; \mathrm{mp} 292^{\circ} \mathrm{C}$; IR $(\mathrm{KBr}) v$ $1778,1650,1617,1603,1578,1479,1384,1342,1280,1216,1175 \mathrm{~cm}^{-1} ;{ }^{1} \mathrm{H}$ NMR $(300 \mathrm{MHz}$, $\mathrm{CDCl}_{3}$, the ${ }^{1} \mathrm{H} \mathrm{NMR}$ spectra contains traces of water and $\left.\mathrm{Et}_{2} \mathrm{O}\right) \delta 5.35\left(\mathrm{~s}, 2 \mathrm{H}, \mathrm{CH}_{2} \mathrm{~N}\right), 5.90(\mathrm{~s}, 1 \mathrm{H}$, $\left.\mathrm{H}_{\text {aro }}\right)$, 7.21-7.24 (m, $\left.1 \mathrm{H}, \mathrm{H}_{\text {aro }}\right), 7.40-7.56\left(\mathrm{~m}, 5 \mathrm{H}, \mathrm{H}_{\text {aro }}\right), 7.61\left(\mathrm{~d}, 1 \mathrm{H}, \mathrm{H}_{\text {aro }}, J=8.1 \mathrm{~Hz}\right), 7.67-7.78$ $\left(\mathrm{m}, 4 \mathrm{H}, \mathrm{H}_{\mathrm{aro}}\right), 8.16\left(\mathrm{~d}, 1 \mathrm{H}, \mathrm{H}_{\text {aro }}, J=8.4 \mathrm{~Hz}\right), 8.42\left(\mathrm{~d}, 1 \mathrm{H}, \mathrm{H}_{\mathrm{aro}}, J=7.9 \mathrm{~Hz}\right) ;{ }^{13} \mathrm{C} \mathrm{NMR}(75 \mathrm{MHz}$, $\left.\mathrm{CDCl}_{3}\right) \delta 52.3\left(\mathrm{CH}_{2}\right), 103.7\left(\mathrm{CH}_{\text {aro }}\right), 123.7\left(\mathrm{C}_{\mathrm{q}}\right), 125.2\left(\mathrm{C}_{\mathrm{q}}\right), 126.8\left(\mathrm{CH}_{\text {aro }}\right), 127.1\left(\mathrm{CH}_{\text {aro }}\right), 127.2$ $\left(\mathrm{CH}_{\text {aro }}\right), 127.3\left(\mathrm{CH}_{\text {aro }}\right), 127.5\left(\mathrm{CH}_{\text {aro }}\right), 129.0\left(2 \mathrm{CH}_{\text {aro }}\right.$ and $\left.\mathrm{C}_{\mathrm{q}}\right), 129.3\left(\mathrm{CH}_{\text {aro }}\right), 129.5\left(\mathrm{CH}_{\text {aro }}\right), 129.6$ $\left(2 \mathrm{CH}_{\text {aro }}\right), 130.8\left(\mathrm{CH}_{\text {aro }}\right), 132.4\left(\mathrm{CH}_{\text {aro }}\right), 134.7\left(\mathrm{C}_{\mathrm{q}}\right), 137.4\left(\mathrm{C}_{\mathrm{q}}\right), 139.8\left(\mathrm{C}_{\mathrm{q}}\right), 144.5\left(\mathrm{C}_{\mathrm{q}}\right), 148.5\left(\mathrm{C}_{\mathrm{q}}\right)$, $158.7\left(\mathrm{C}_{\mathrm{q}}\right), 161.0(\mathrm{C}=\mathrm{O})$. Anal. Calcd for $\mathrm{C}_{25} \mathrm{H}_{16} \mathrm{~N}_{2} \mathrm{O}$ (360.13): C 83.31, H 4.47, N 7.77. Found: $\mathrm{C}$ 83.13, H 4.29, N 7.61. 


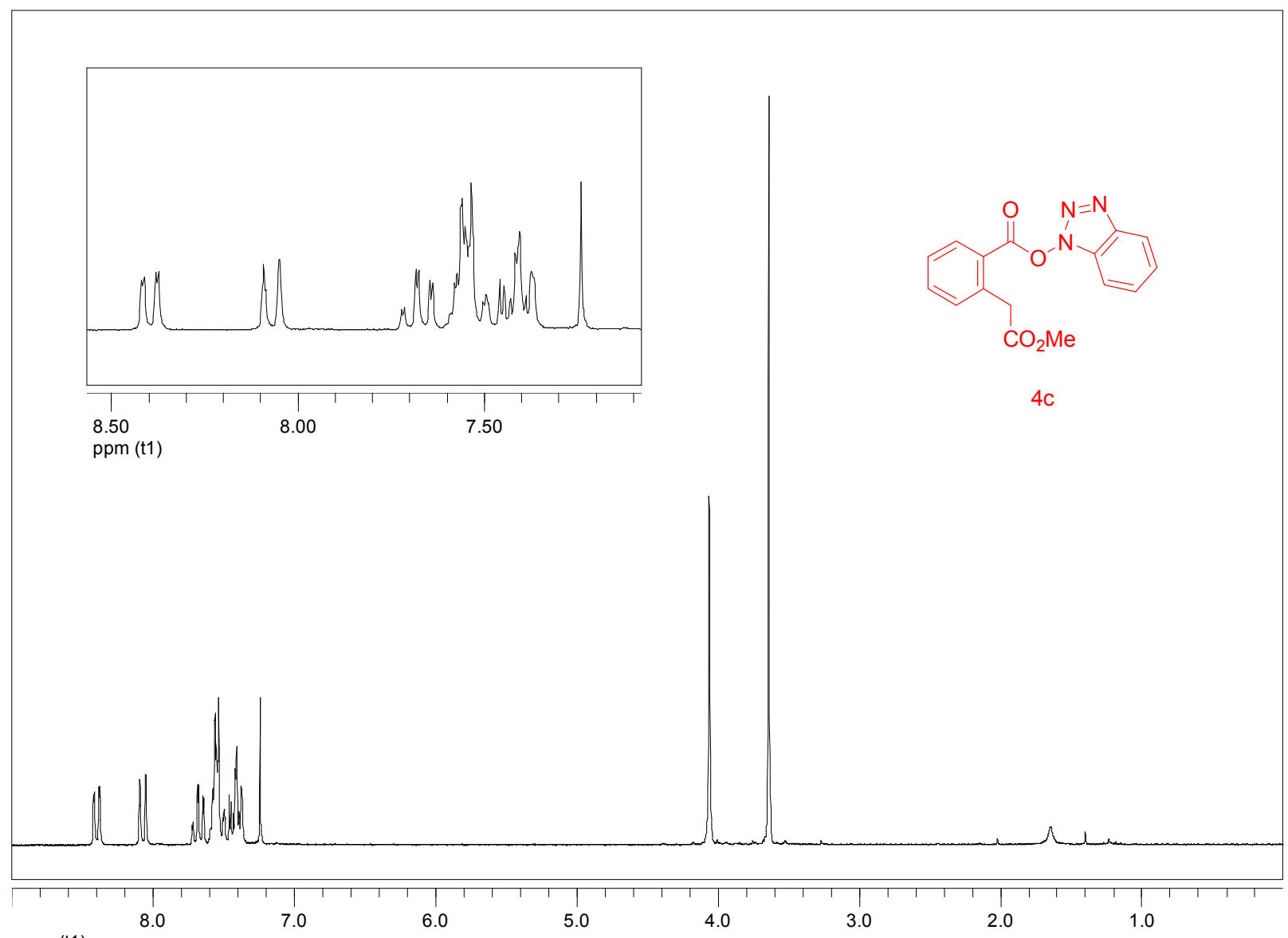

ppm (t1)

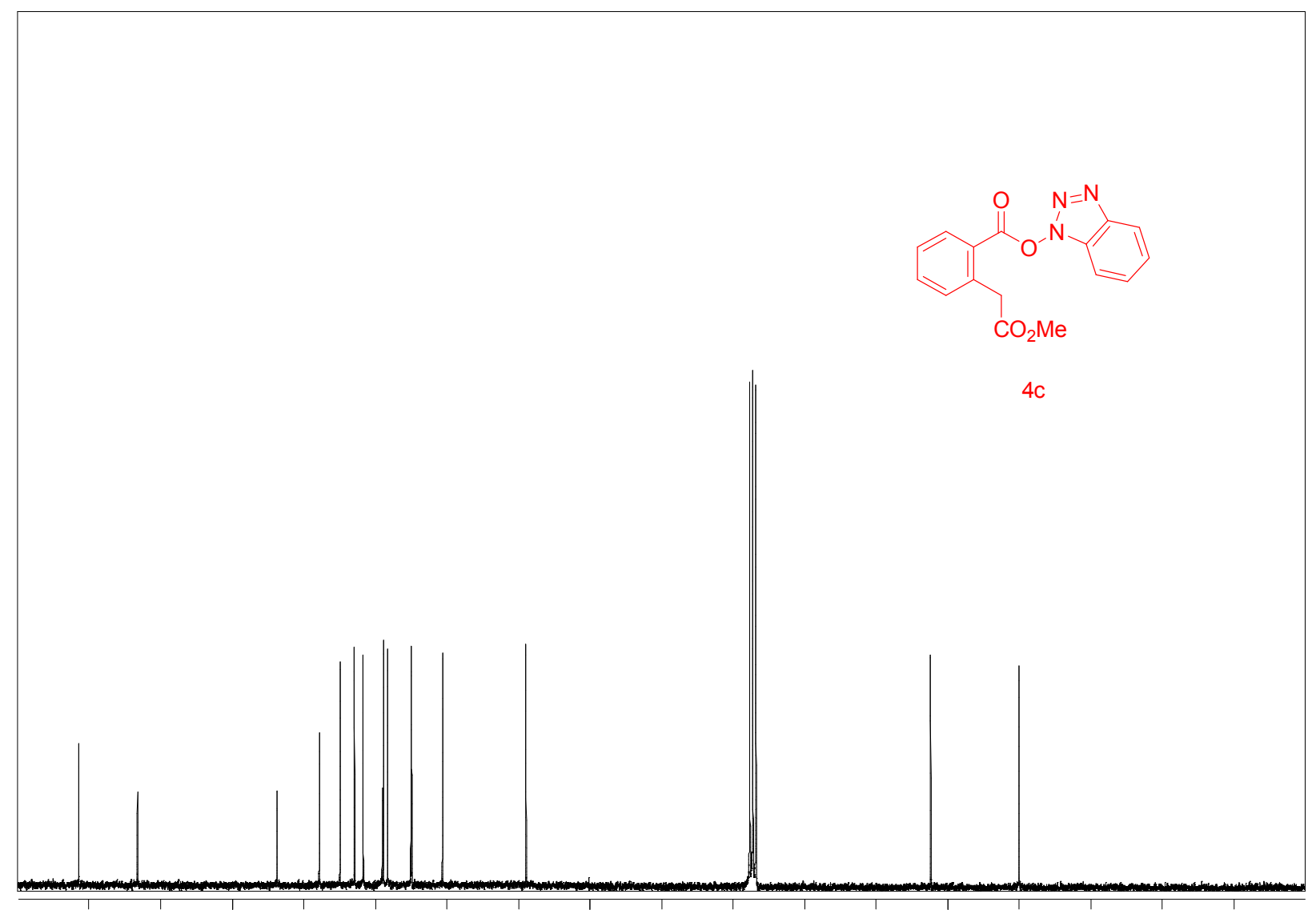

\begin{tabular}{l|l}
150 \\
\hline 1
\end{tabular}

ppm (t1)

100

50 

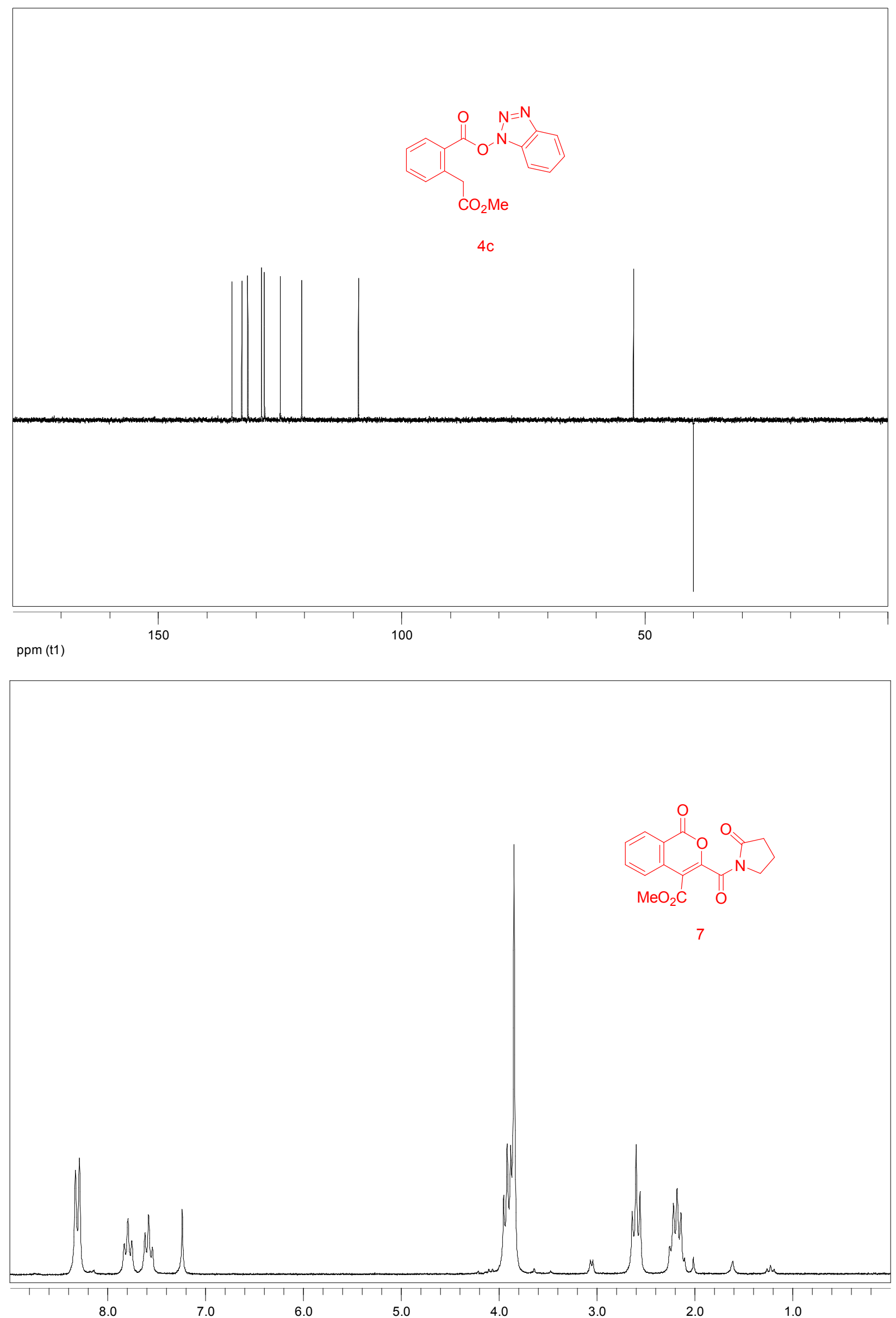

ppm (t1) 


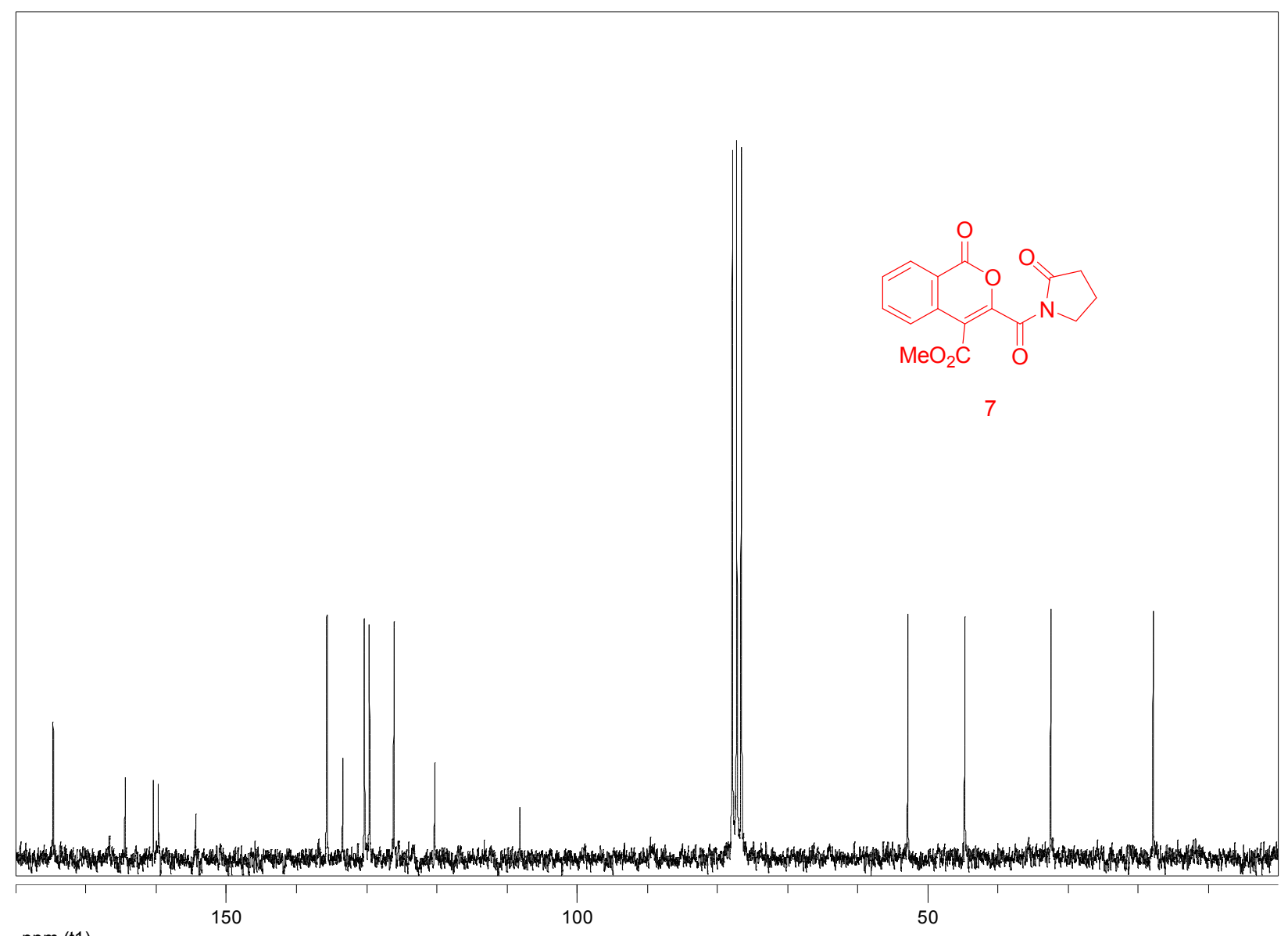

ppm (t1)

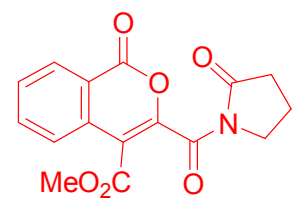

7

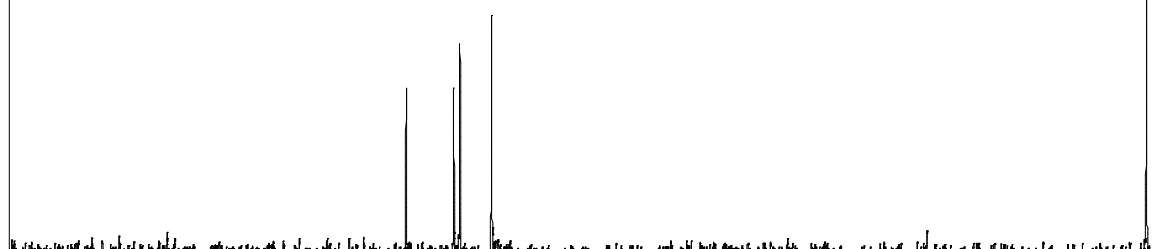

Wy

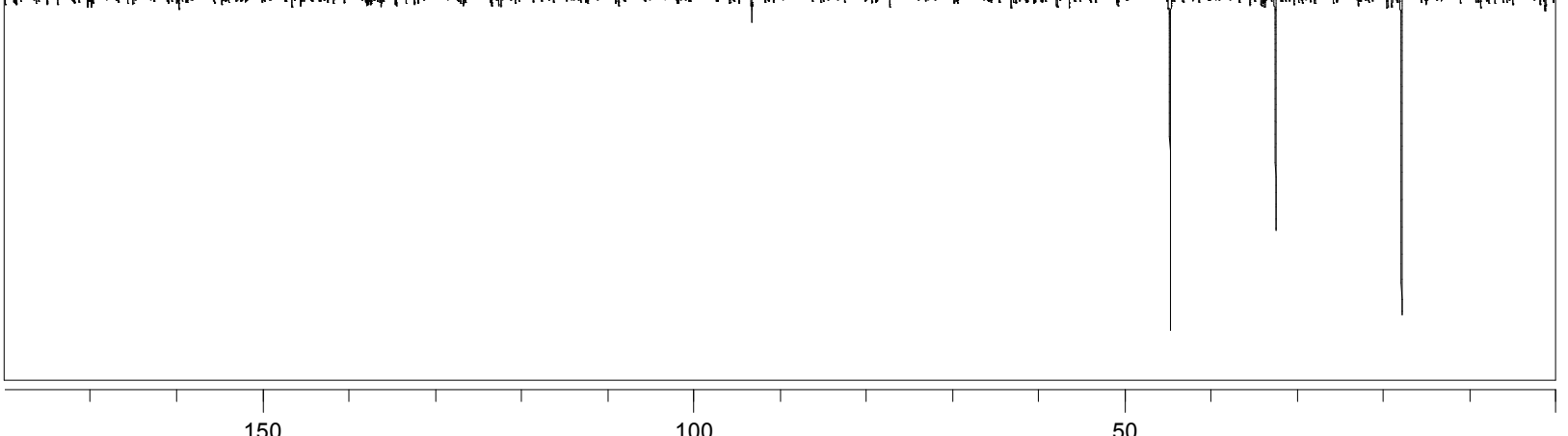

ppm (t1) 


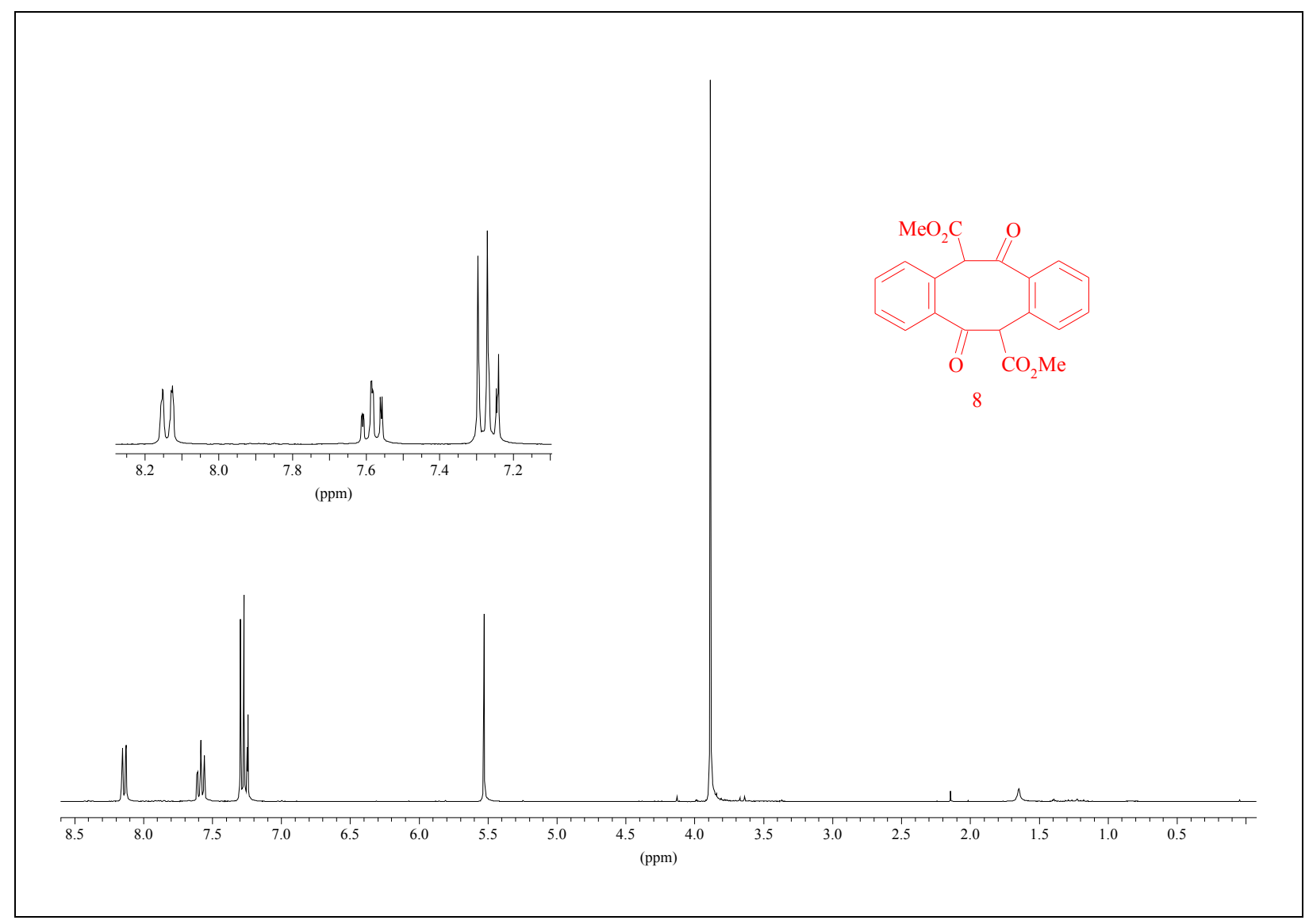

$\mathrm{C} 13 \mathrm{CDCl} 3$

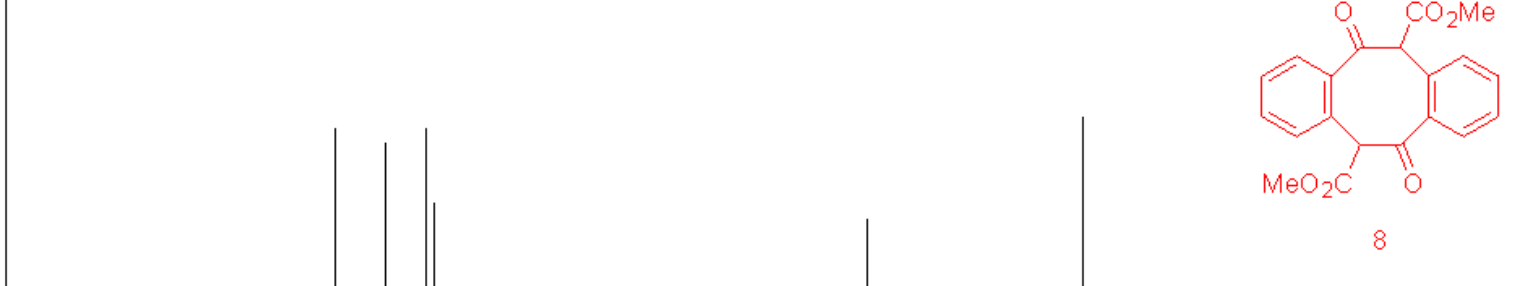


Dept $135 \mathrm{CDCl} 3$

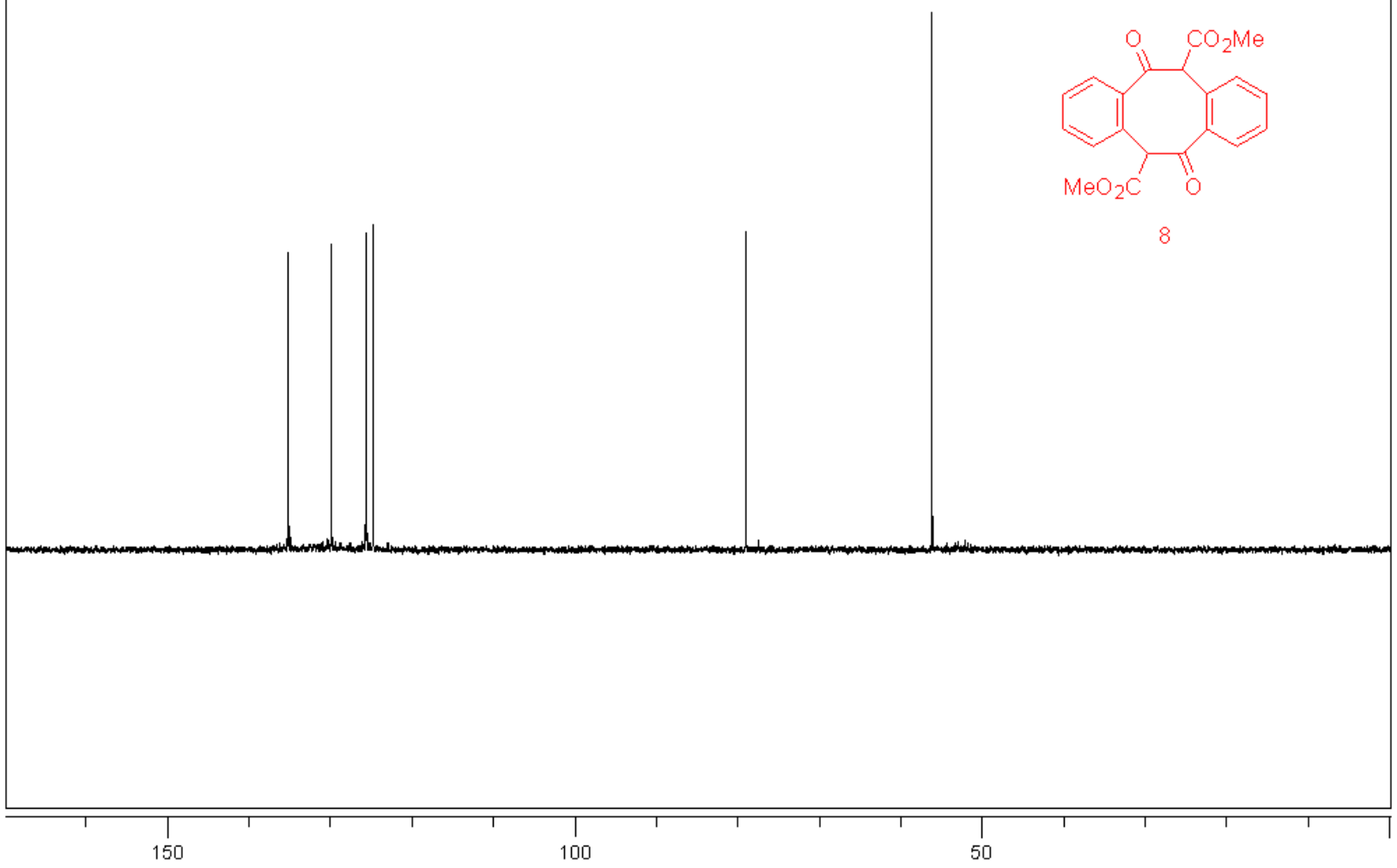

ppm (f1)

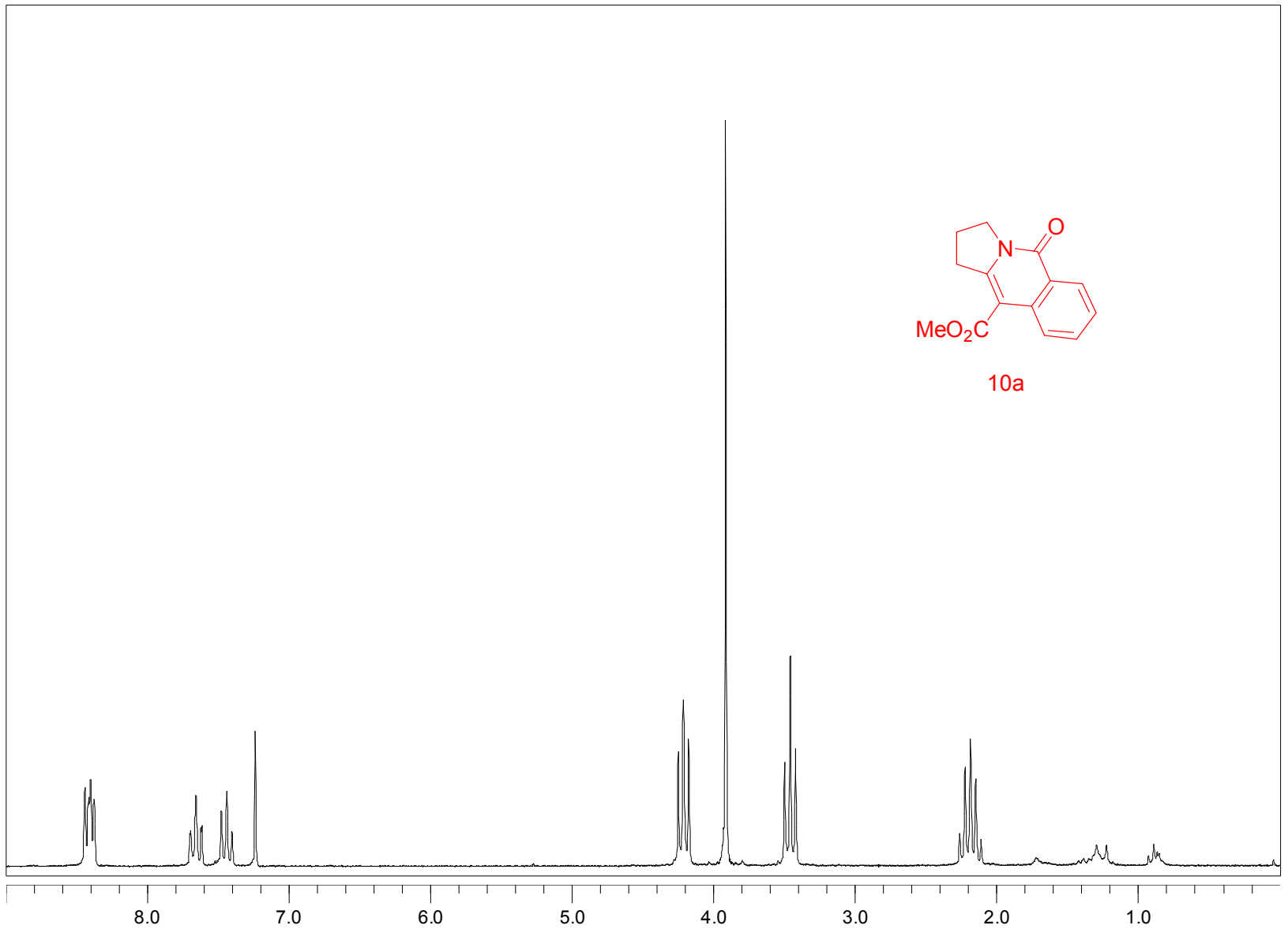

ppm (t1) 

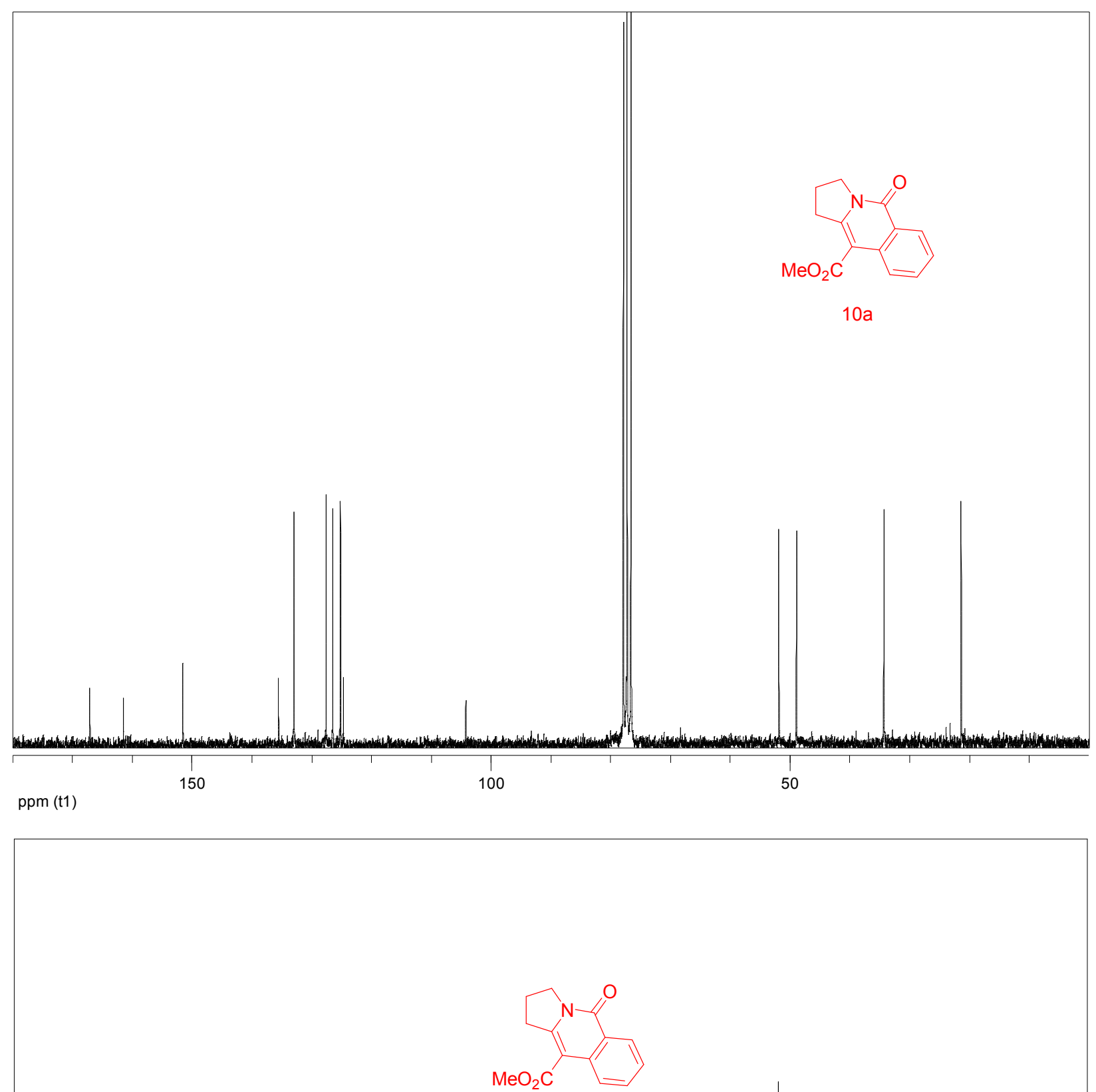

$10 a$
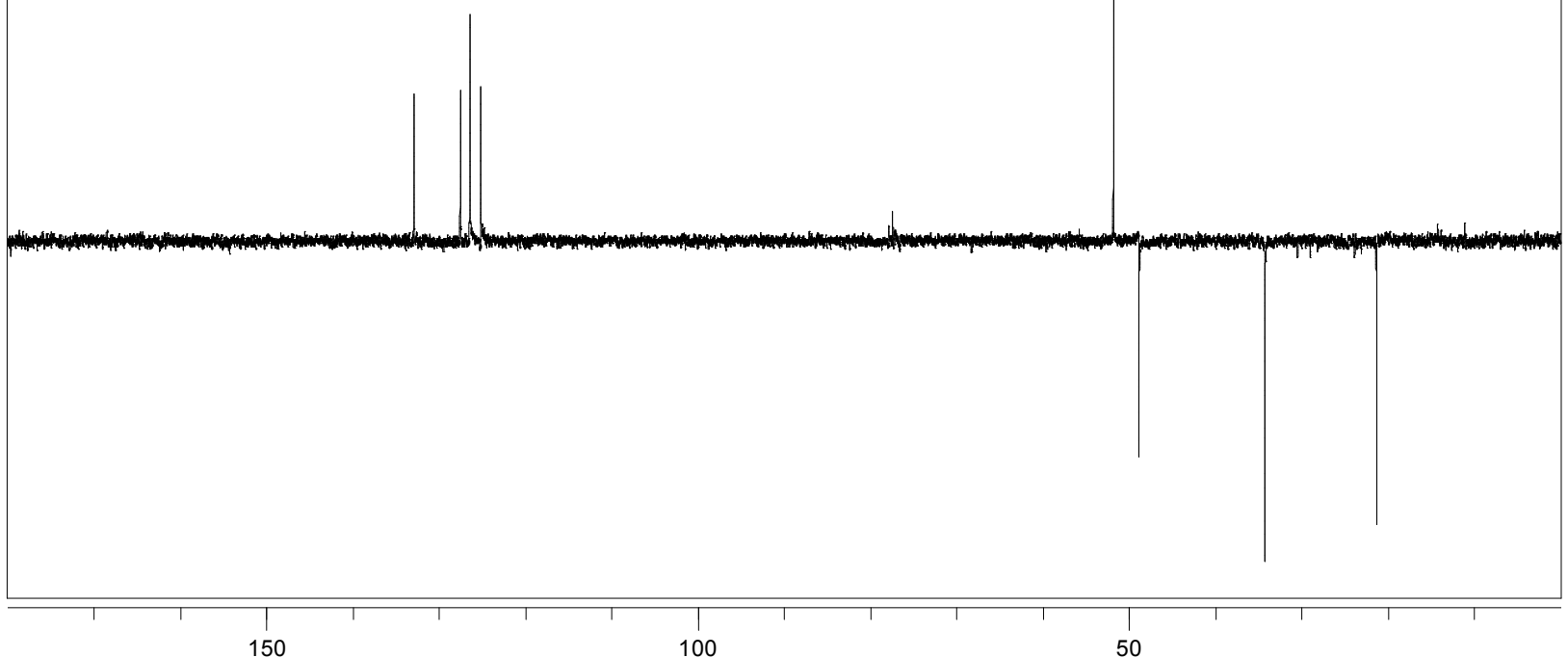

ppm (t1) 

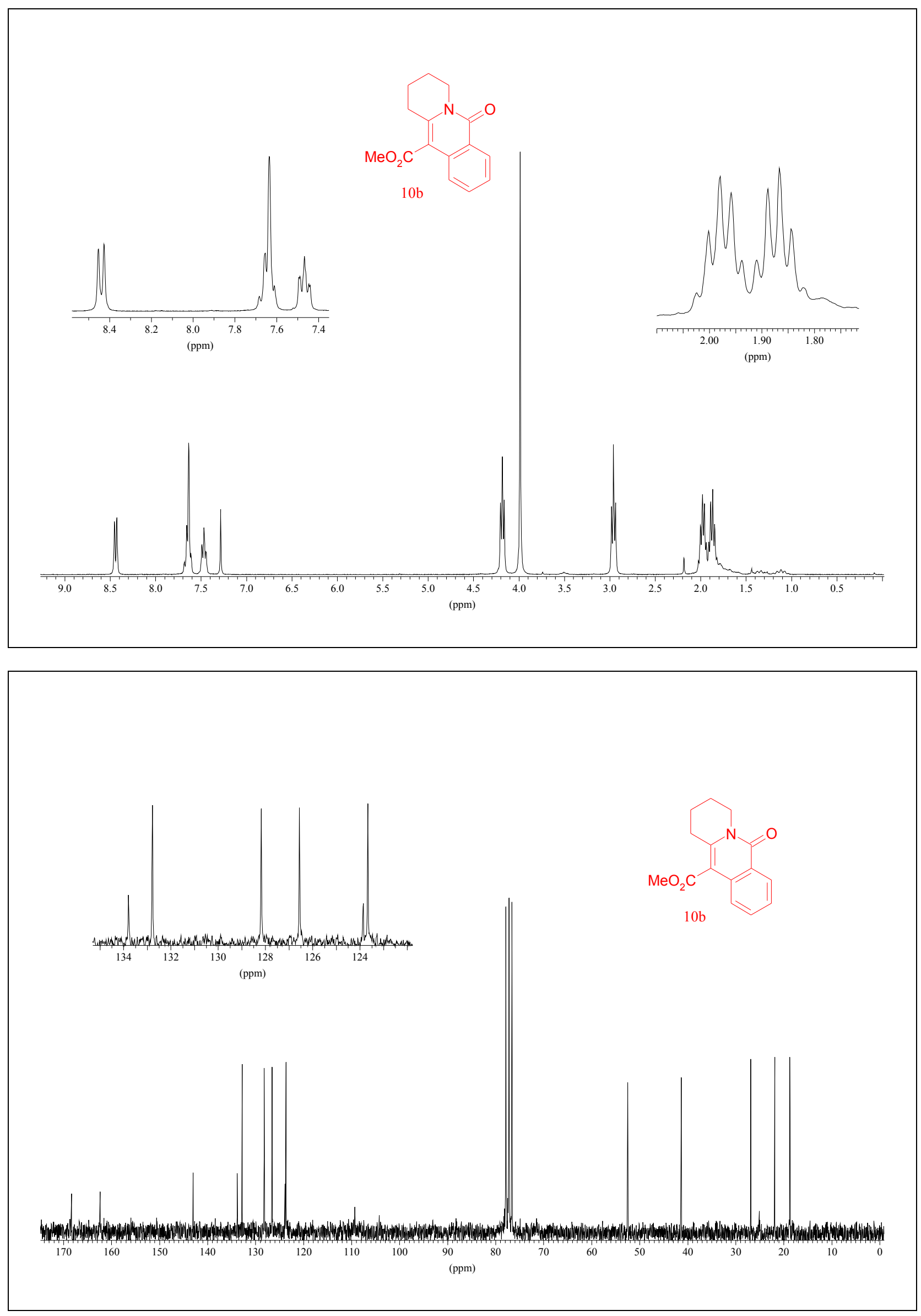

SI-19- 

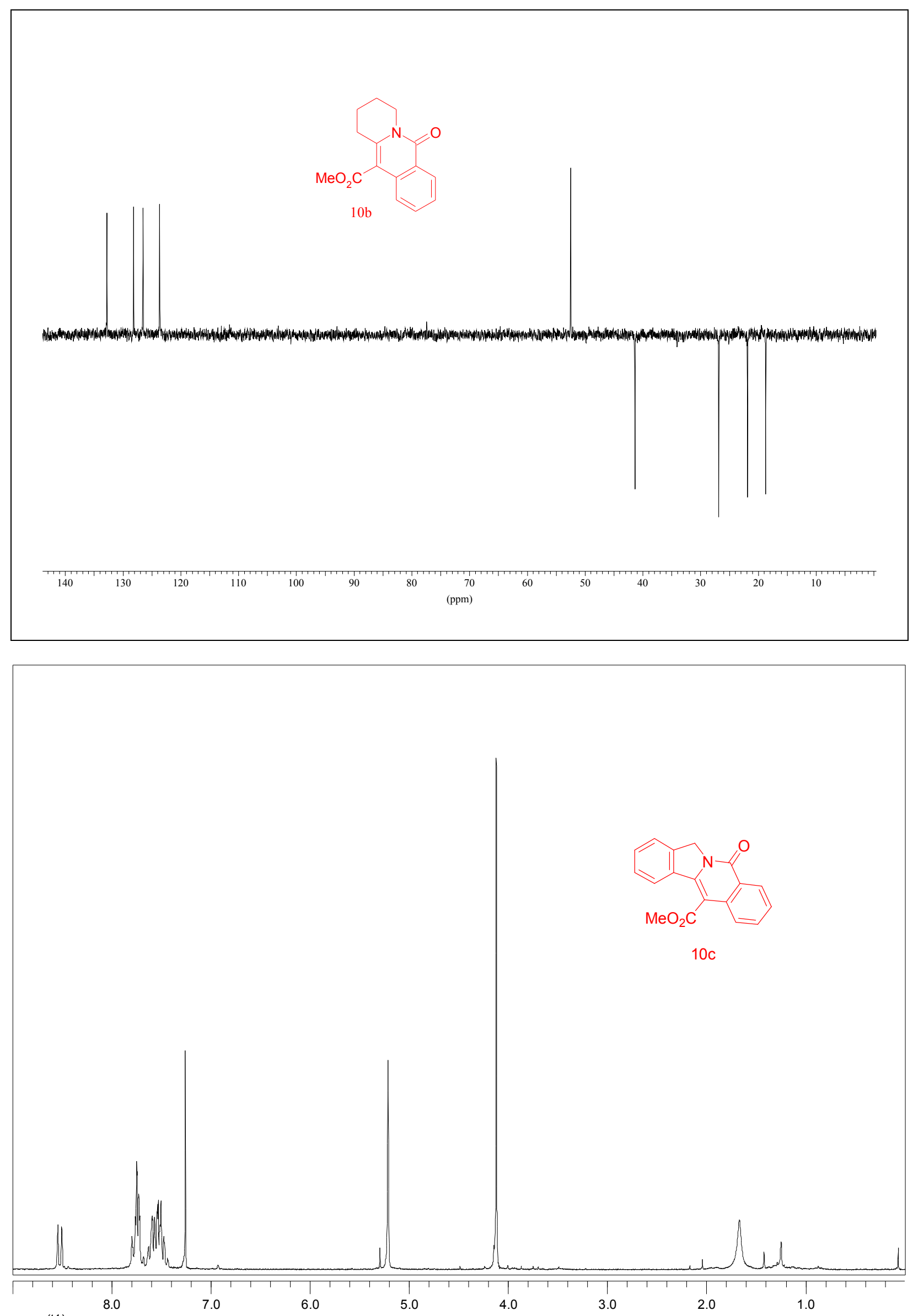

ppm (t1) 


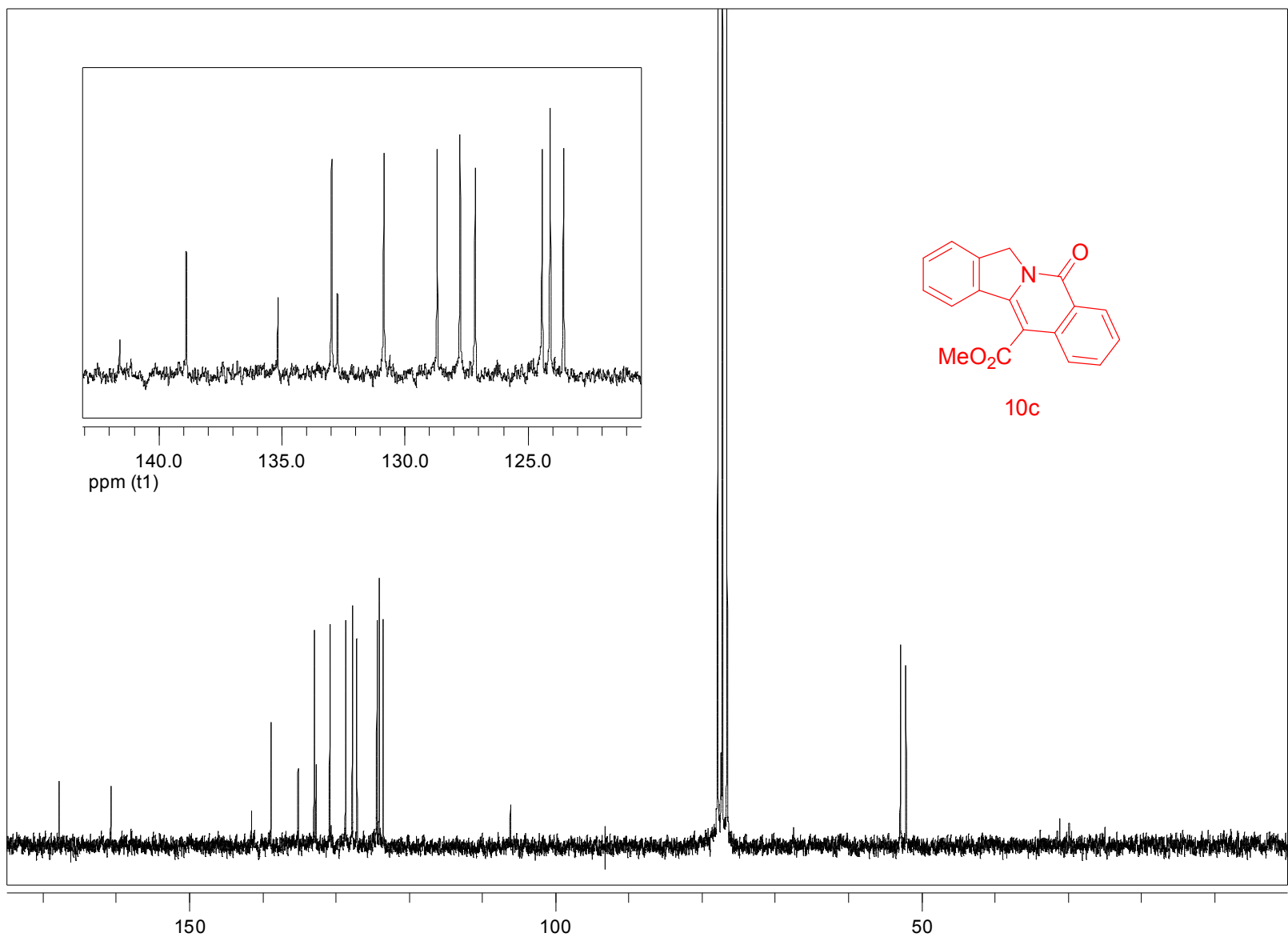

ppm (t1)
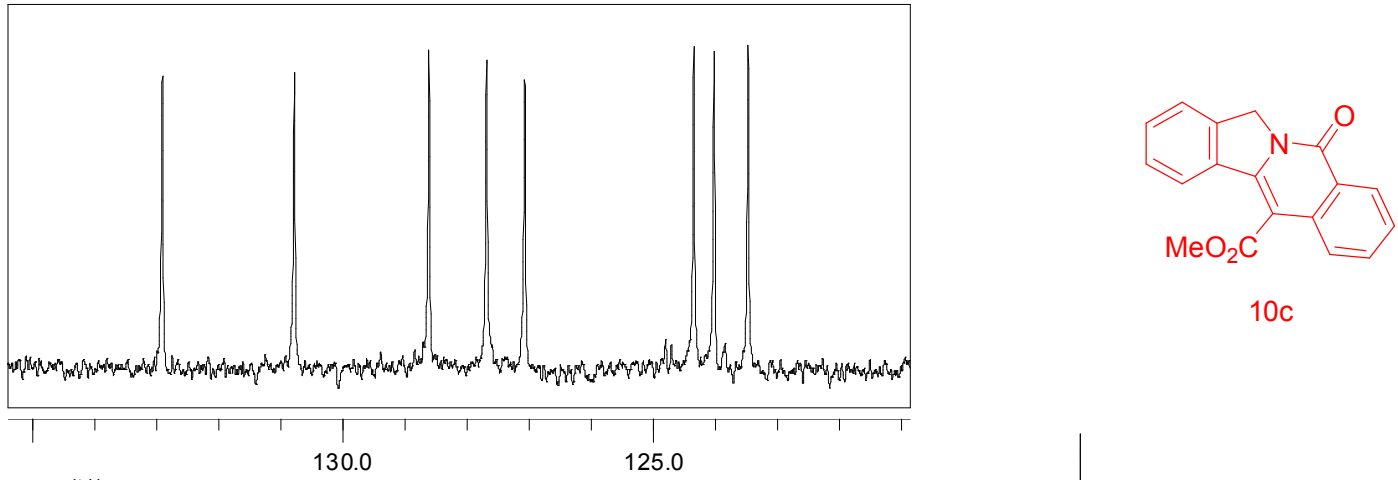

ppm (t1)
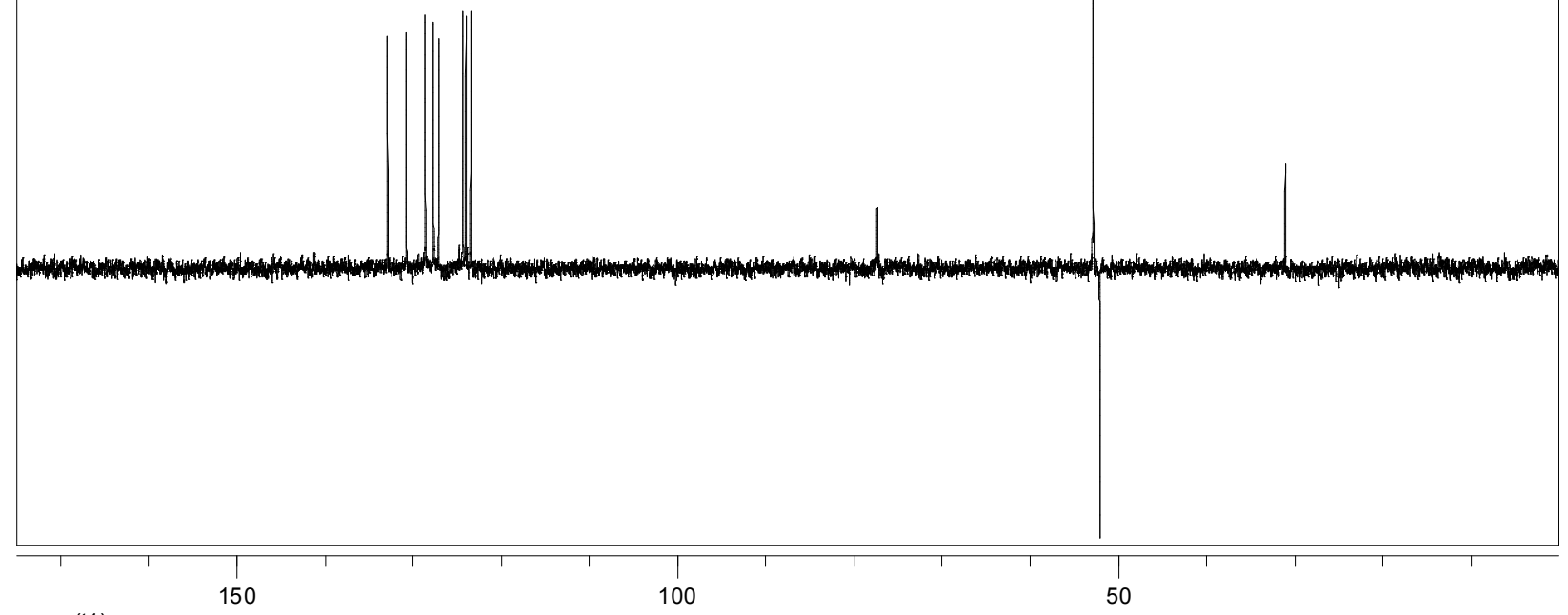

ppm (t1) 

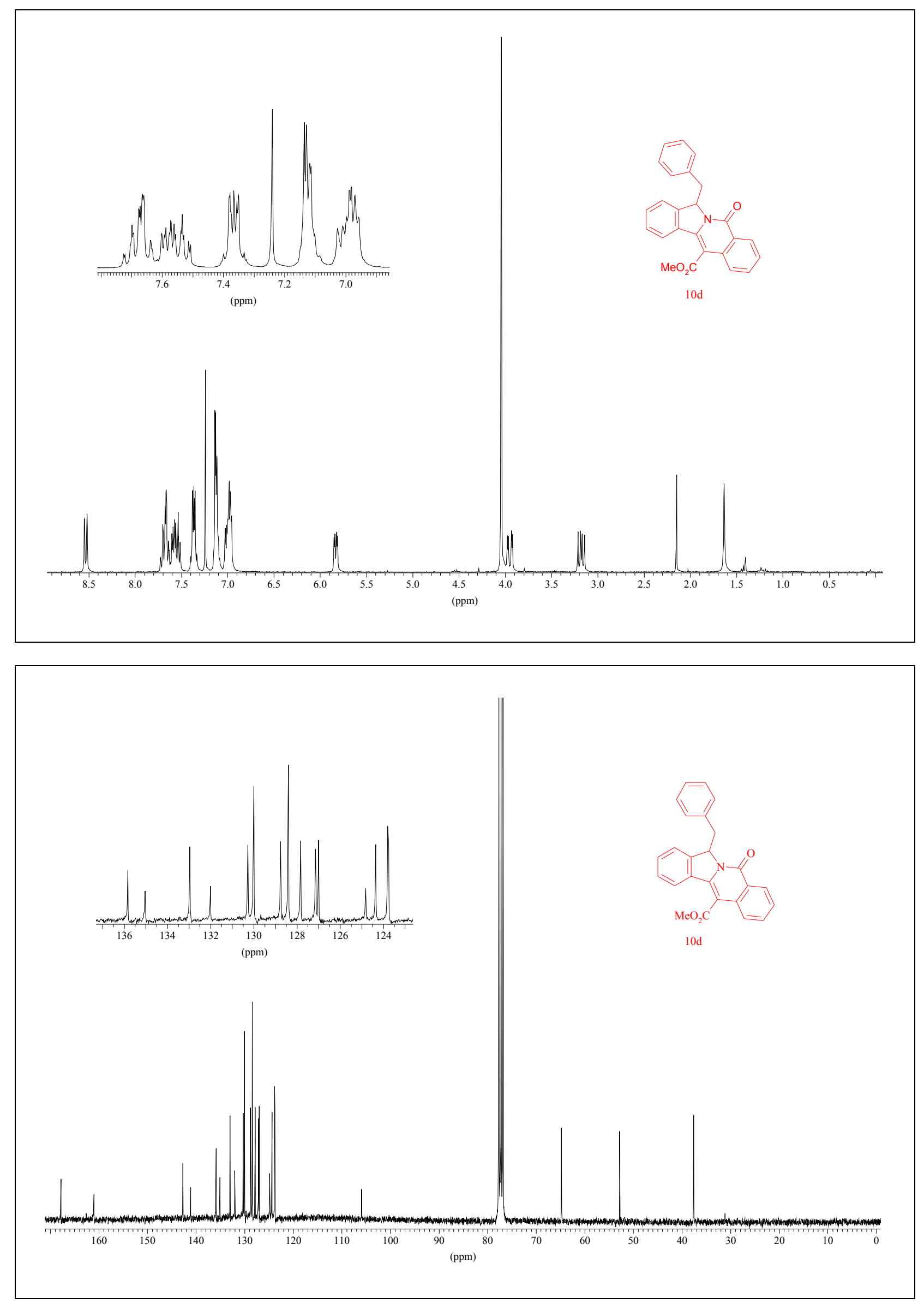

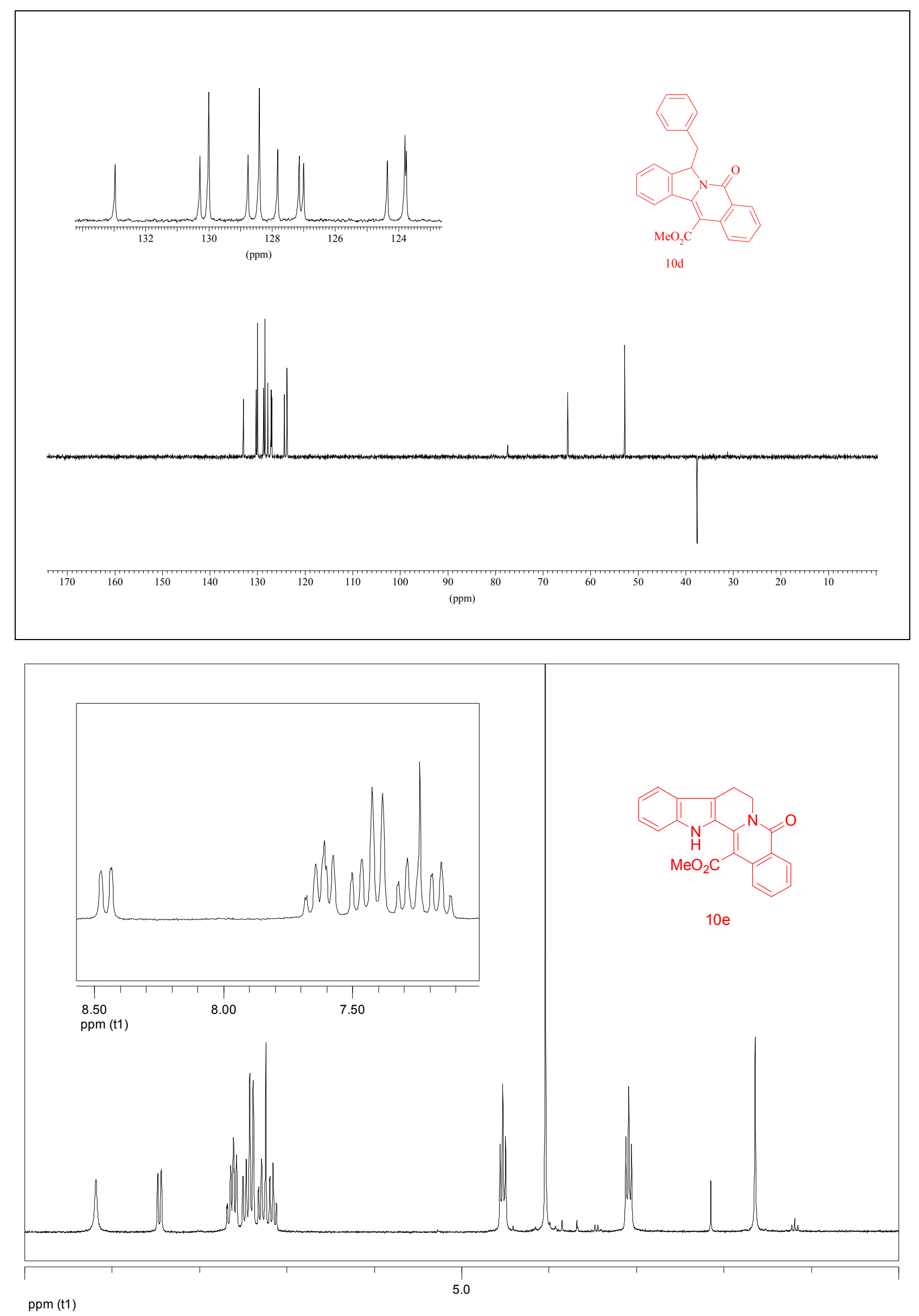


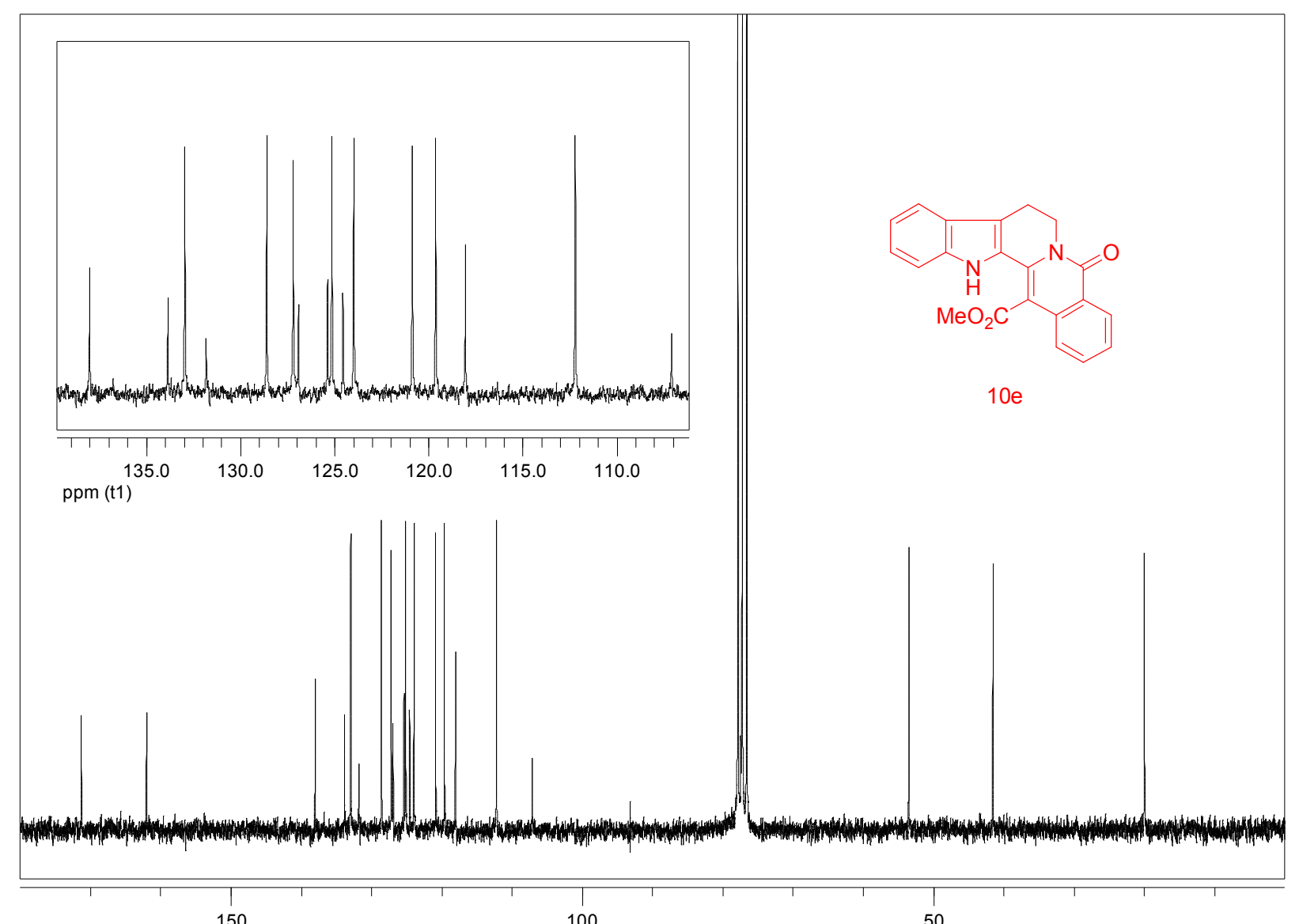

ppm (t1)

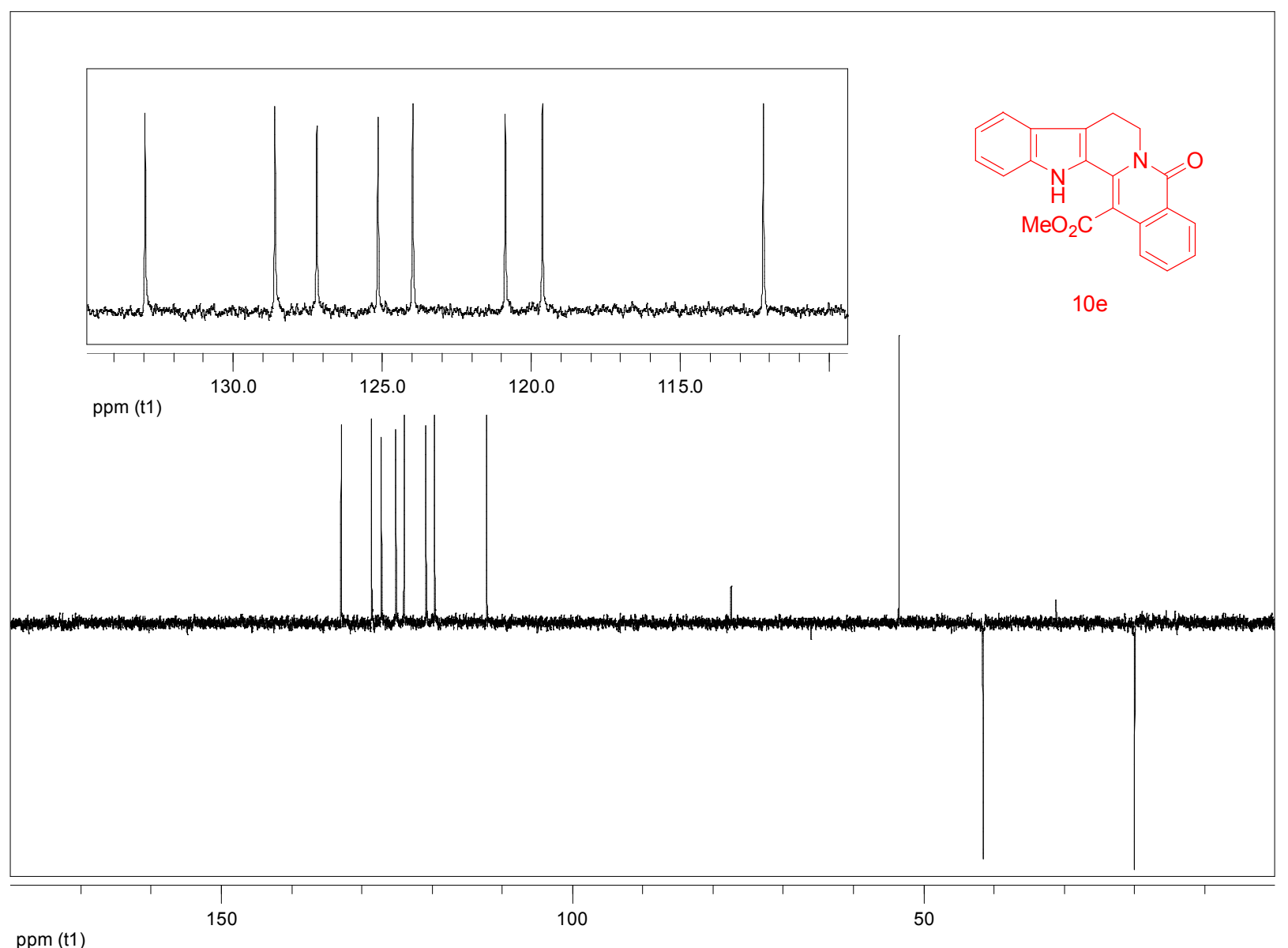

ppm (t1) 

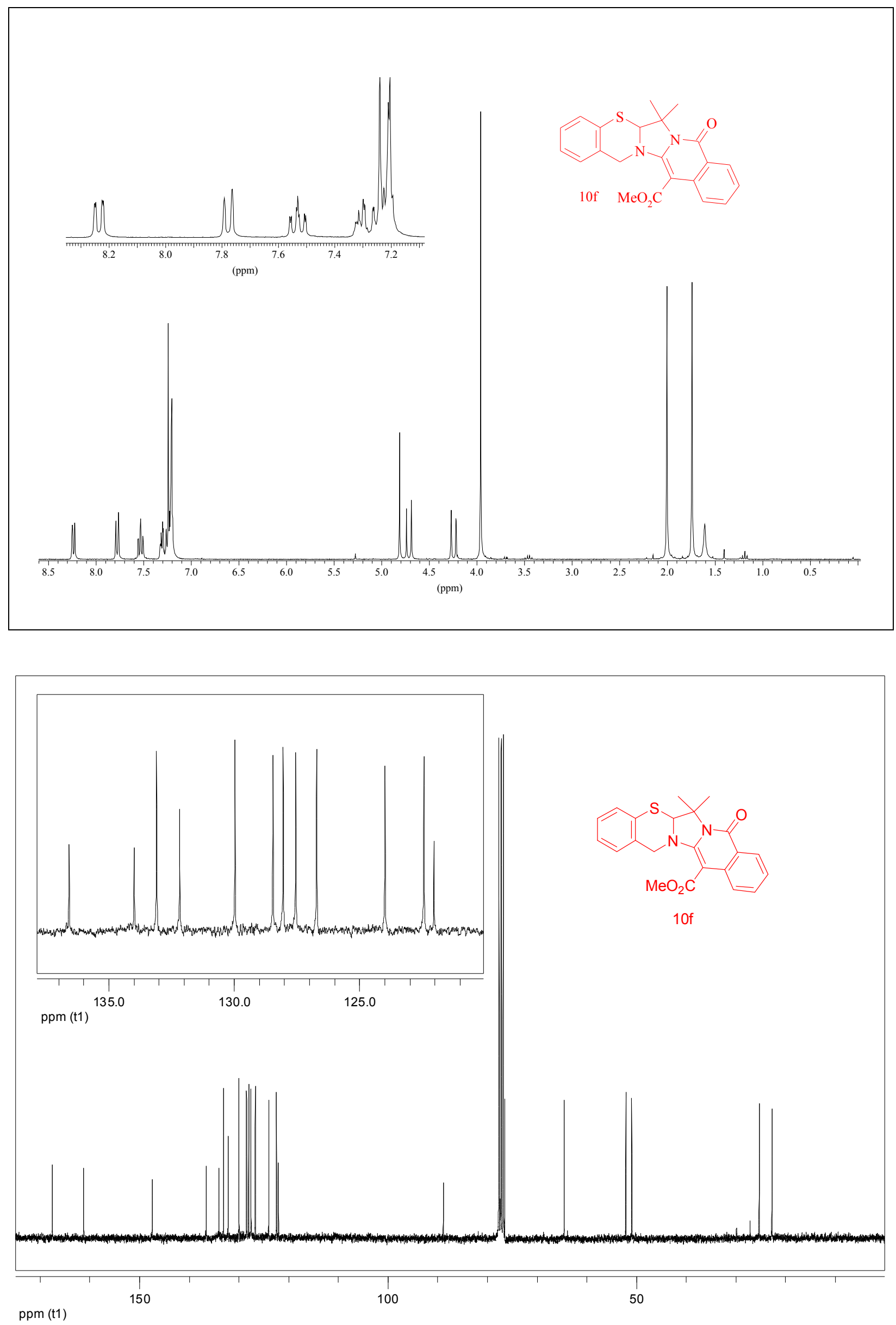


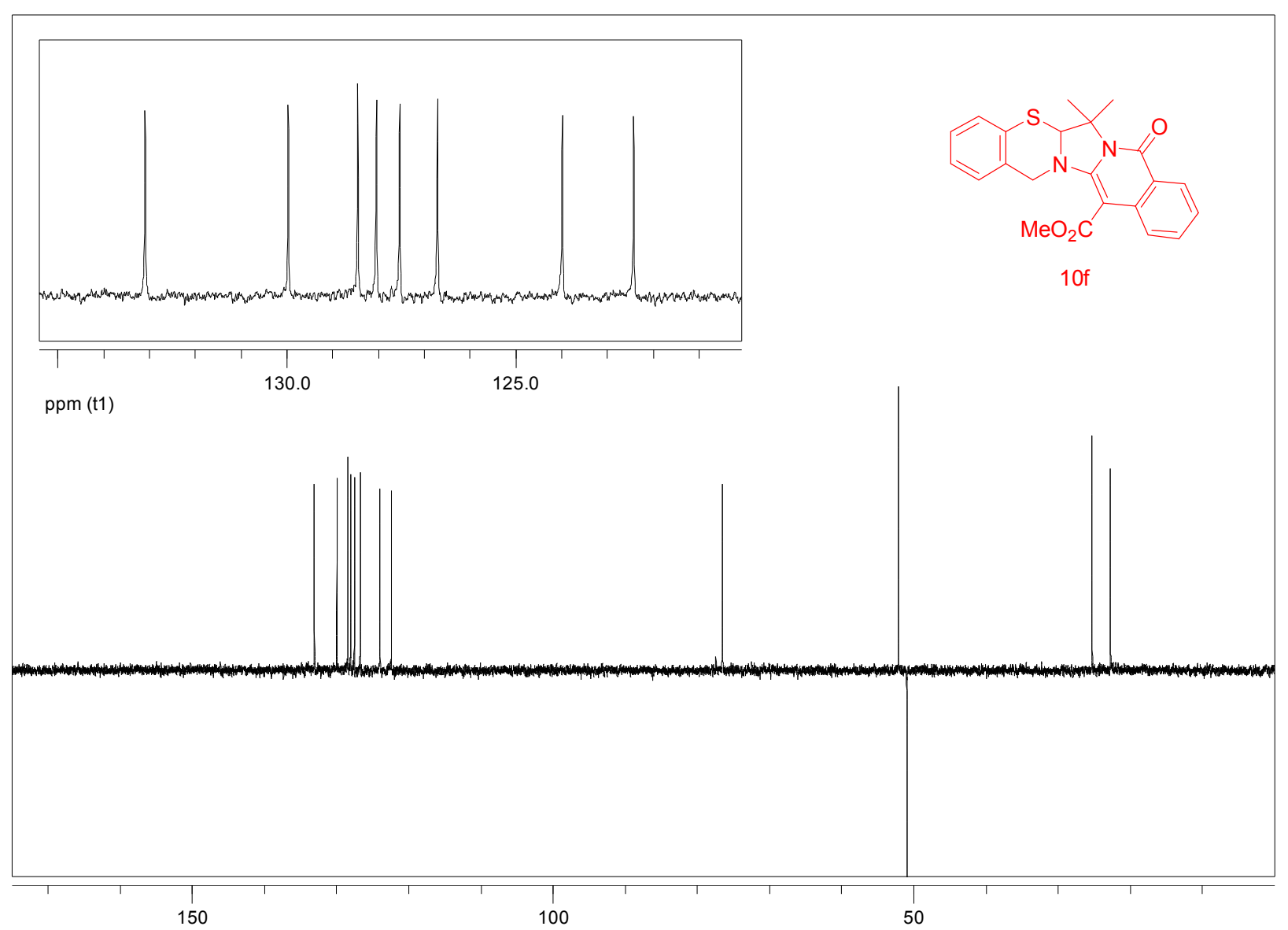

ppm (t1)

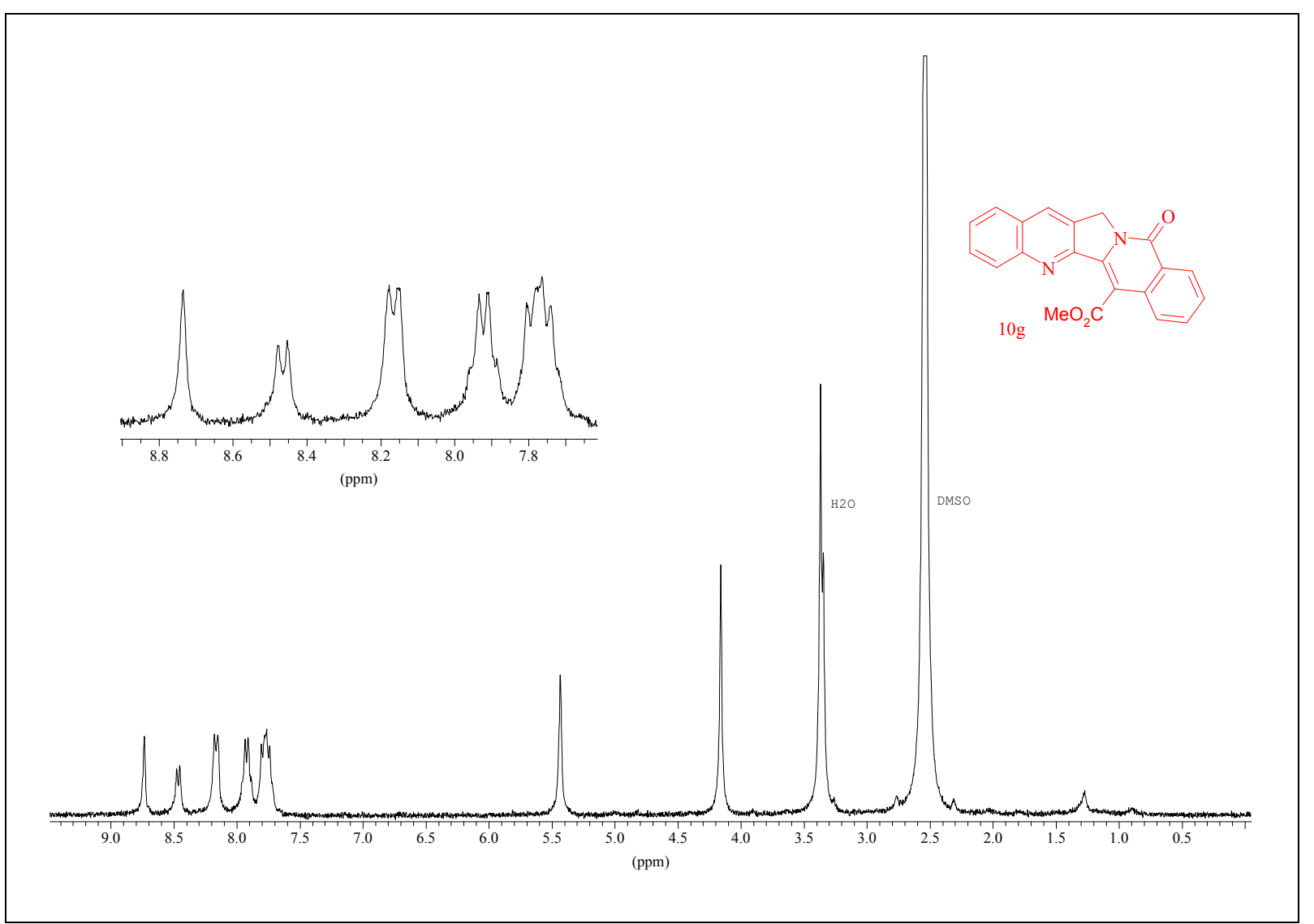




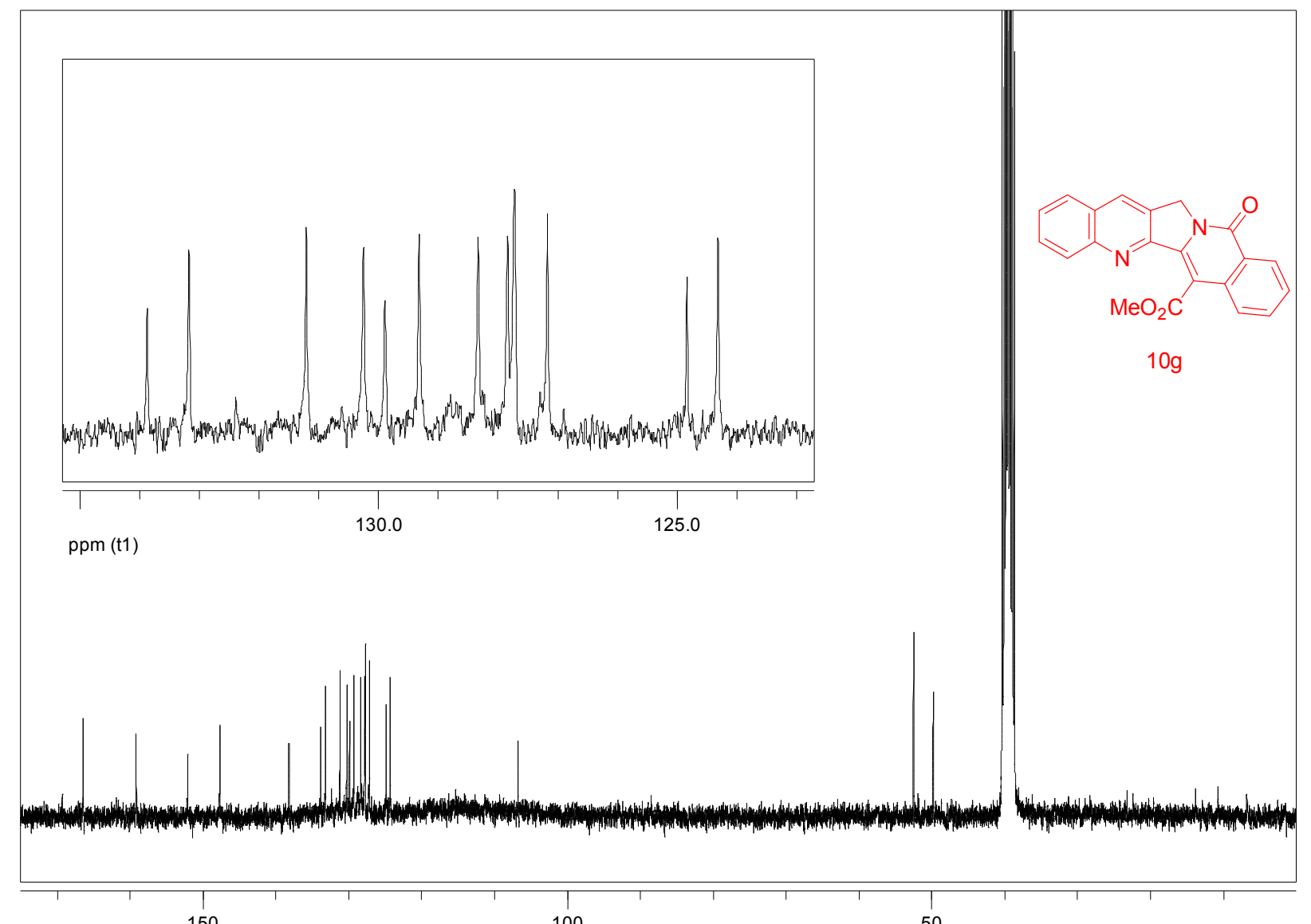

ppm (t1)

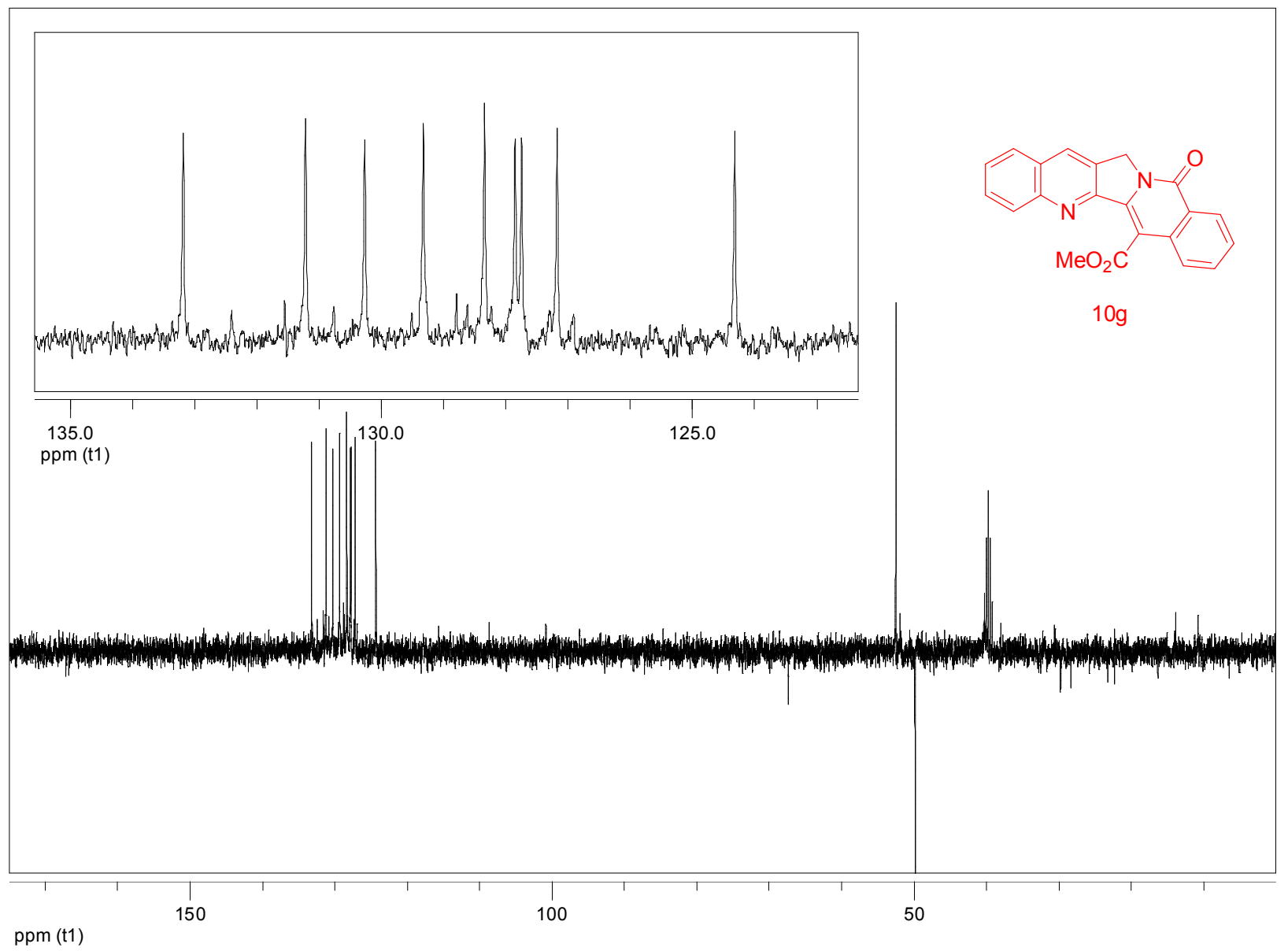




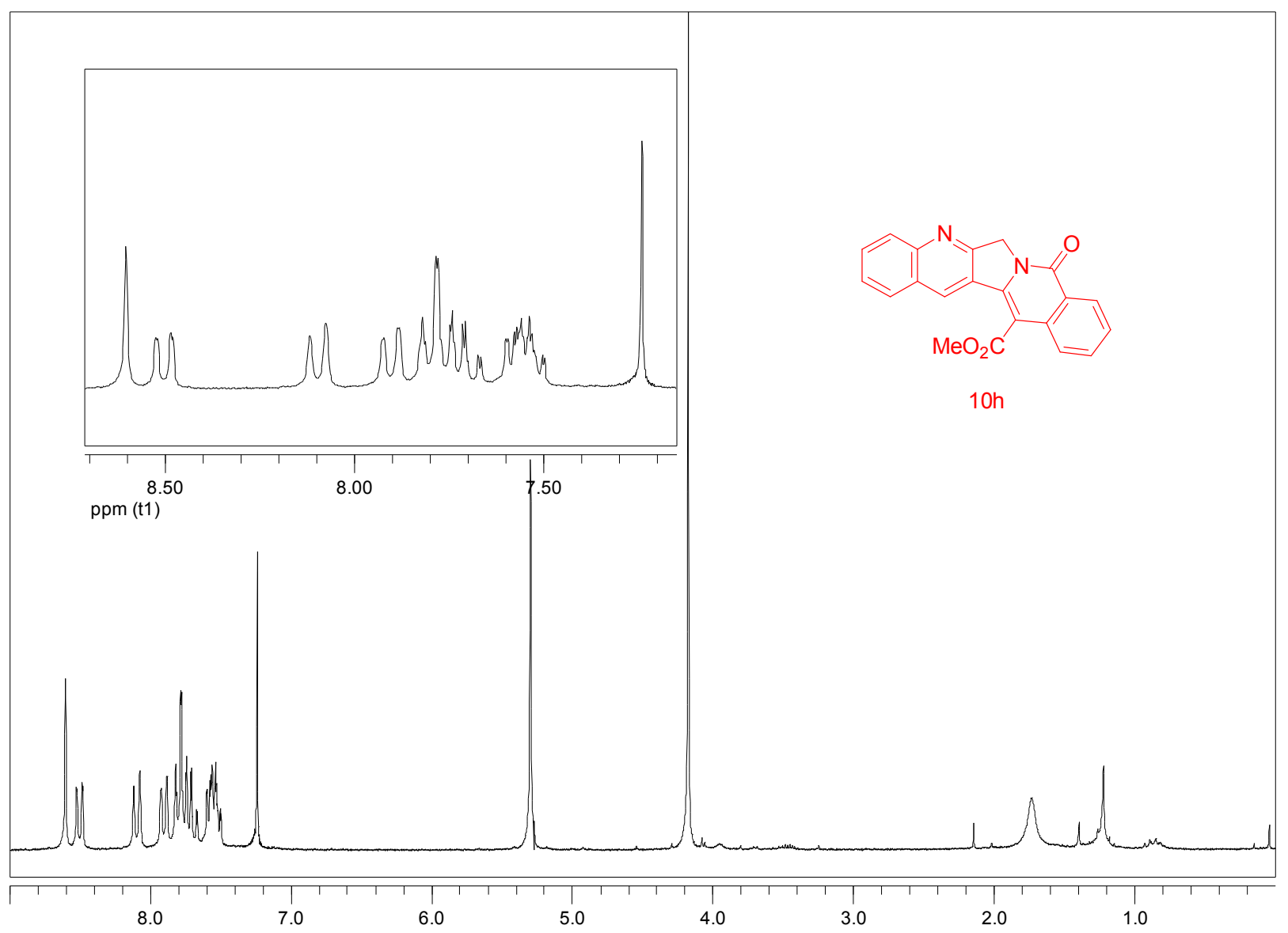

ppm (t1)

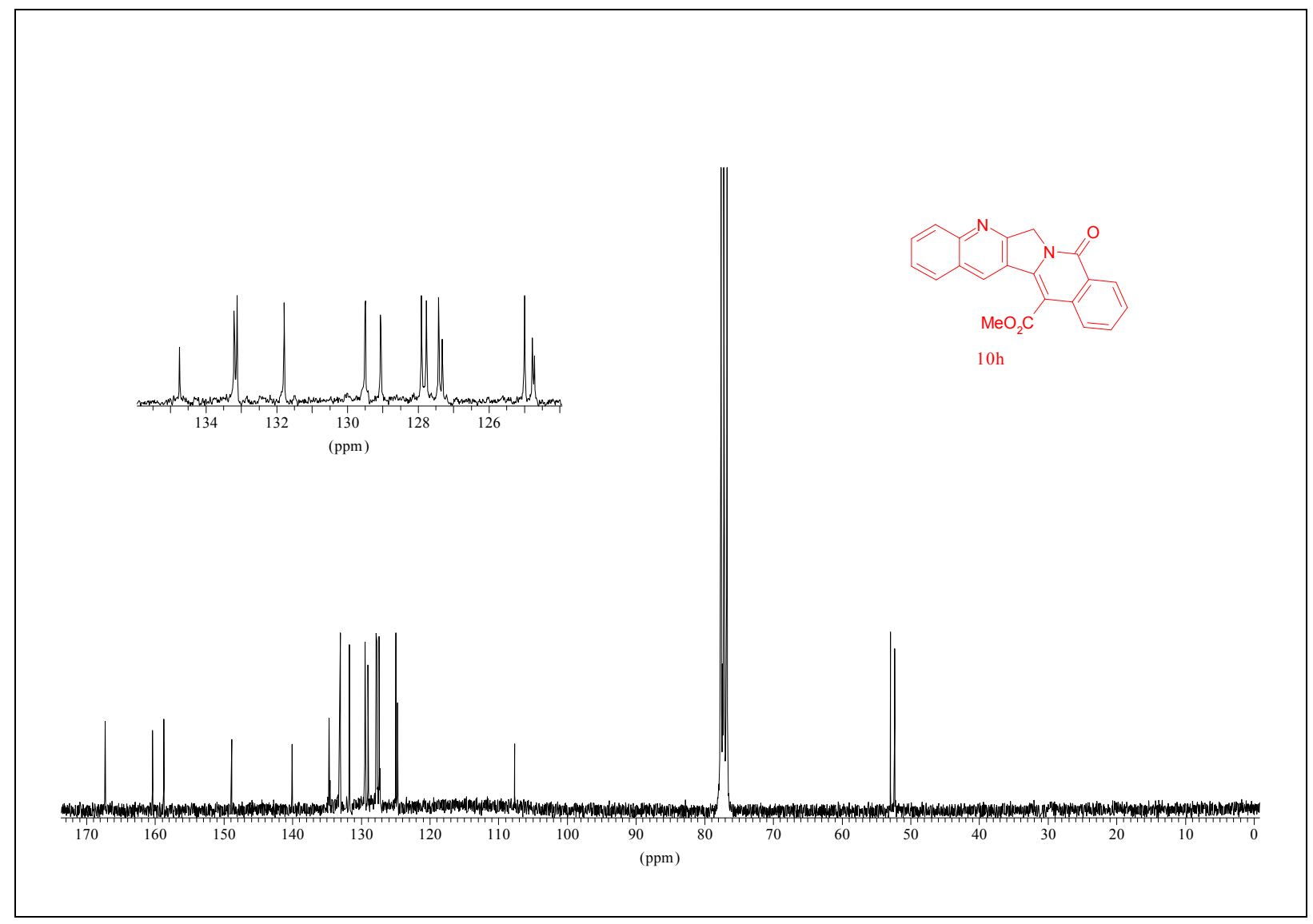



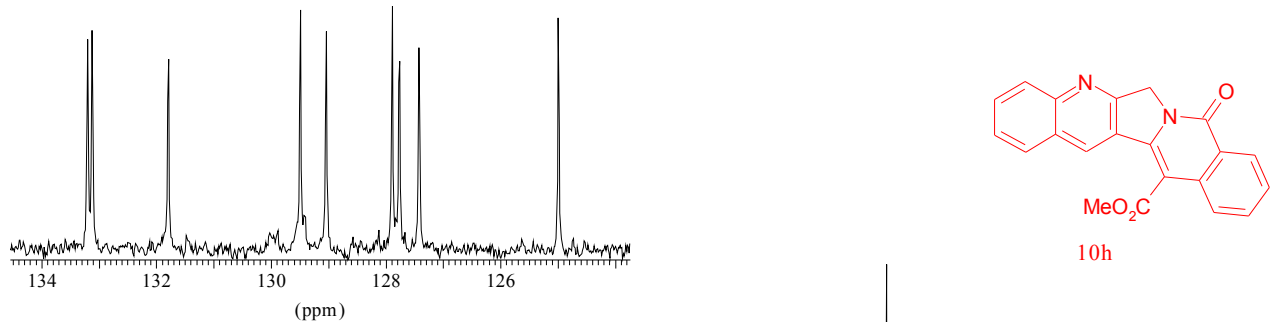

$10 \mathrm{~h}$

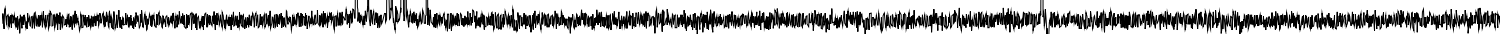

$\begin{array}{llllllllllllllll}170 & 160 & 150 & 140 & 130 & 120 & 110 & 100 & 90 & 80 & 70 & 60 & 50 & 40 & 30 & 20 \\ (\mathrm{ppm}) & & & & & \end{array}$

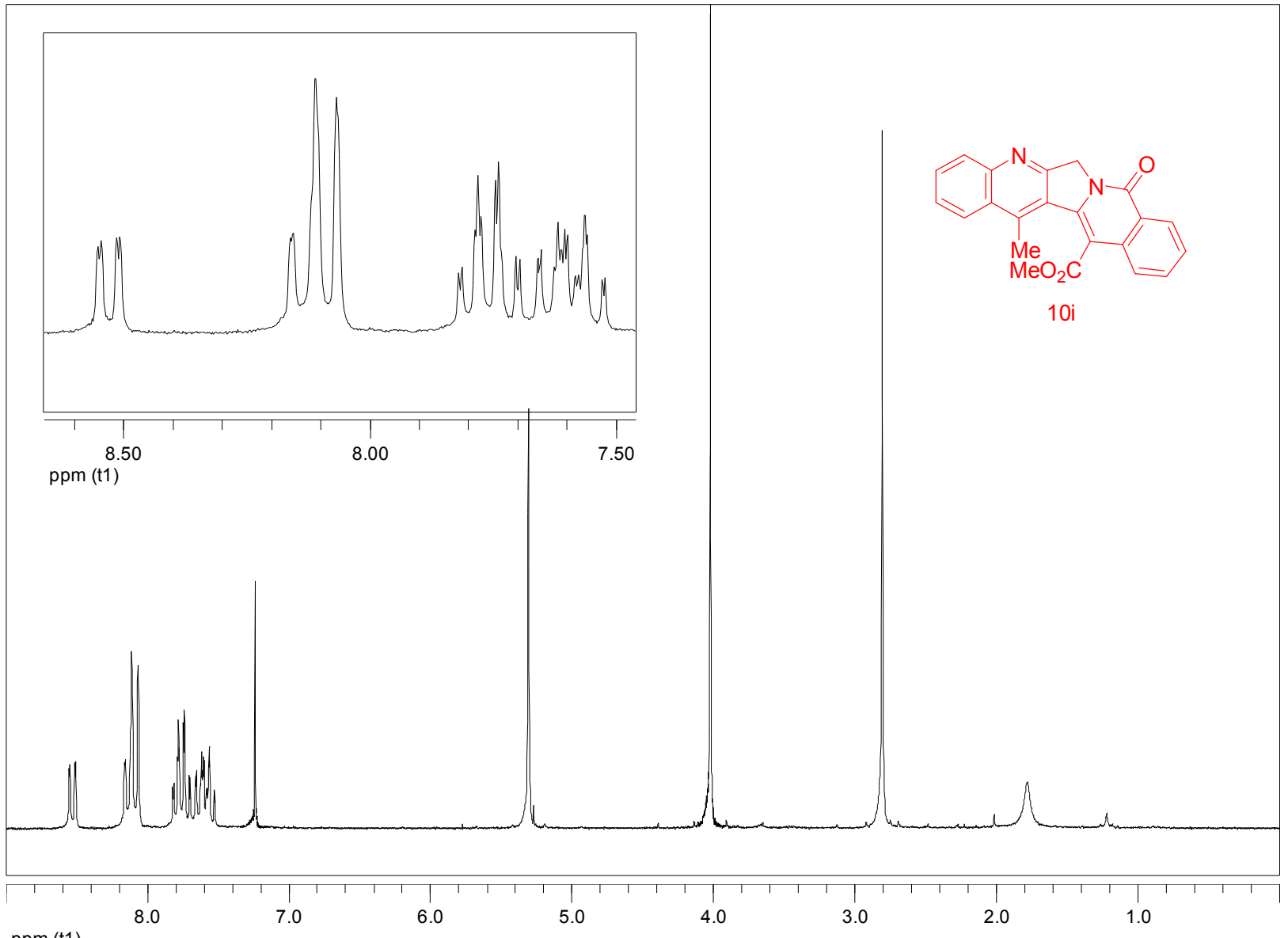

$\mathrm{ppm}(\mathrm{t} 1)$ 


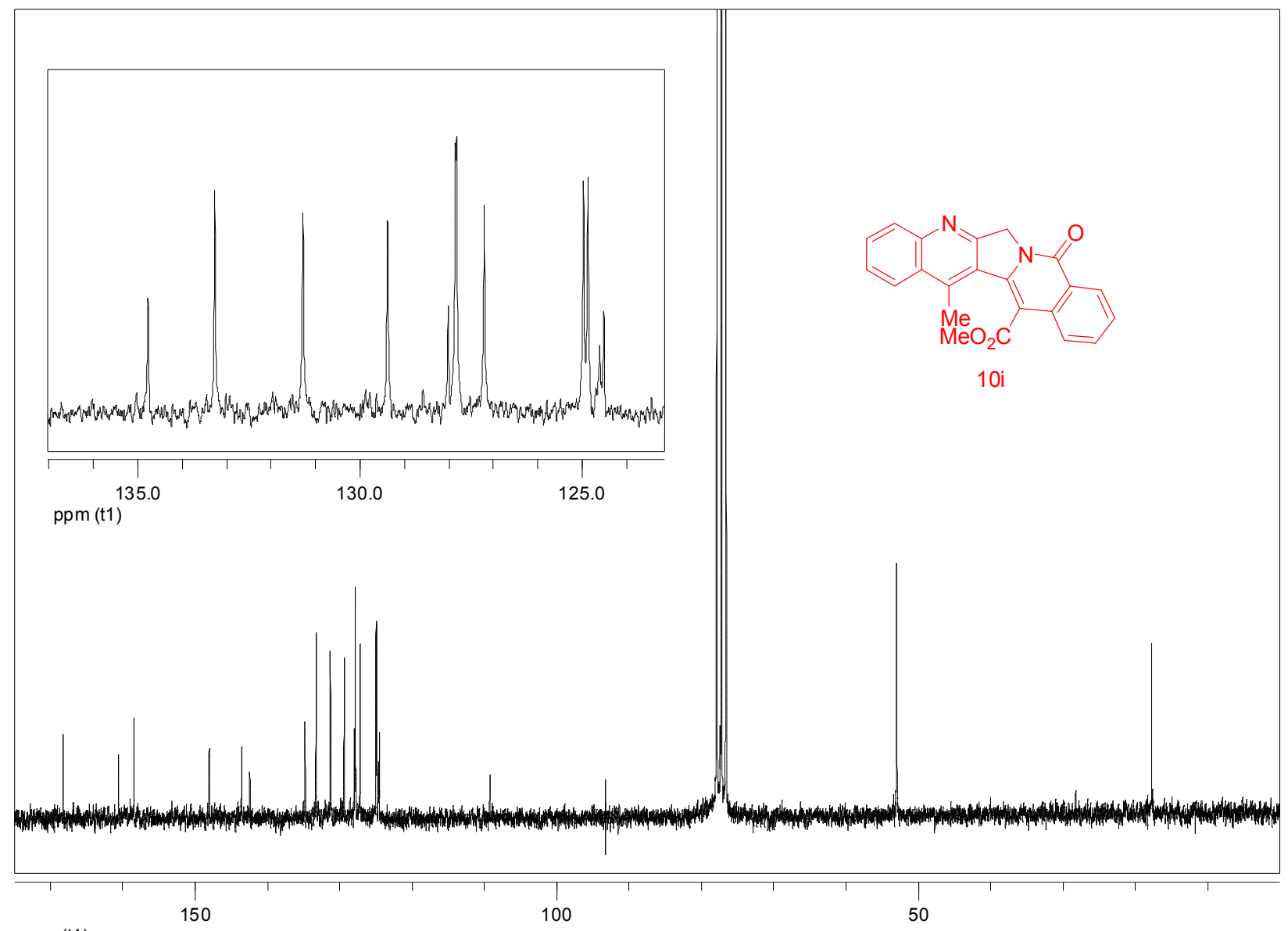

ppm (t1)
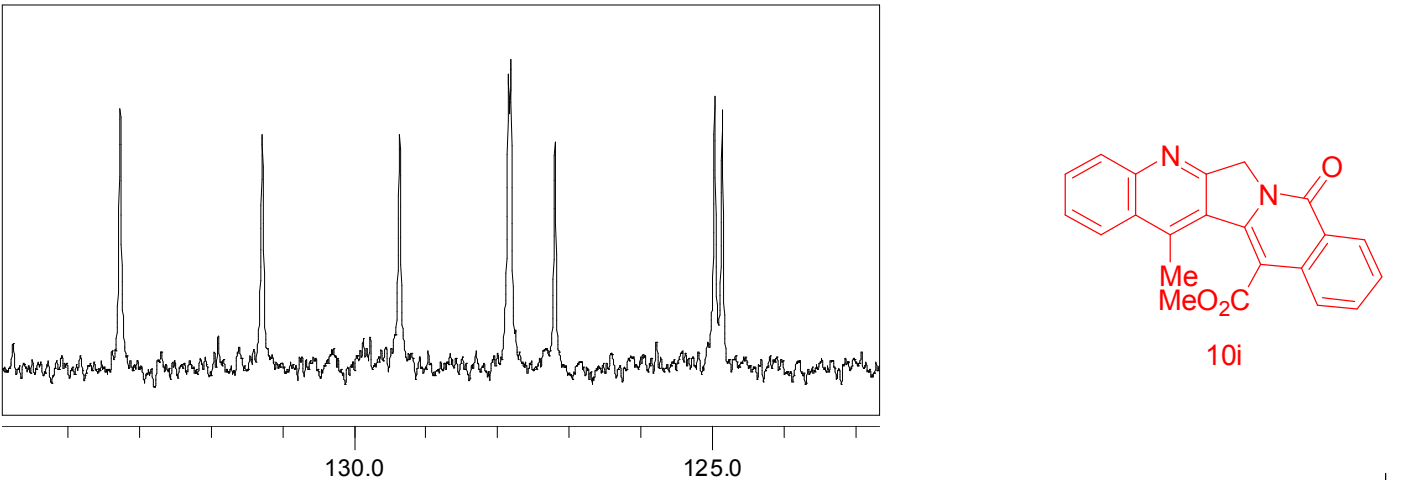

$10 \mathrm{i}$

ppm (t1)

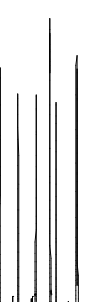

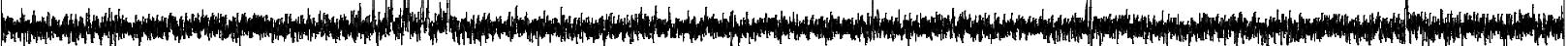

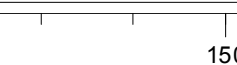

150

100

ppm (t1) 


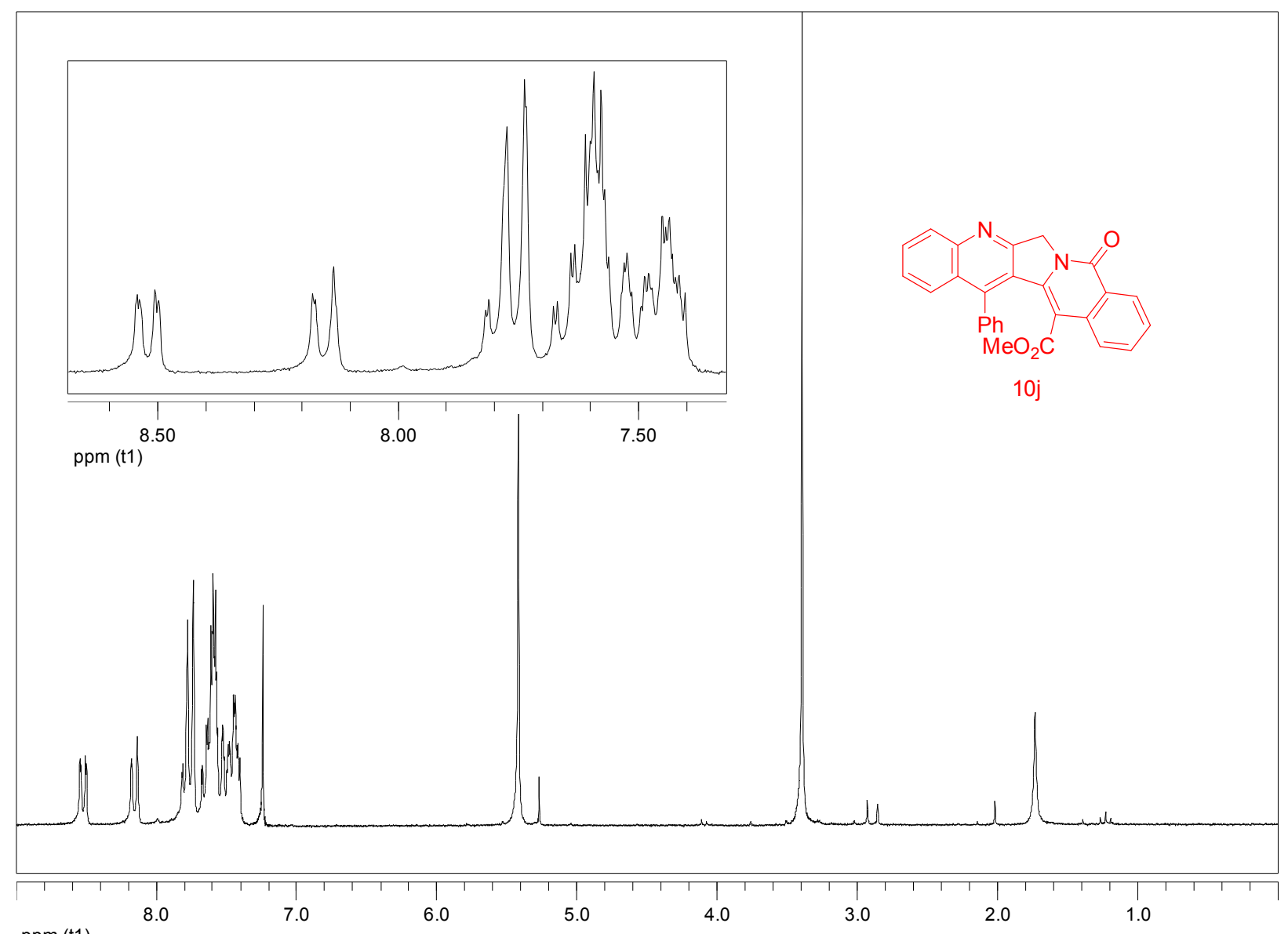

ppm (t1)

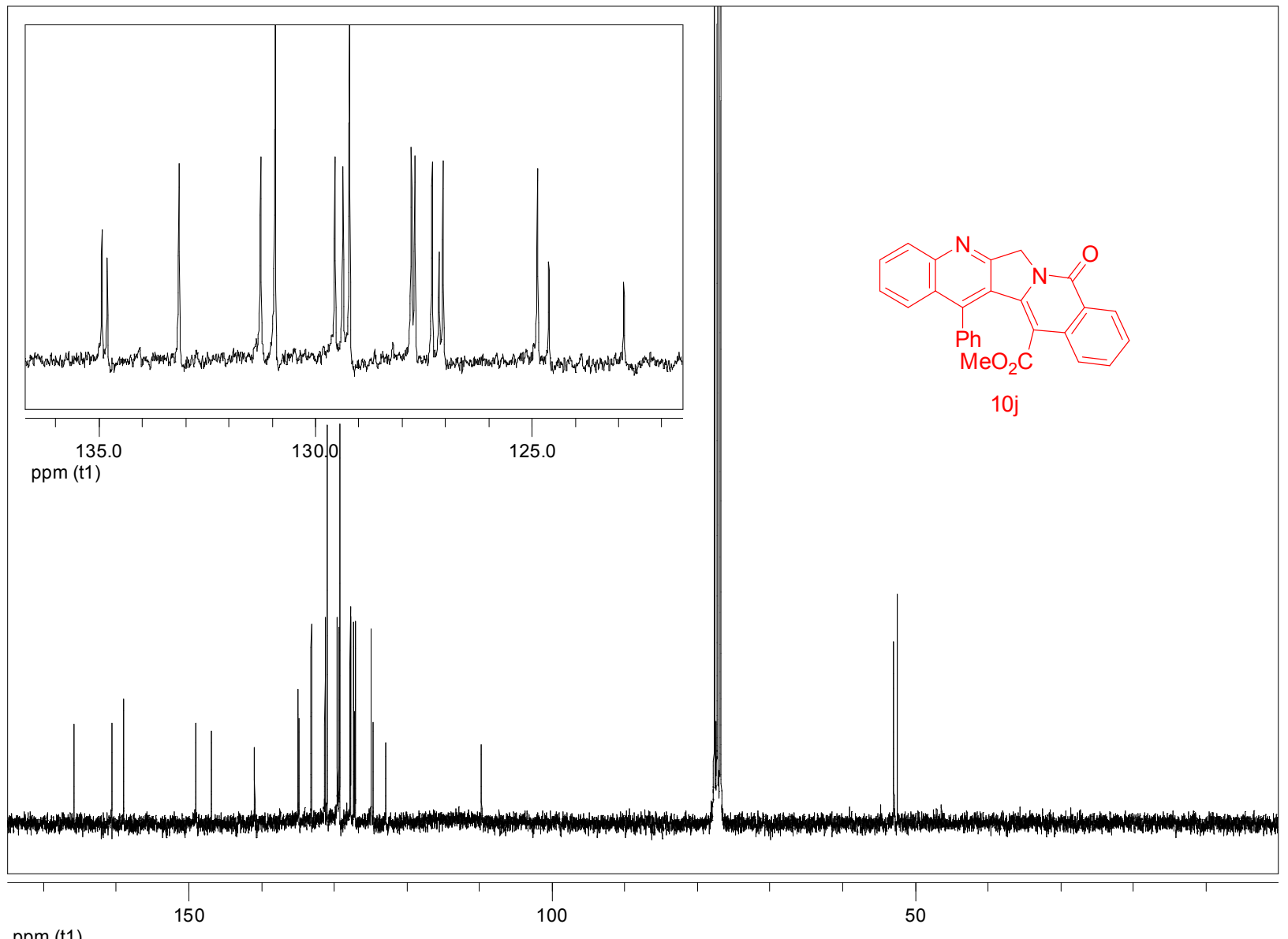




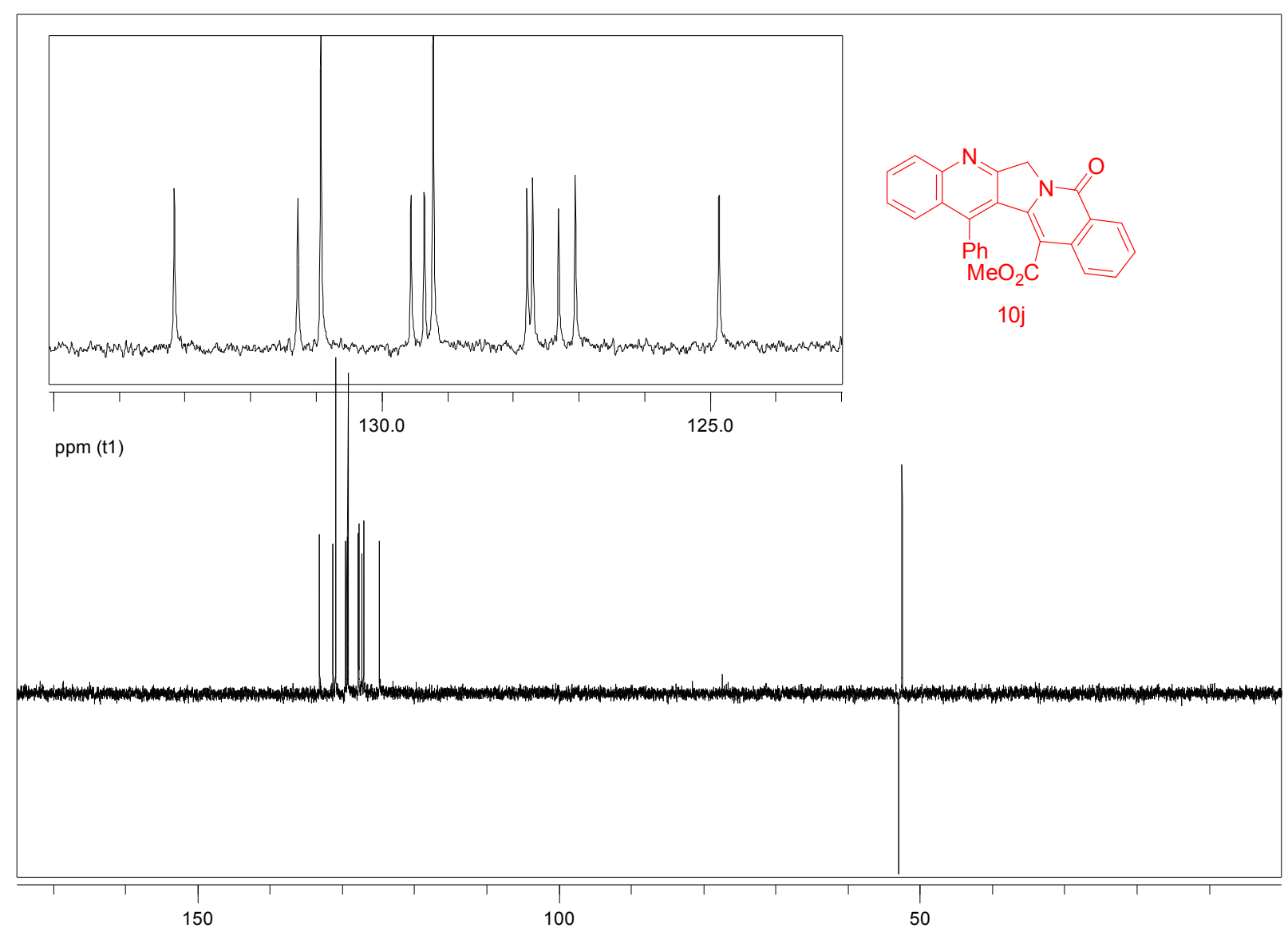

ppm (t1)

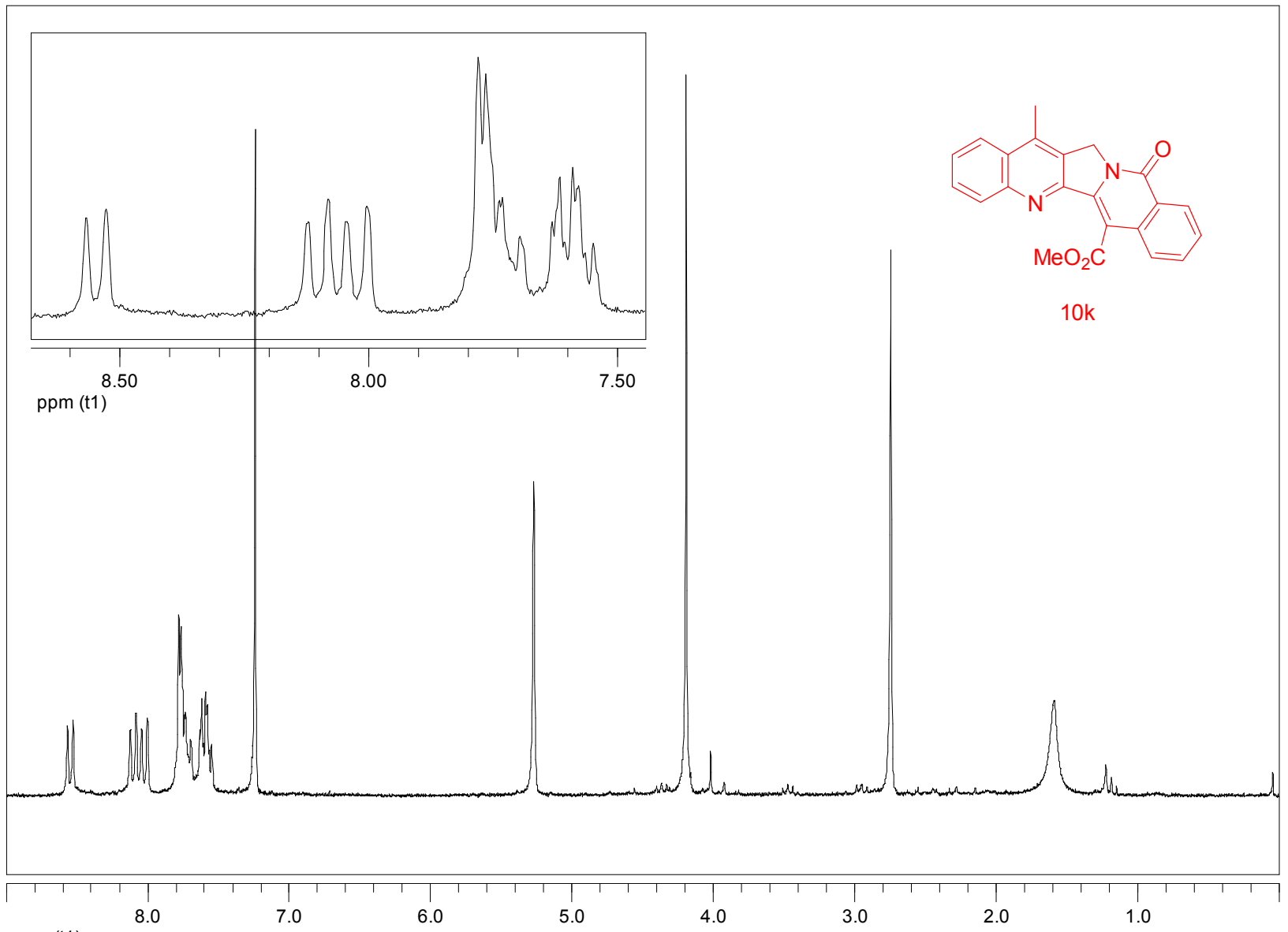

ppm (t1) 


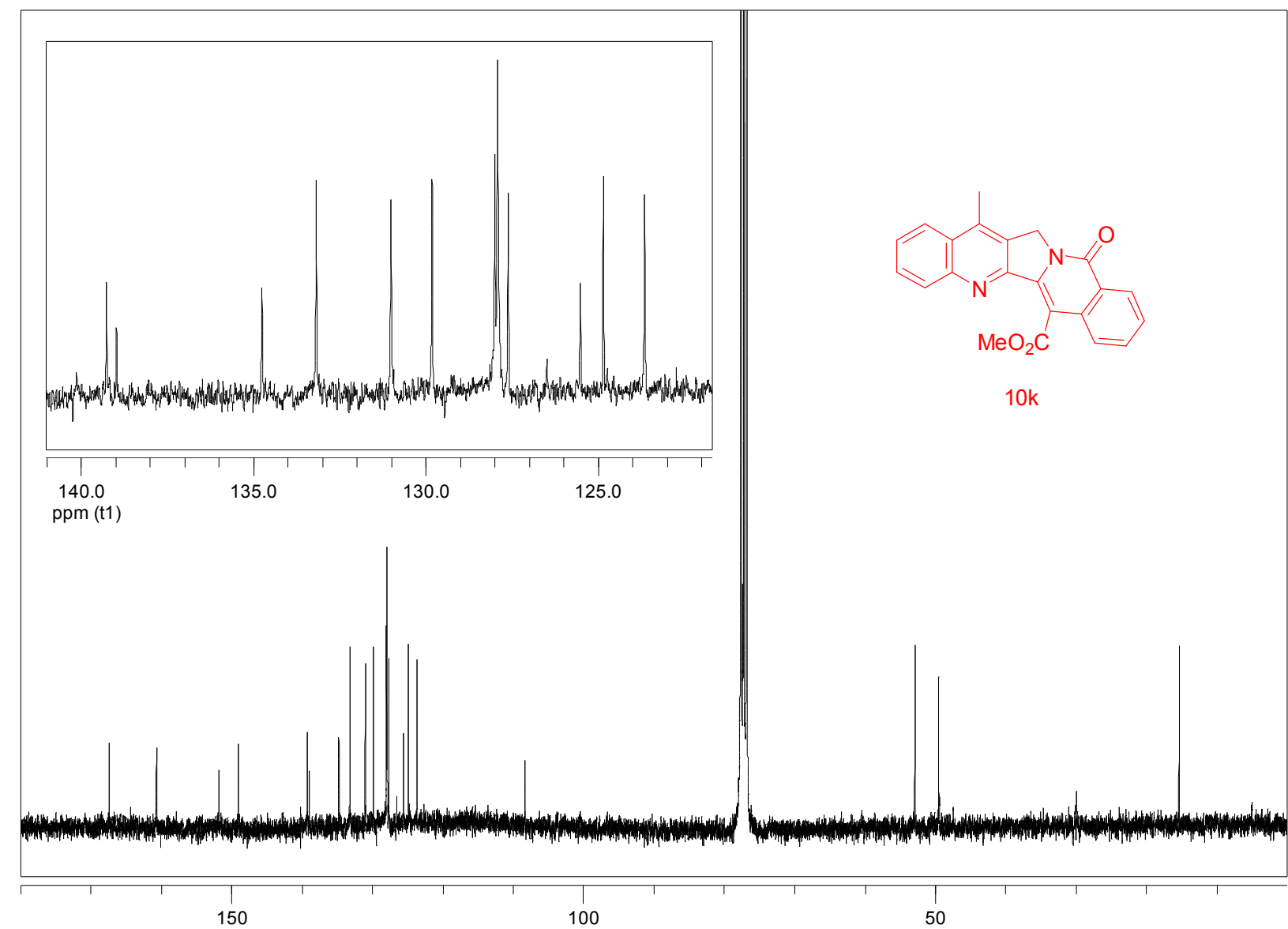

ppm (t1)

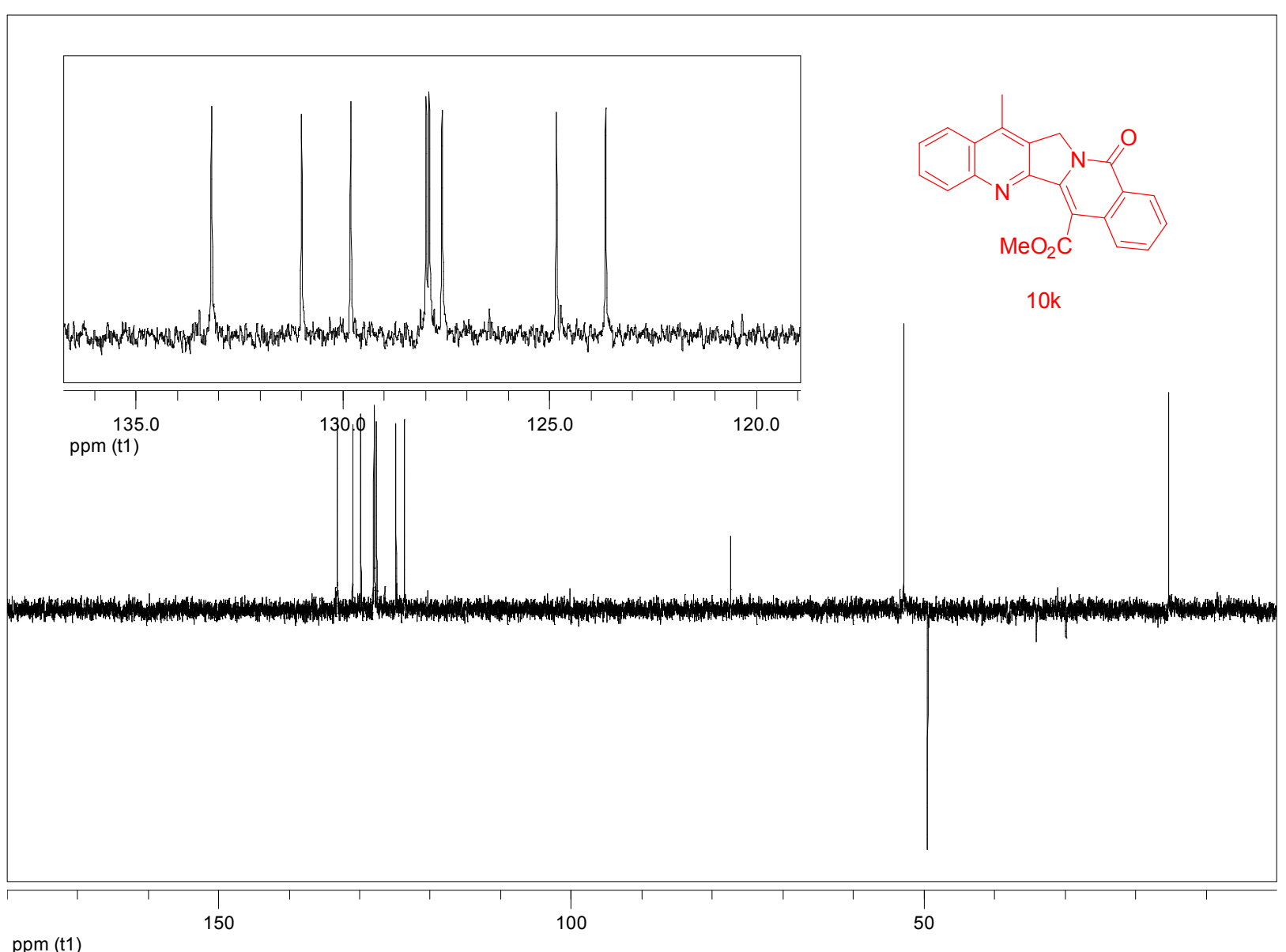




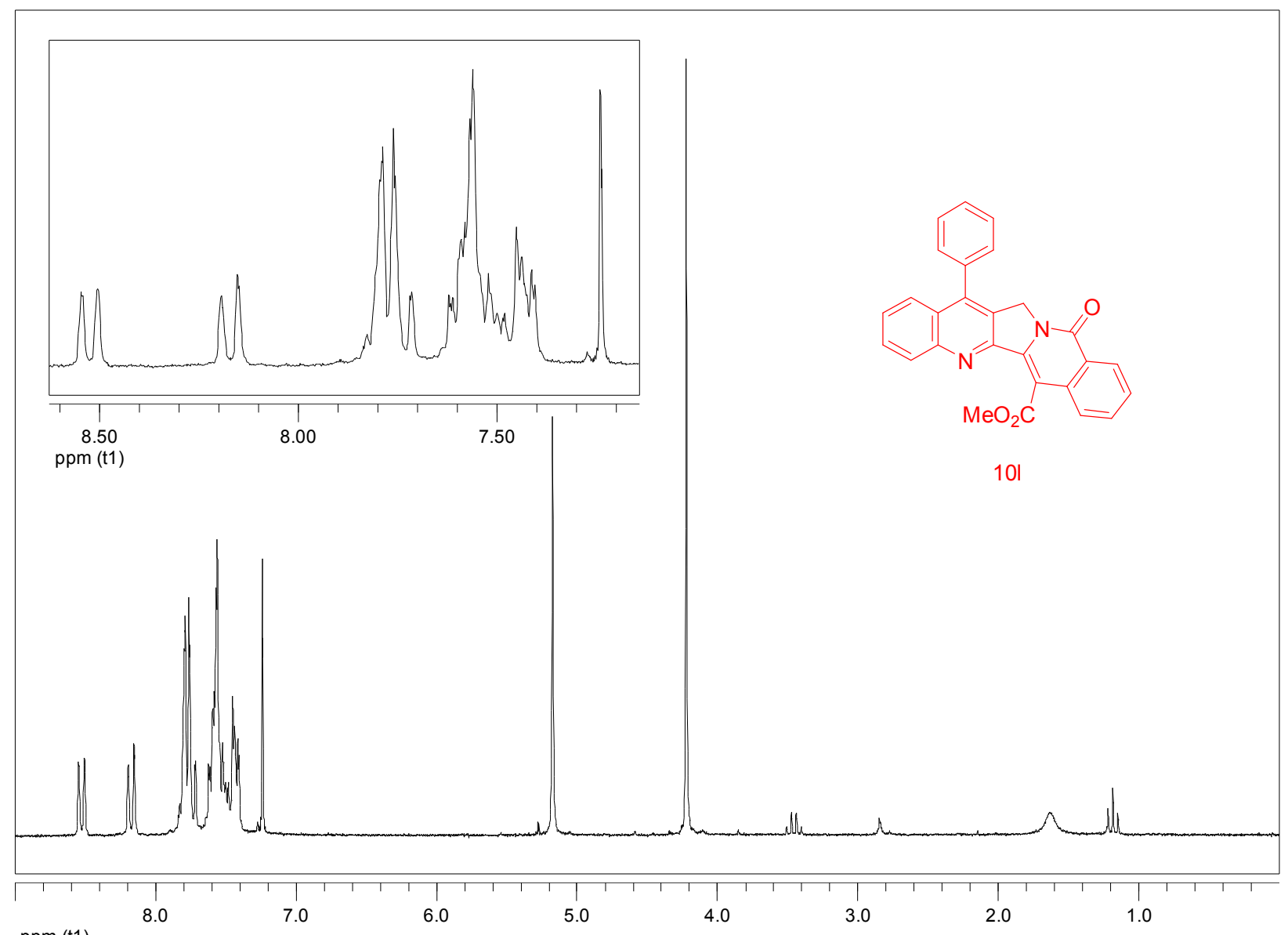

ppm (t1)

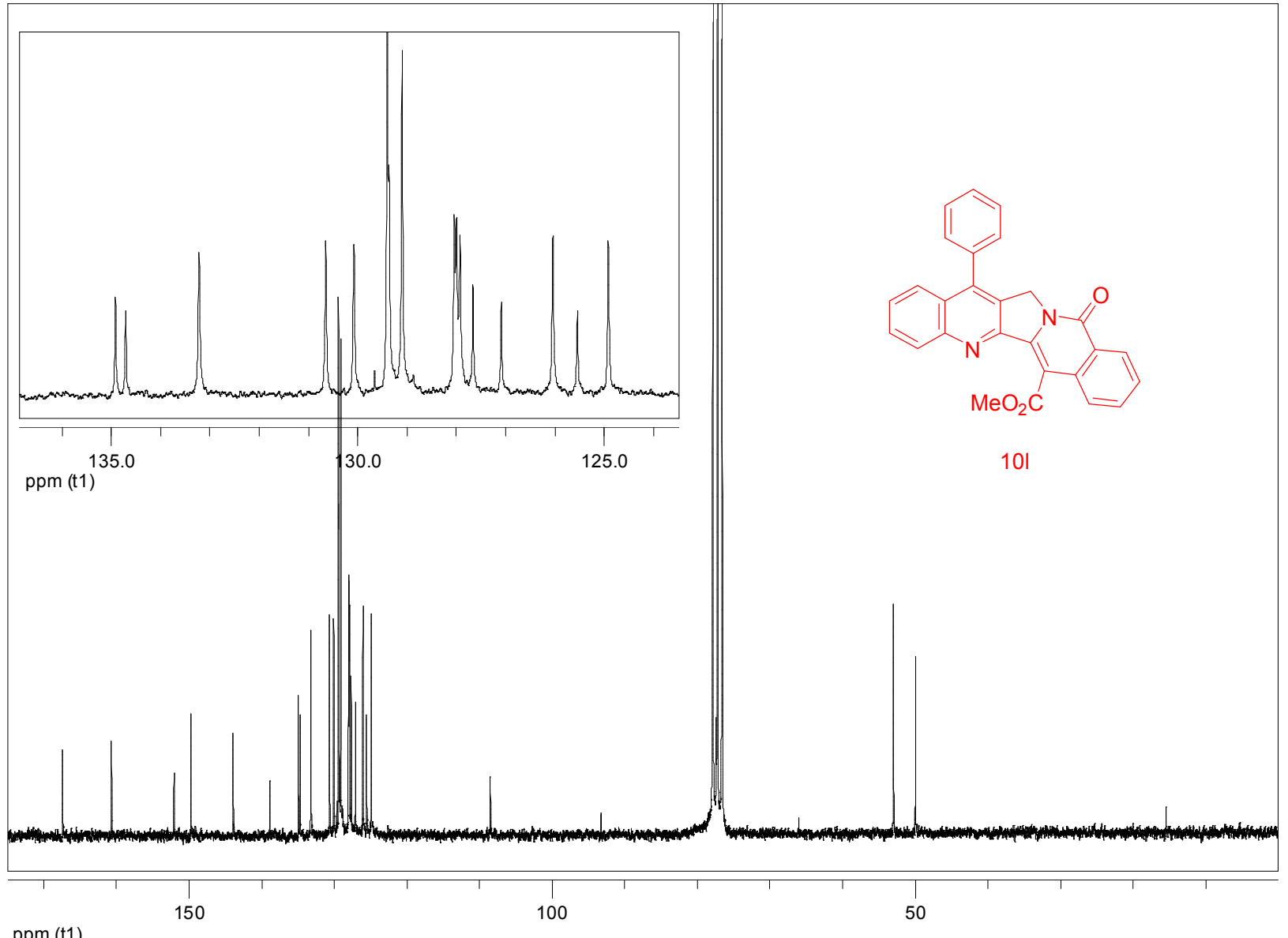




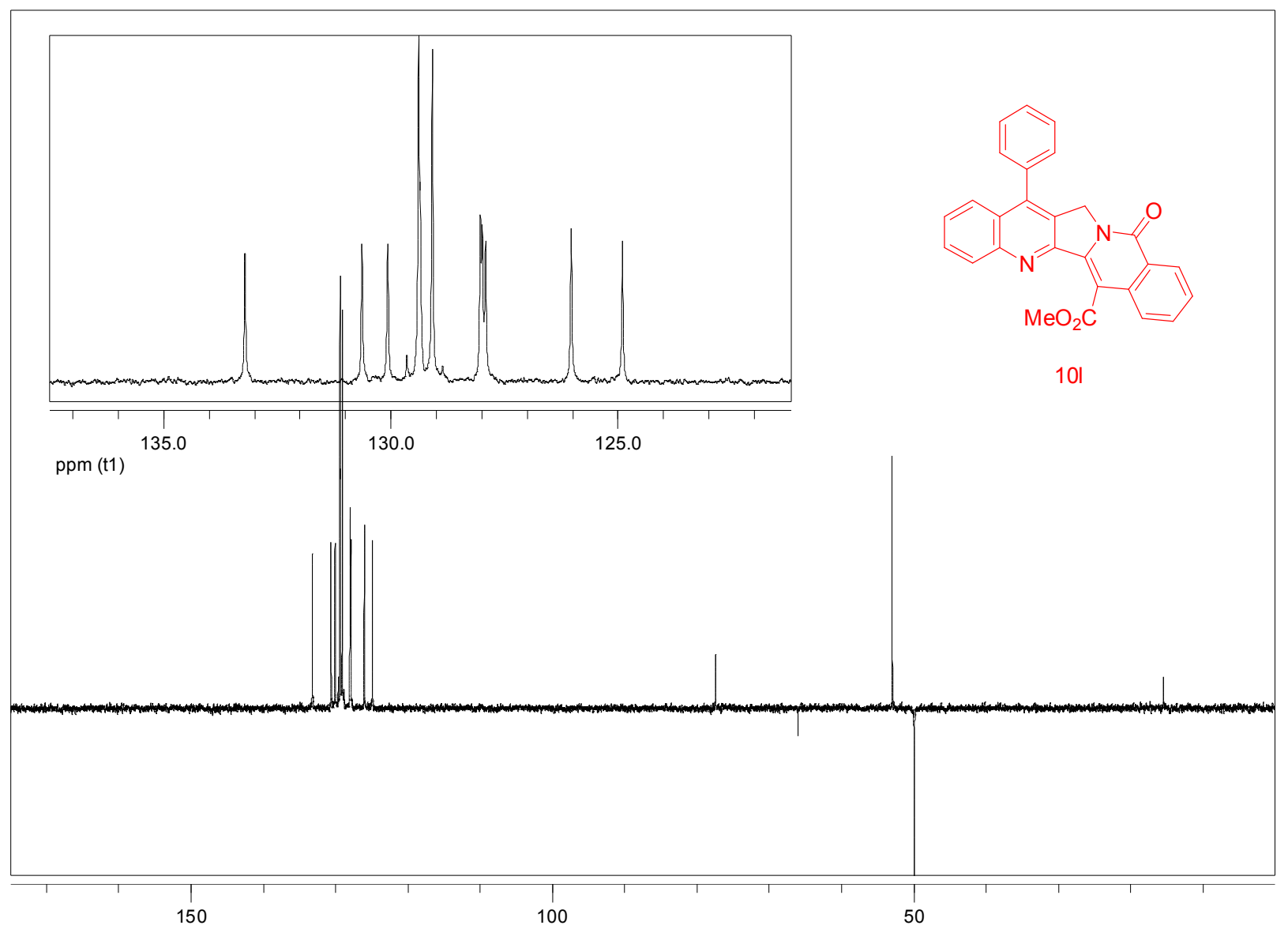

ppm (t1)

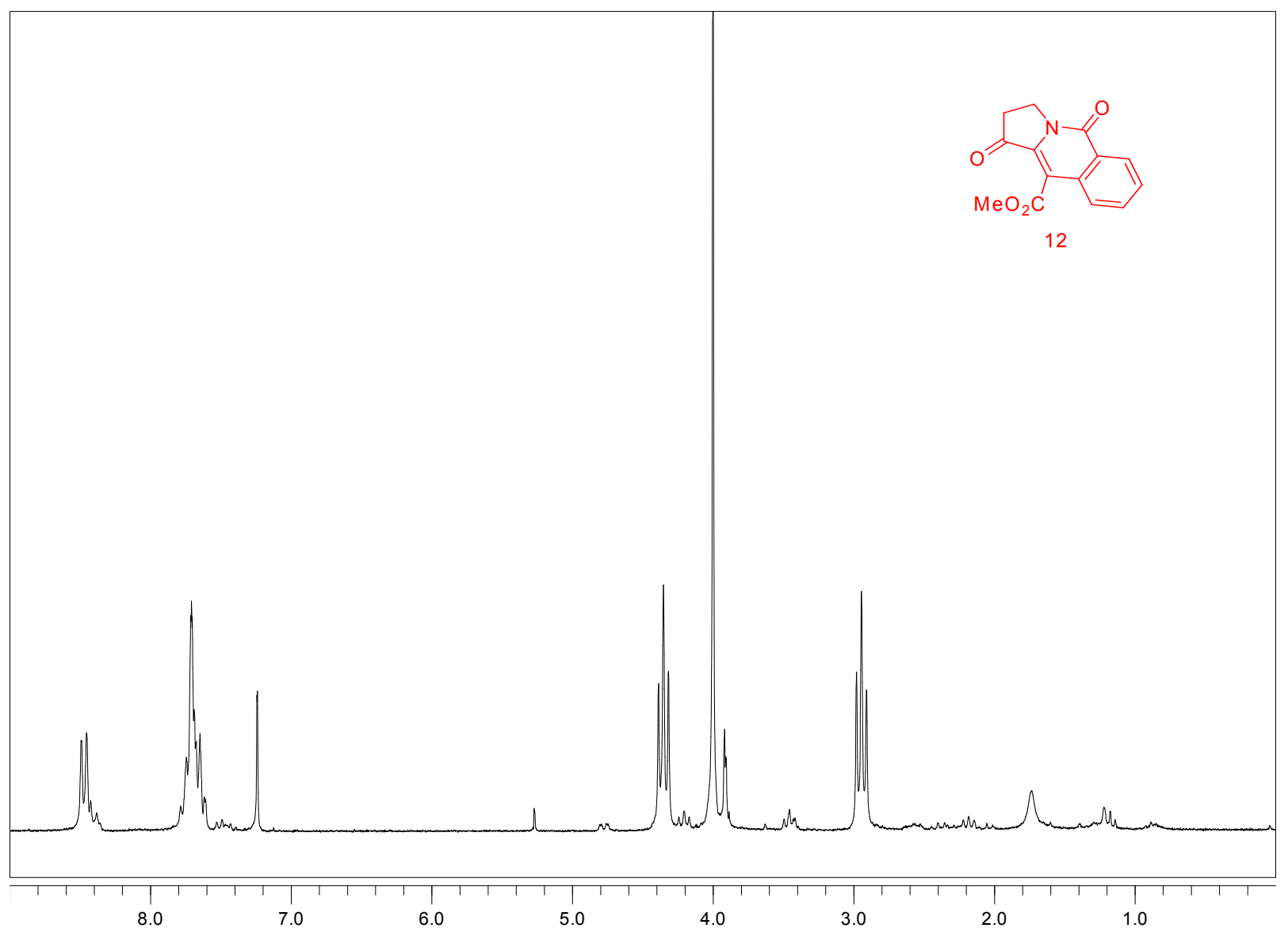

ppm (t1) 

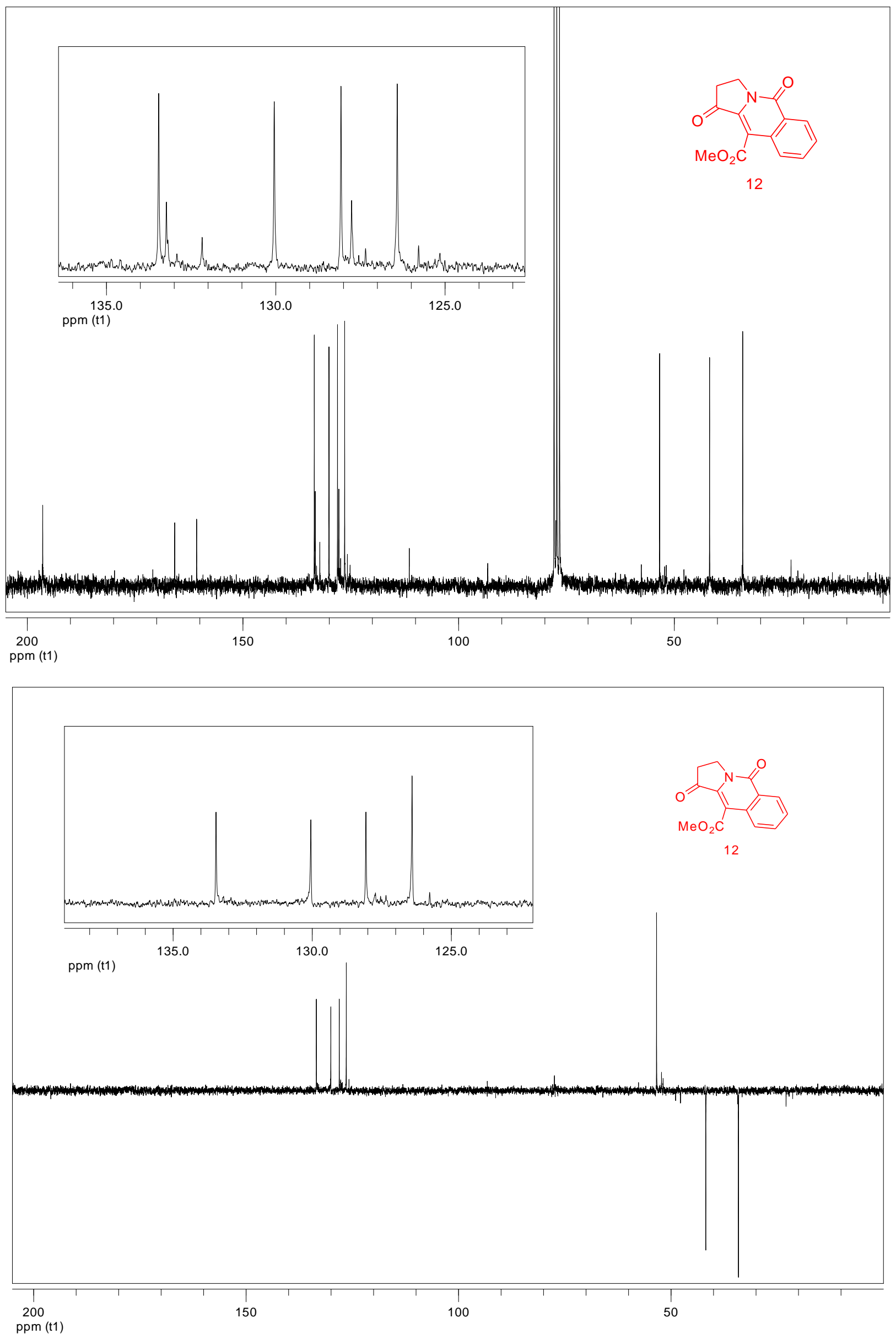
Proton $\mathrm{CDCl} 3$

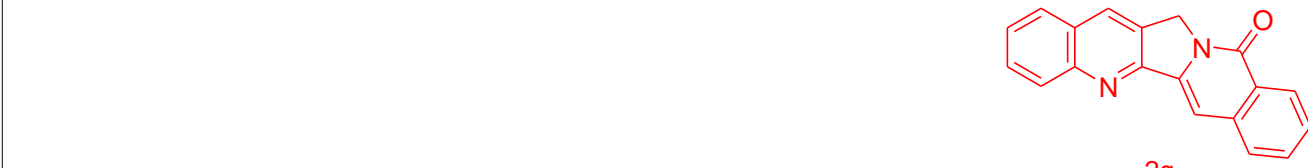

$2 g$

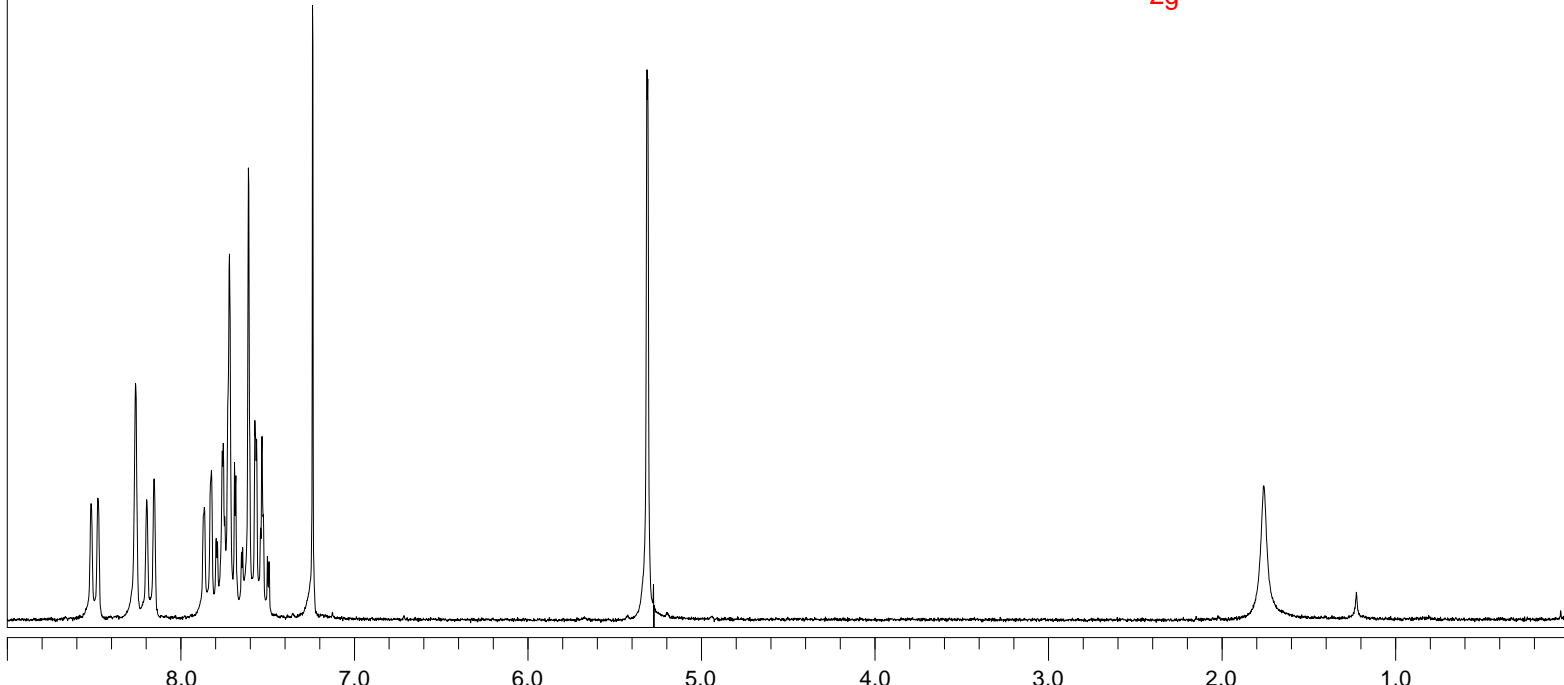

ppm (t1)

$\mathrm{C} 13 \mathrm{CDCl} 3$

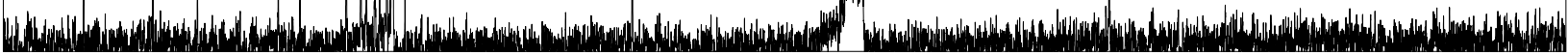

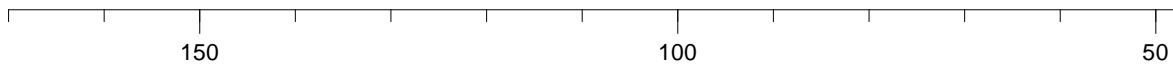

ppm (t1)

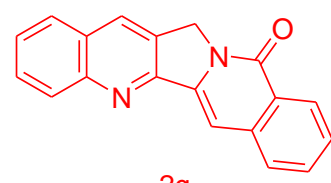

$2 g$ 
Dept $135 \mathrm{CDCl} 3$

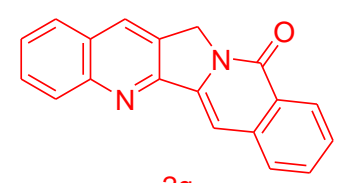

$2 g$

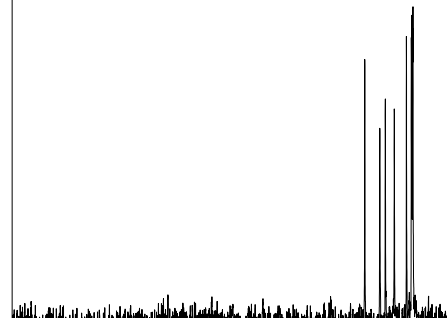

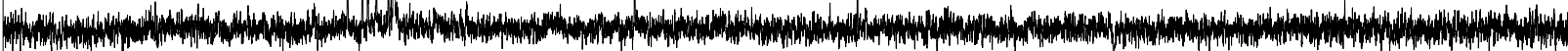

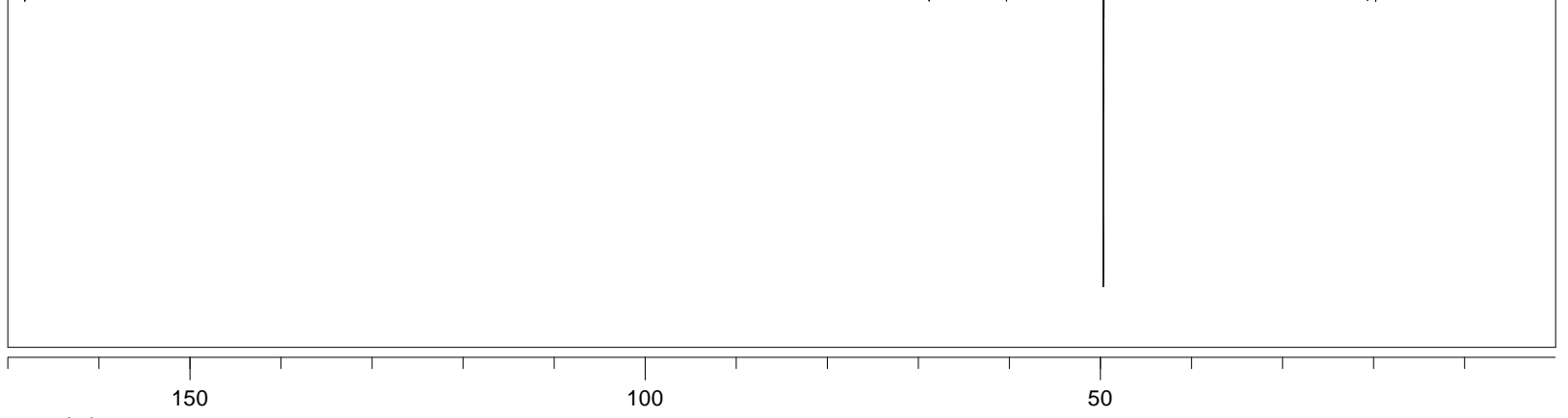

ppm (t1)

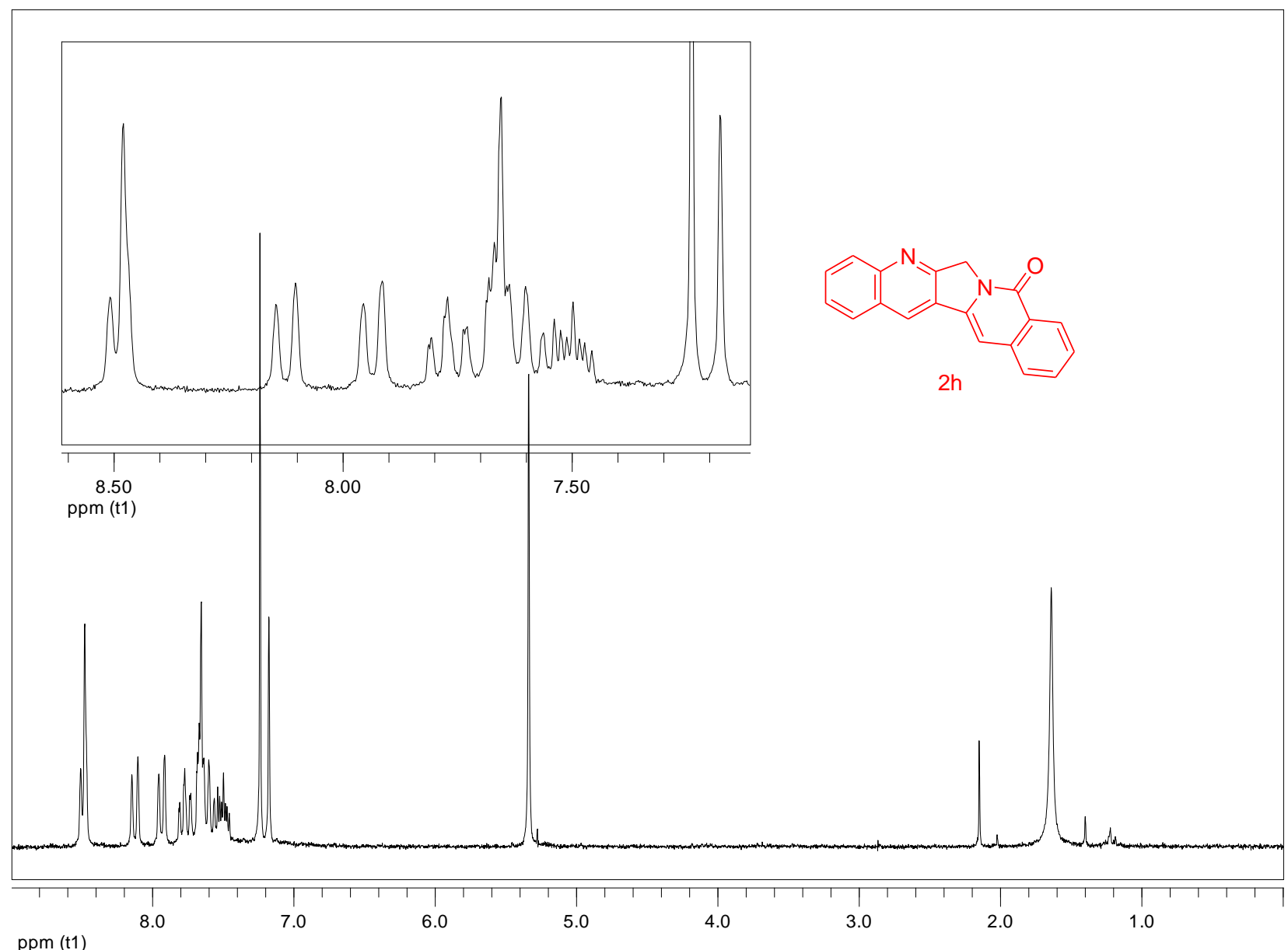



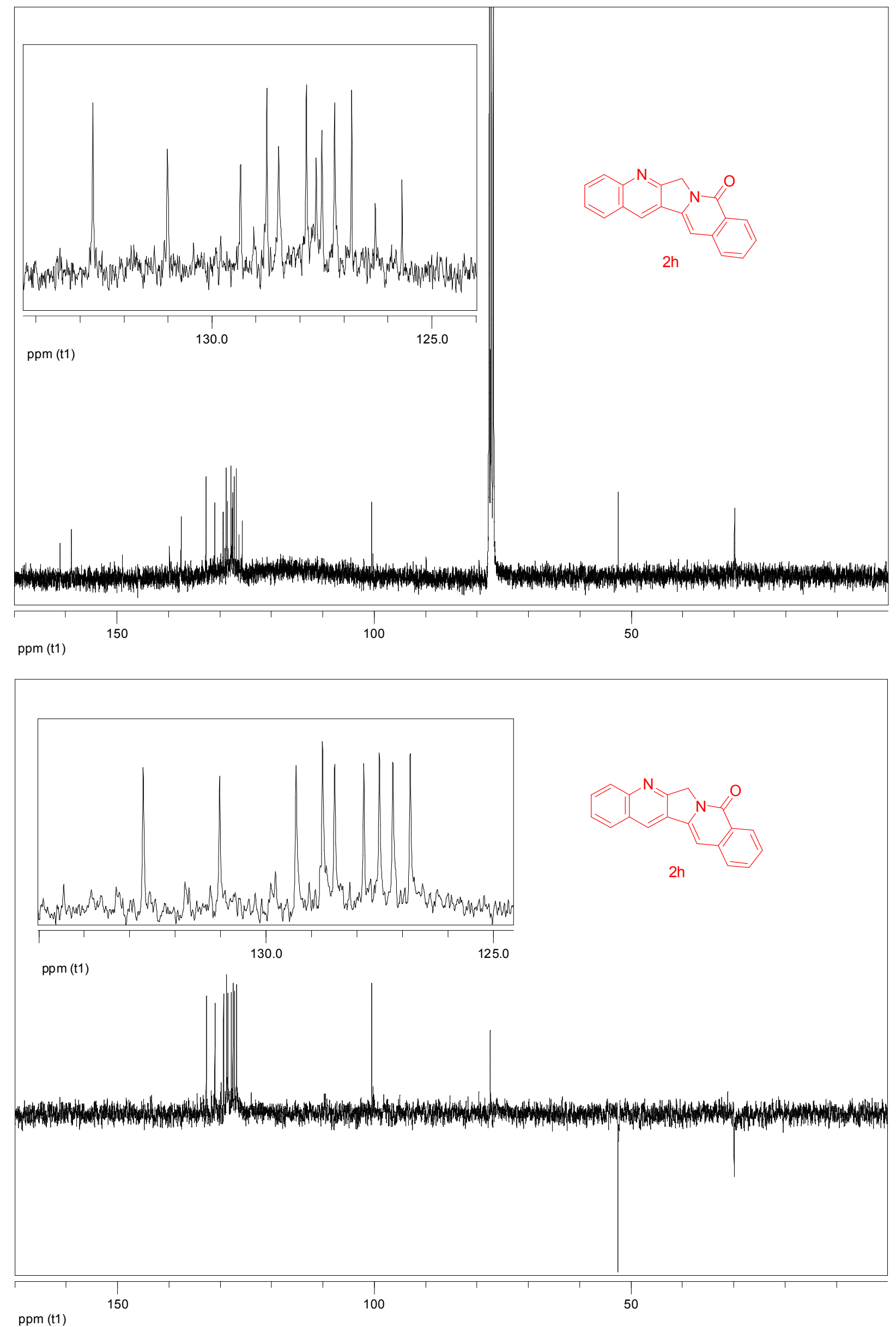

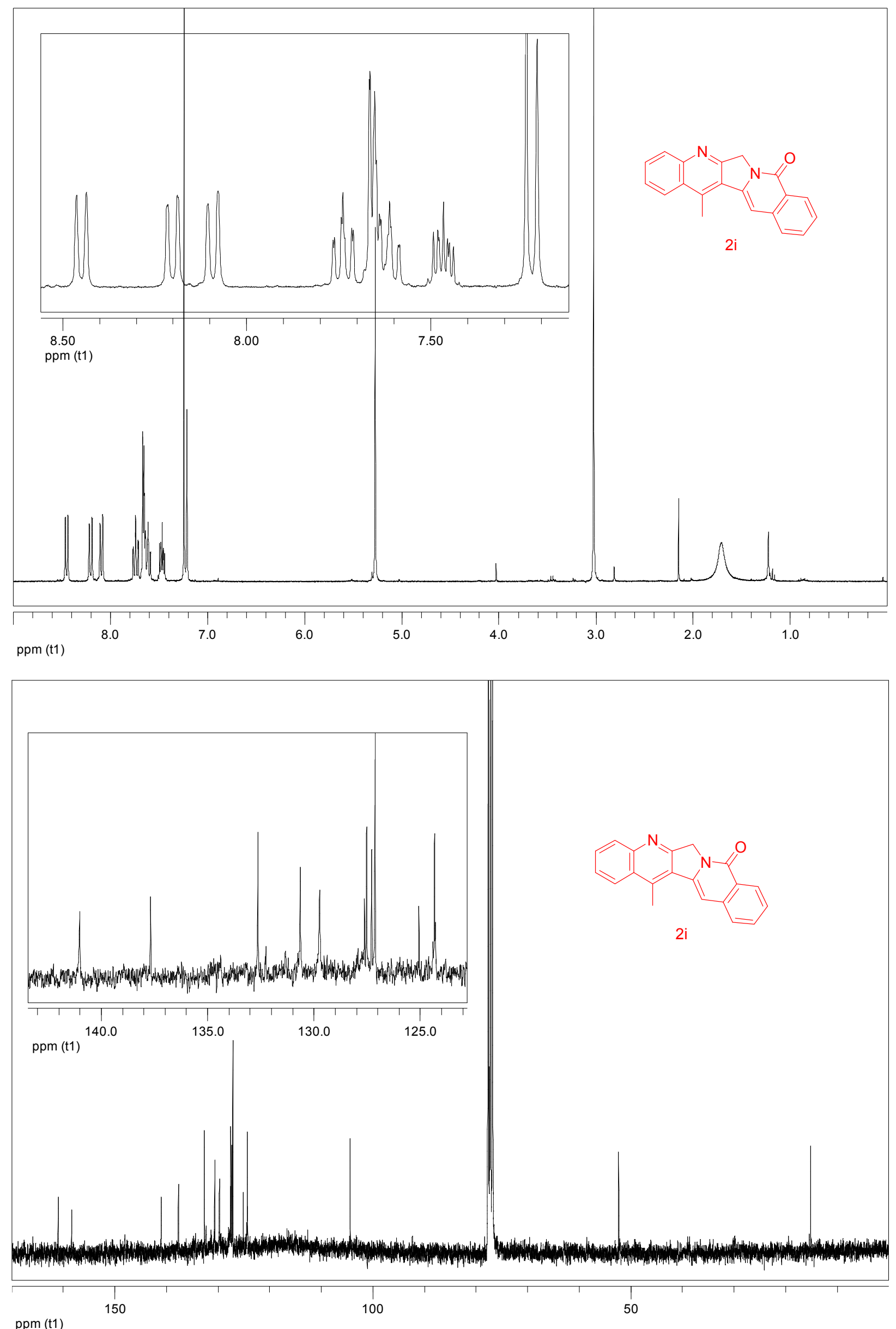

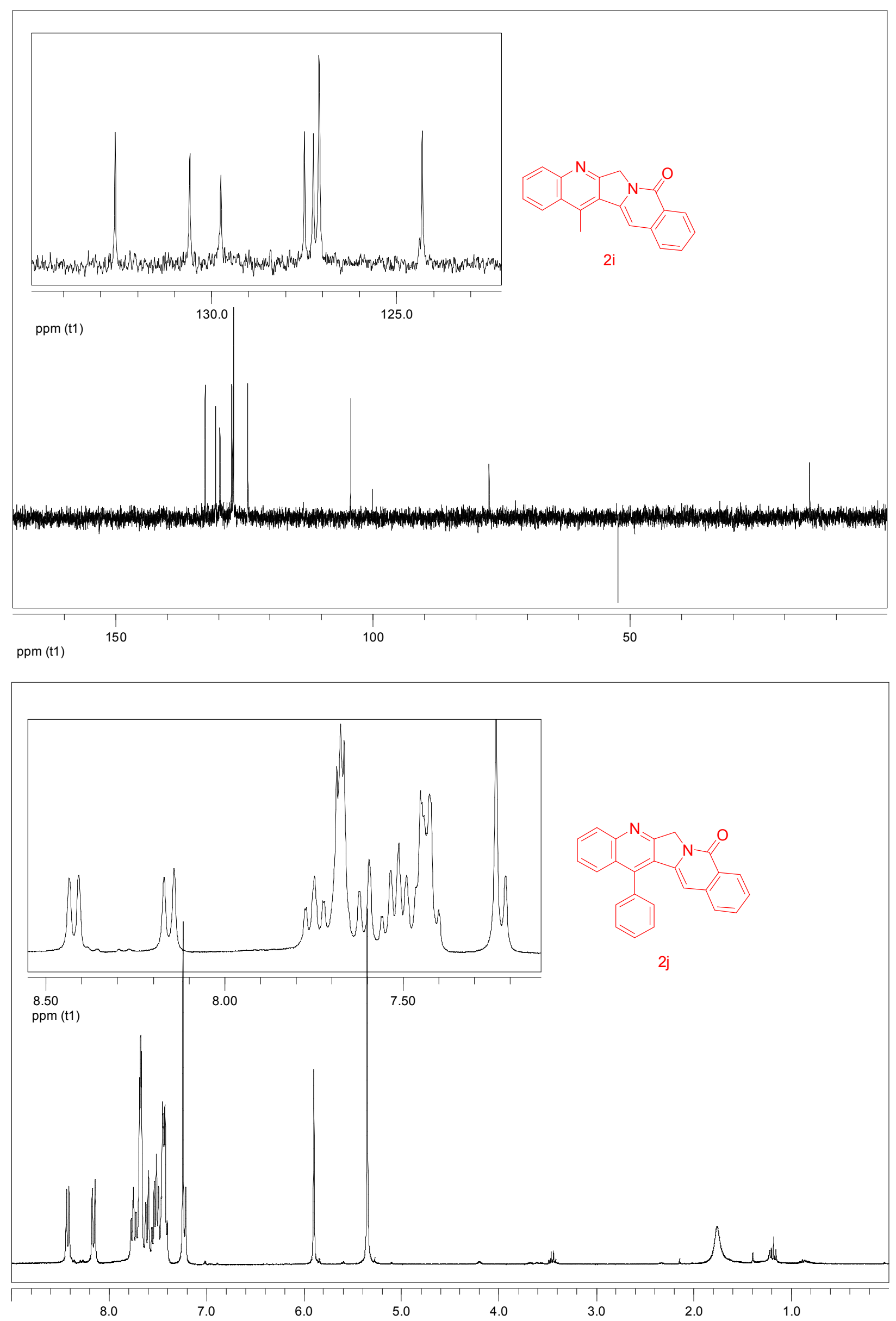

ppm (t1) 


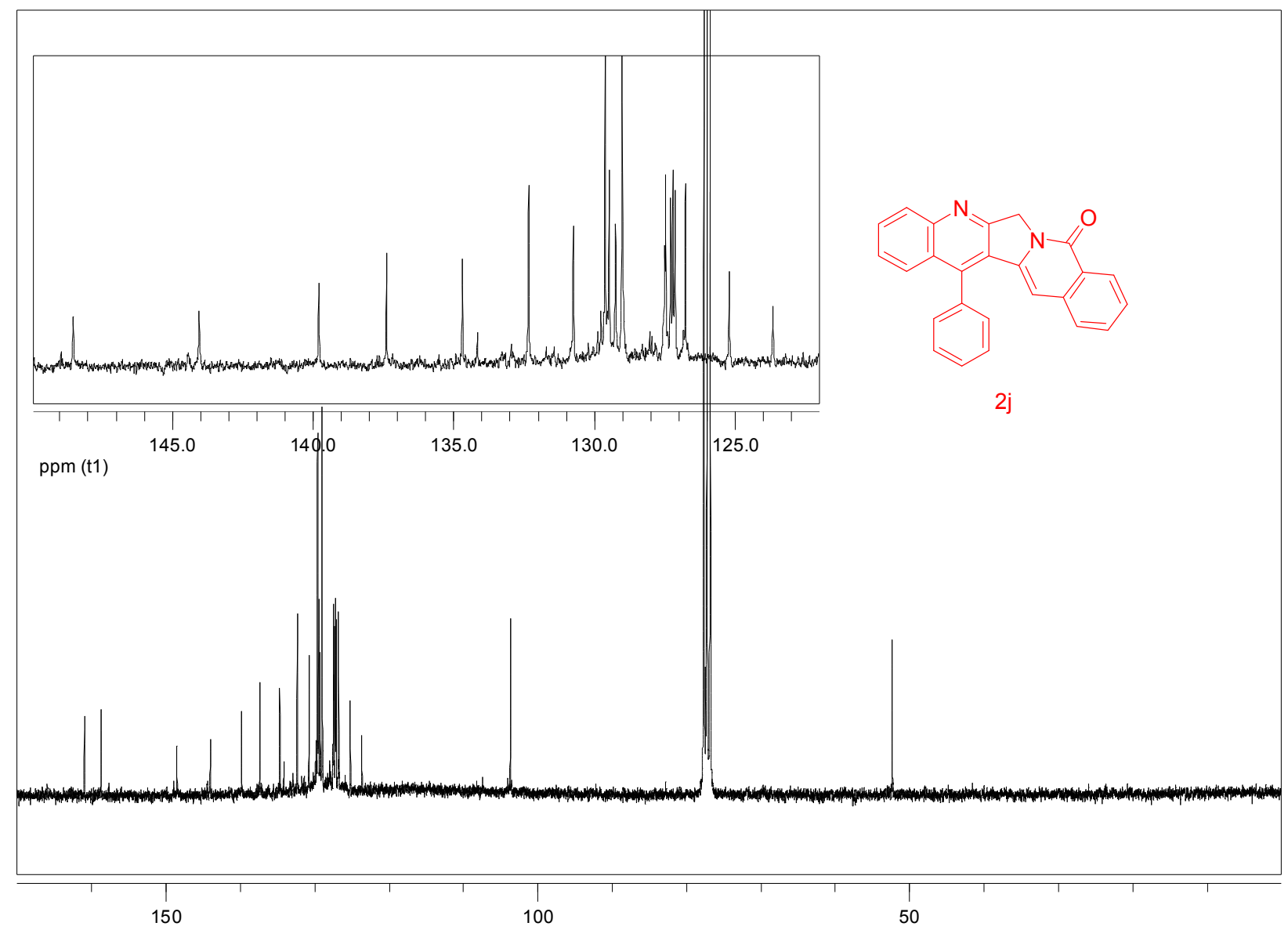

ppm (t1)

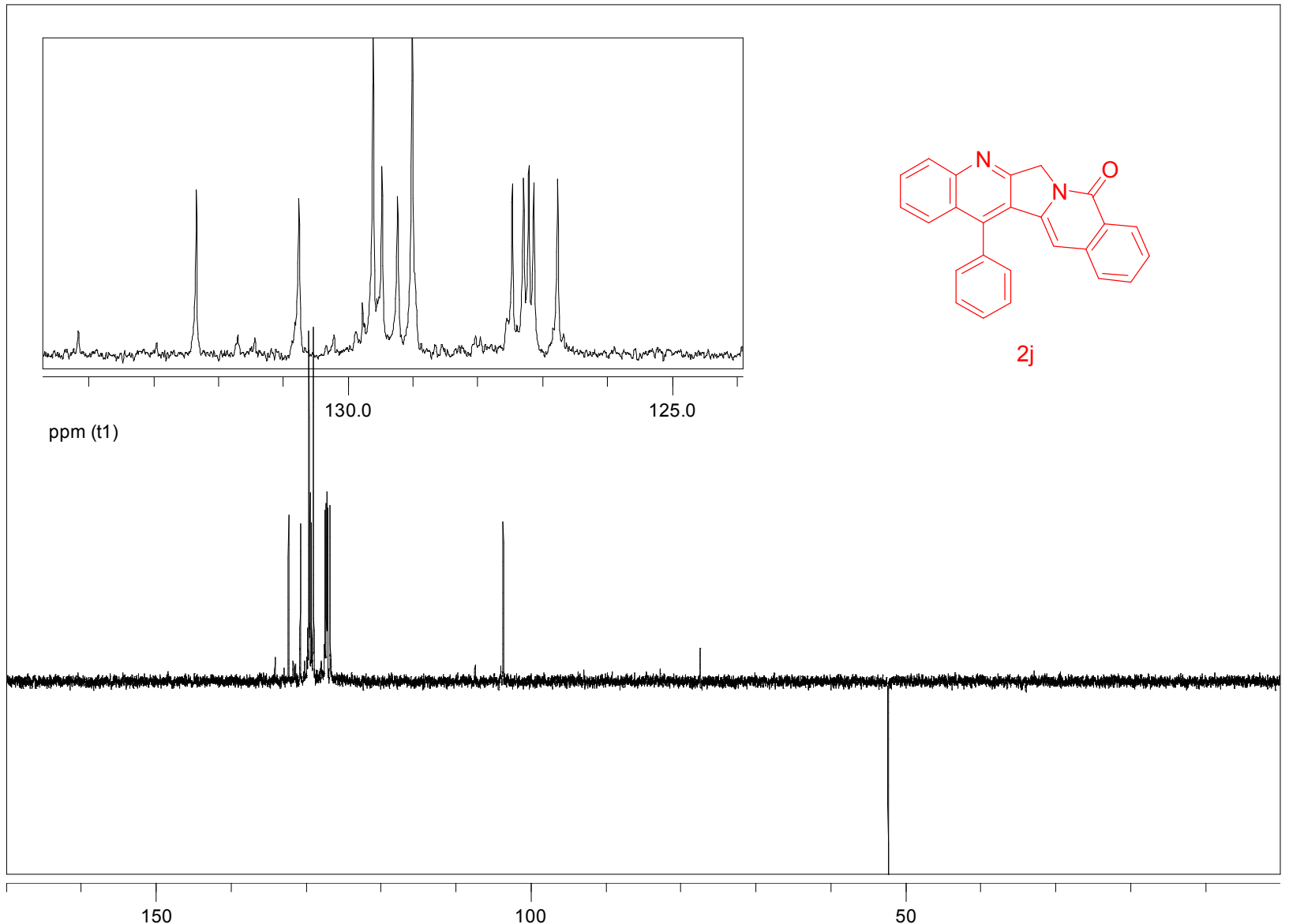

ppm (t1) 


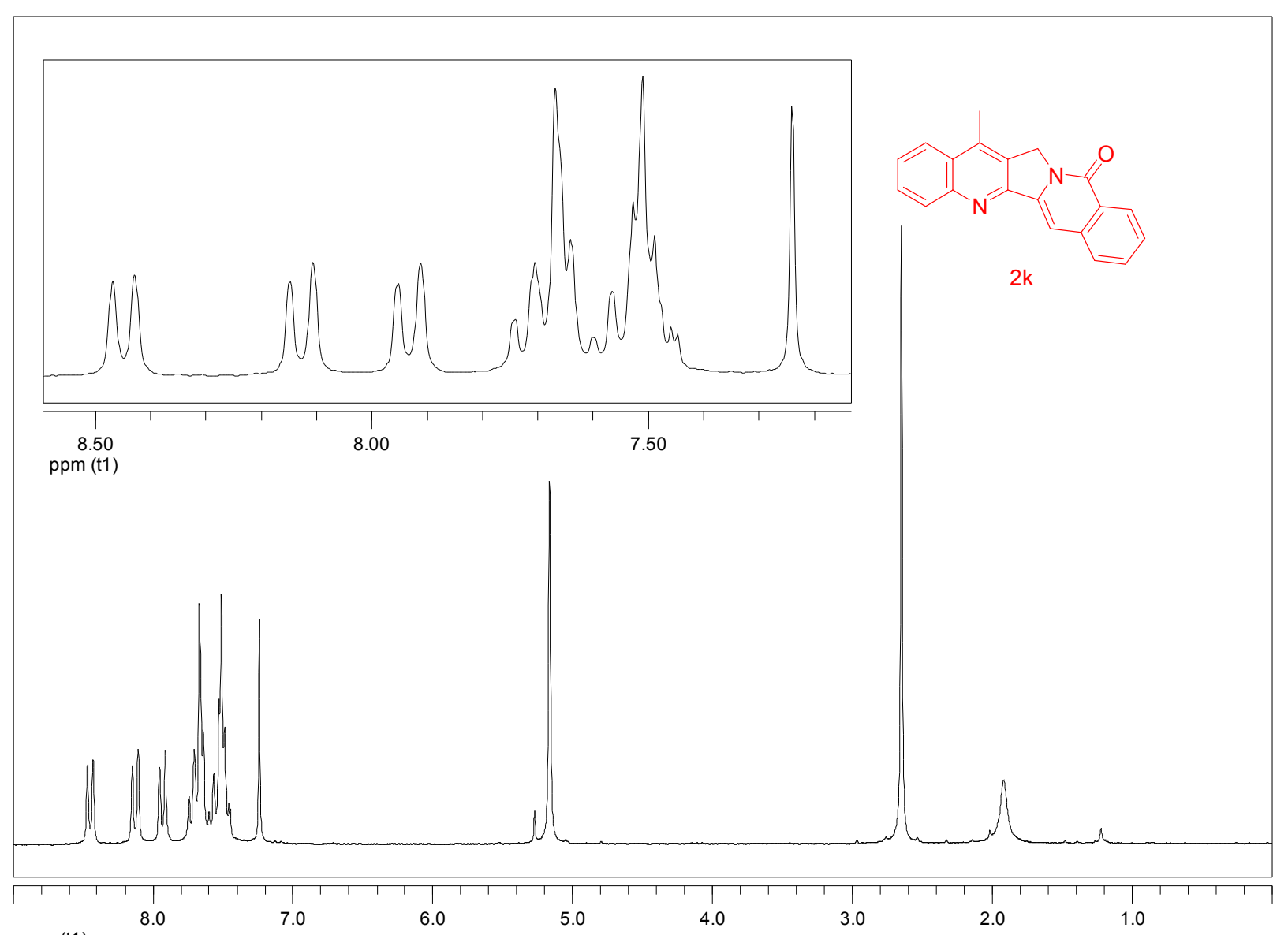

ppm (t1)

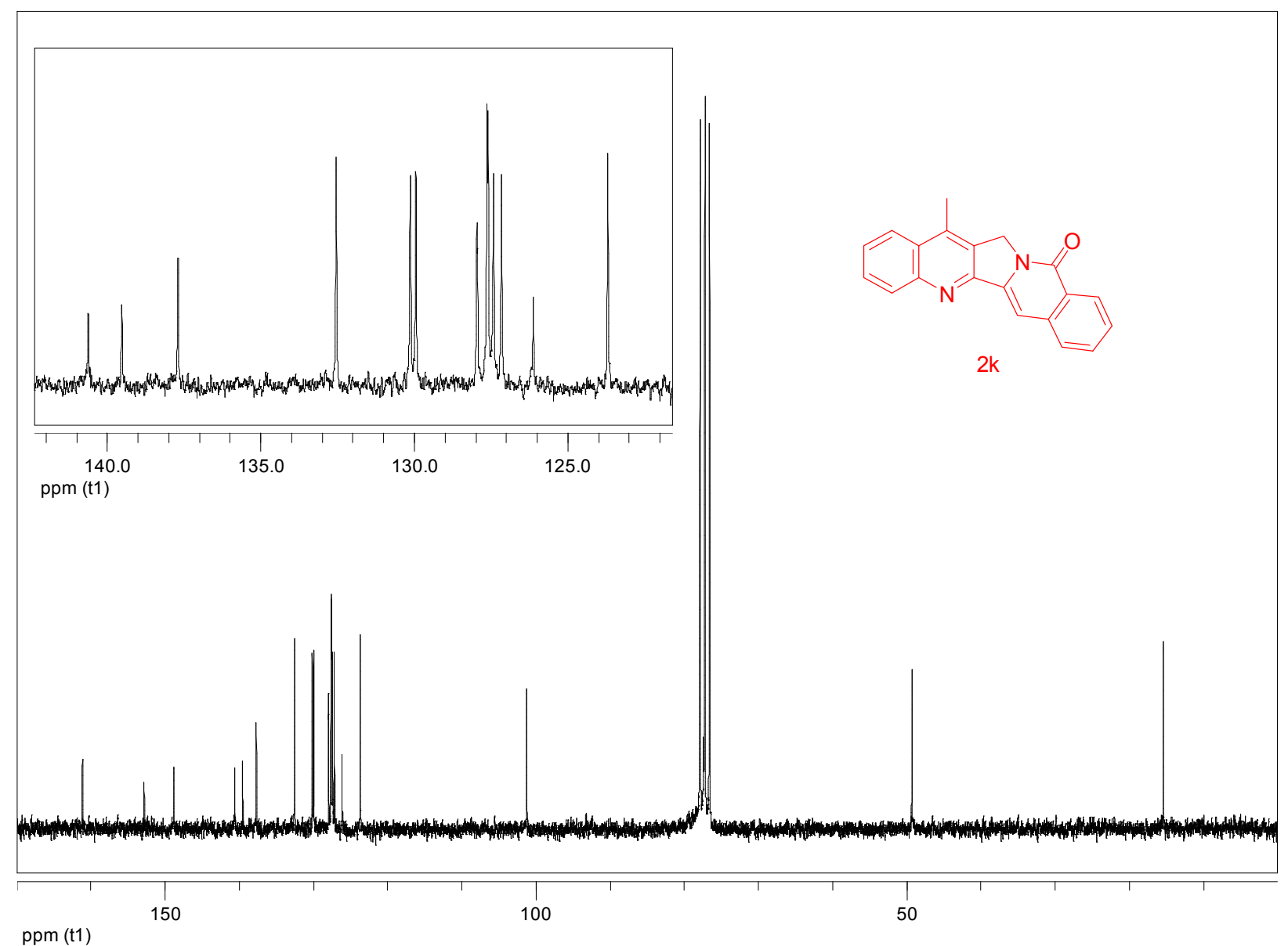




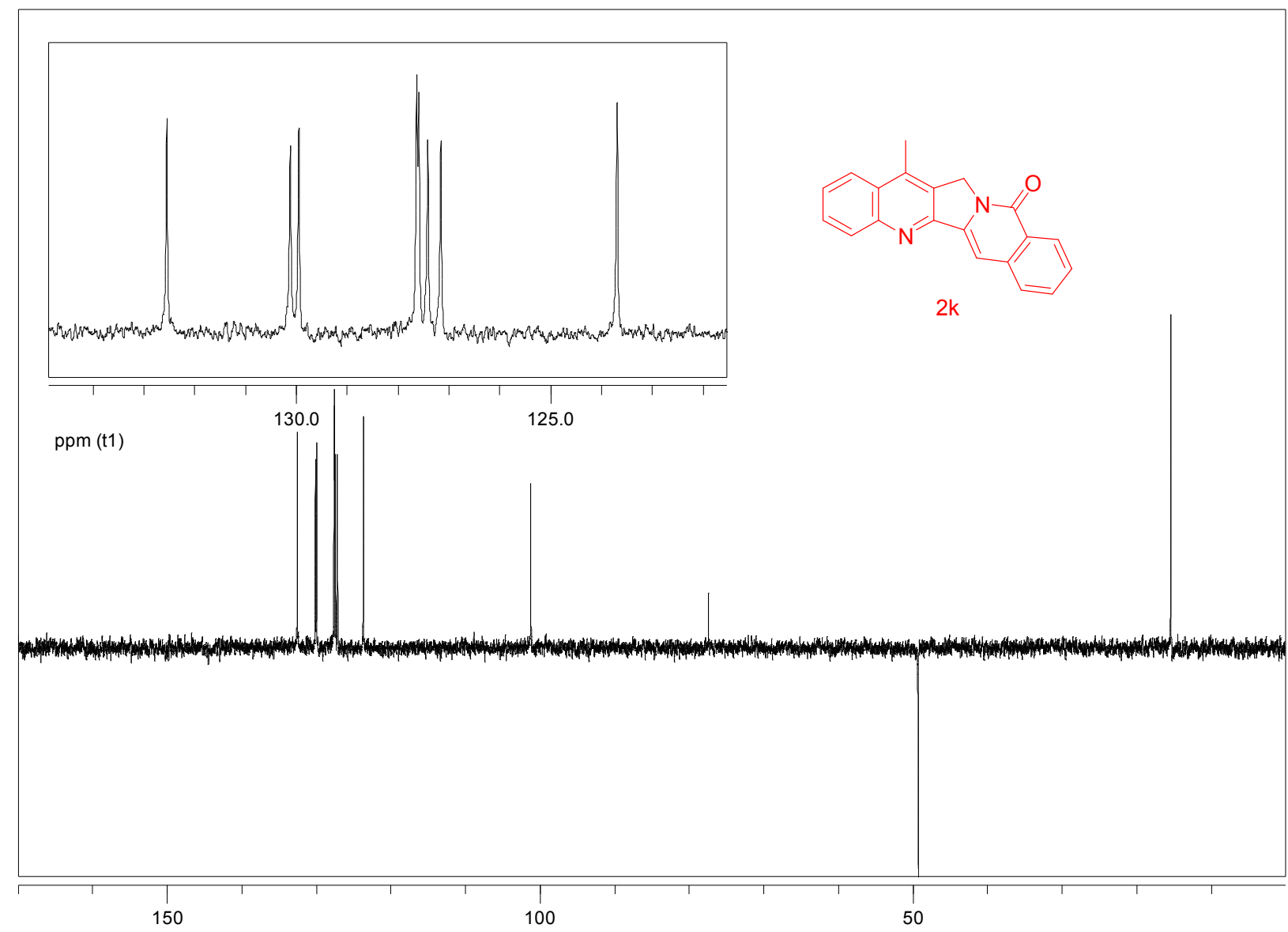

ppm (t1)

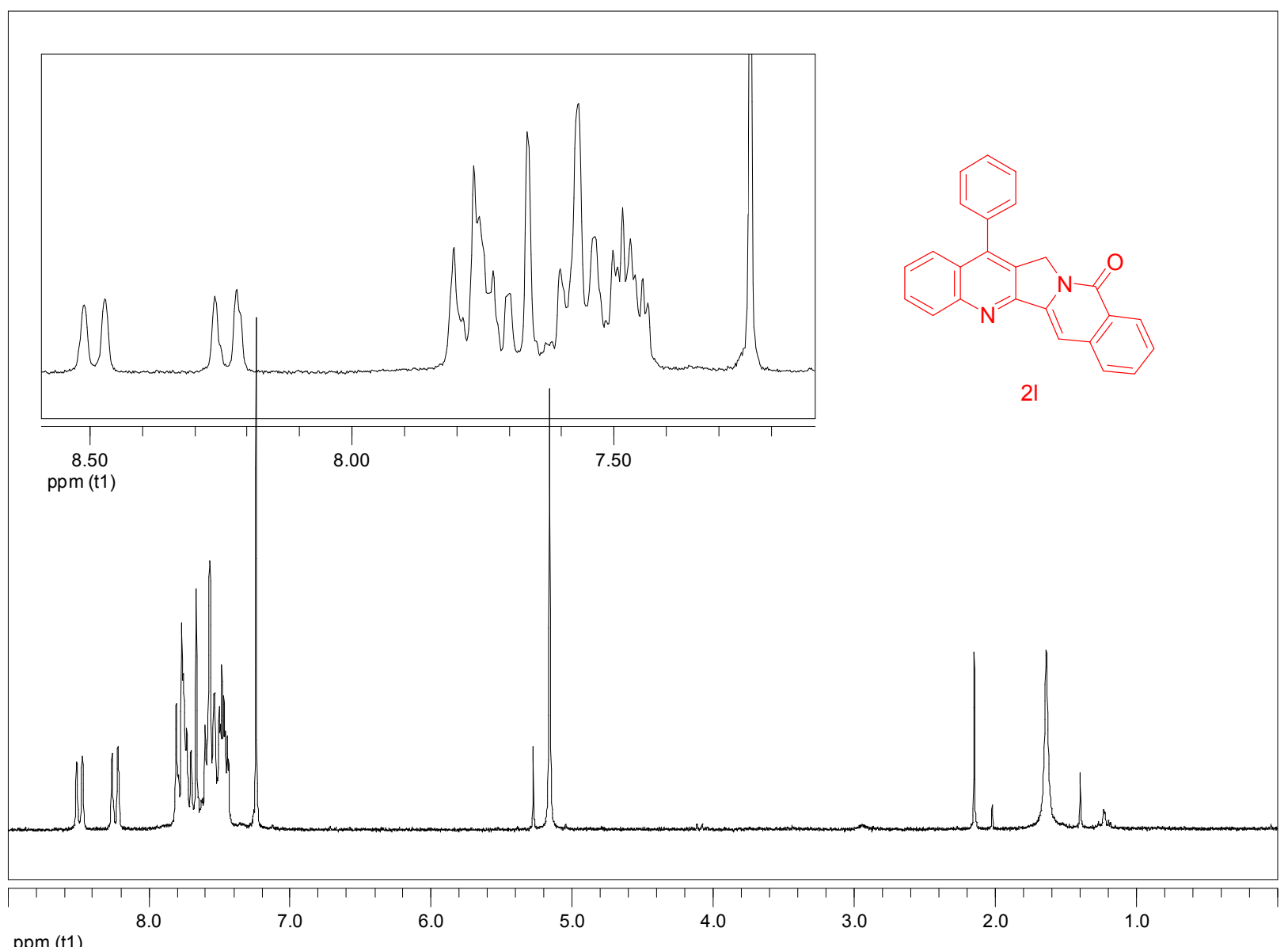

ppm (t1) 

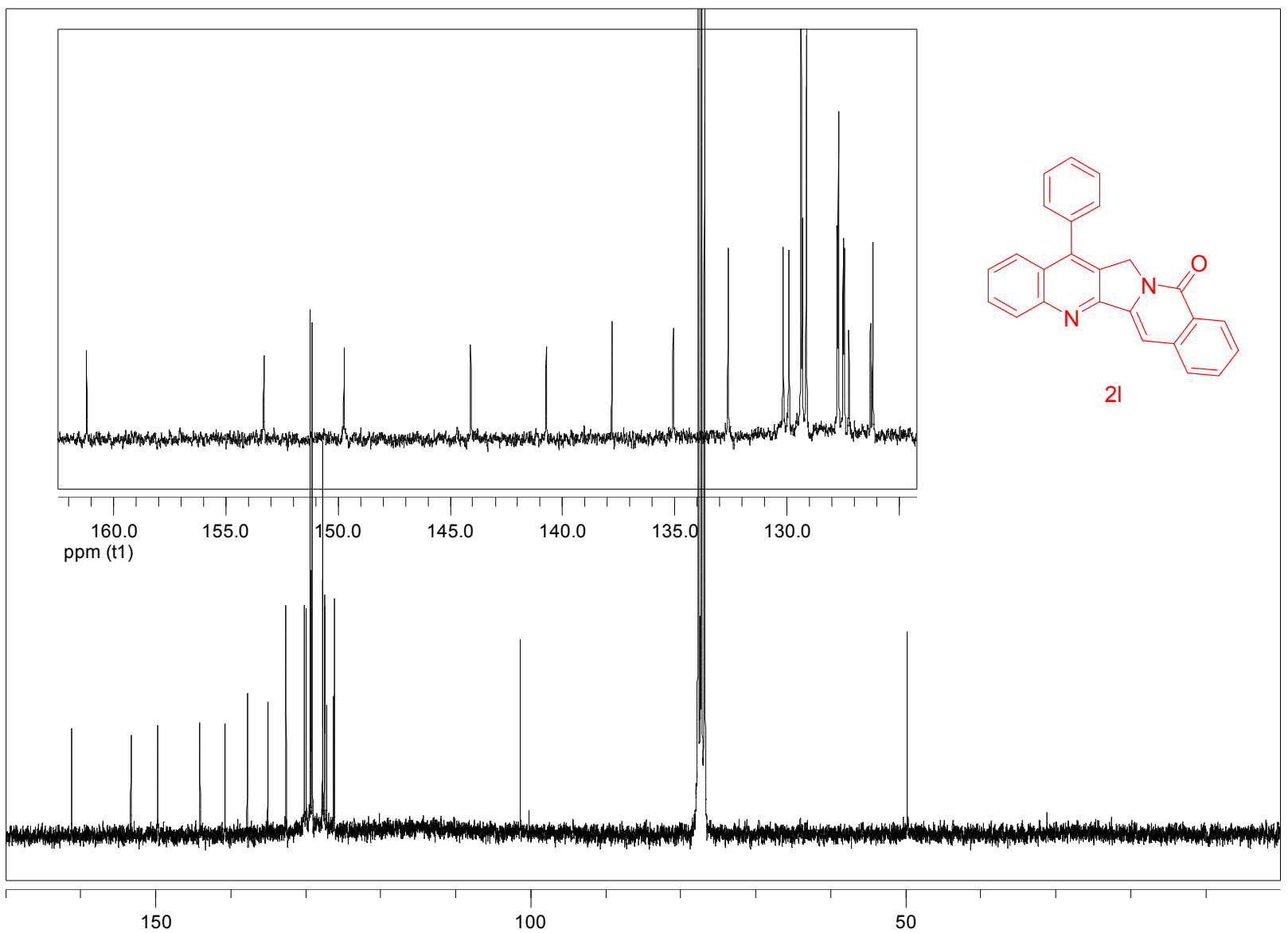
ppm (t1)

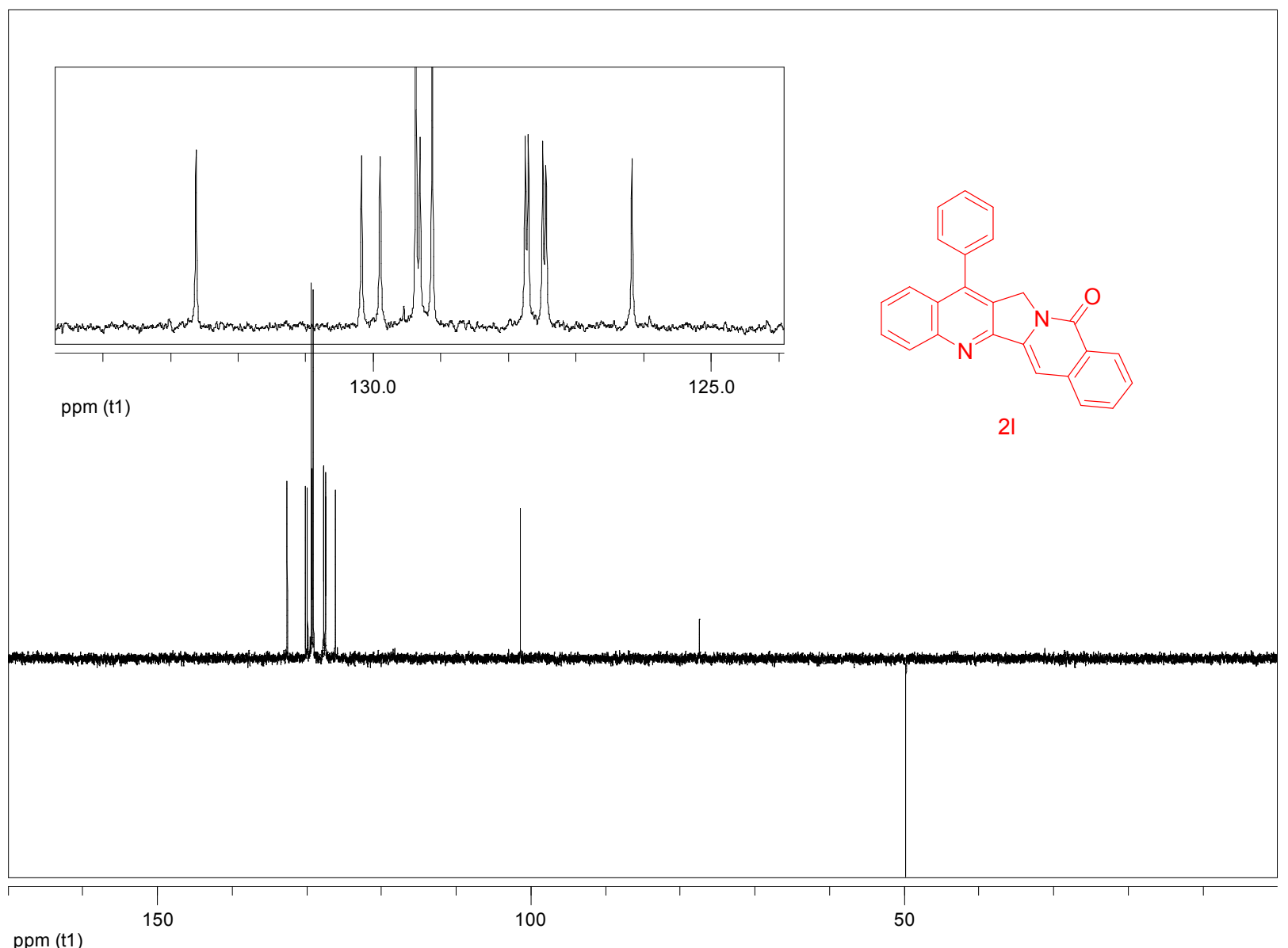

ppm (t1) 